\title{
What Were They Thinking? An Experimental Investigation of Child Sexual Offenders' Beliefs.
}

\author{
By
}

\section{Kirsten Keown}

A thesis submitted to the Victoria University of Wellington in fulfilment of the requirements for the degree of Doctor of Philosophy in Psychology

January 2008

The research studies in this thesis have been supported by the New Zealand Department of Corrections 


\section{Declaration}

I hereby declare that this thesis has not been submitted, either in the same or different form, to this or any other university for a degree:

Signature: 


\section{Publications}

Data and literature from this thesis have been reported in the following formats and published or submitted for publication:

Keown, K., Gannon, T.A., \& Ward, T. (in press). What Were They Thinking? An Exploration of Child Sexual Offenders' Beliefs Using a Lexical Decision Task. Psychology, Crime \& Law.

Keown, K., Gannon, T.A., \& Ward, T. (in press). The Effects of Visual Priming on Information Processing in Child Sexual Offenders. Journal of Sexual Aggression.

Keown, K., Gannon, T.A., \& Ward, T. (in press). What's in a Measure? A Multimethod Study of Child Sexual Offenders' Beliefs. Psychology, Crime \& Law. 


\section{Acknowledgements}

First things first, I must thank the top-notch supervisors who guided me through the past three years. Tony Ward, it has been an honour to have you oversee this work. Thank you for your enthusiasm, advice, and good-natured support. Tag (A.K.A. Theresa Gannon), you have always gone above and beyond the call of duty as a secondary supervisor and I can't thank you enough for it. I have learnt so much under your tutelage, despite the fact that you were working half a world away for much of the time. Tony and Tag, I feel proud to have had you as my supervisors and I will miss working with both of you.

Devon Polaschek, your efforts also played a role in the development of this $\mathrm{PhD}$. From assisting with grant funding, to reviewing my work, to providing advice, you were always generous with your time. Than you for allowing me to tap the wealth of knowledge that resides between your ears.

Many thanks to Marc Wilson and Nokuthaba Sibanda for statistical support. I'm not sure where people like me would be without the expertise and generosity of people like you, but I'm sure a lot more hair-pulling and cursing of statistical manuals would be involved.

I am grateful also to the New Zealand Corrections staff who supported this research and took time out from their busy schedules to help me. And of course, many thanks to the participants who made this research possible. I asked many of you to do things that you found challenging and I am grateful for, and admire, the efforts you made.

Special thanks to my dear, lifelong friend Corrine. Sweetie, I'm so grateful for your encouragement and unwavering belief in my ability to get this done. And of course you were right all along; no matter how tough the going, in the end things do fall into place. While I'm on the subject of friends, thank you to the other special women in my life 
who encouraged me and didn't criticise (not openly, anyway!) my virtual disappearance these past three years. I'm talking about you, Eva, Jo, Verity, Marisa, and Kate.

I also want to thank my family, who kept me largely sane throughout the past three years. Like any good family you simultaneously kept me laughing at, and believing in, myself. Thank you especially to my parents for always encouraging and taking pride in my academic pursuits.

Above all, I must express my gratitude to Paddy - the man who really deserves an acknowledgments section all of his own. Profoundest thanks to you, my dear, brilliant, incomparable husband. Without your patience, advice, and boundless support this thesis could not have happened. One of these days I should make you breakfast in bed or something. 


\title{
Conventions Used in this Thesis
}

\section{Numbering Studies}

The three studies in this thesis are numbered independently of the chapter in which they appear.

\section{Numbering Tables and Figures}

All tables and figures are numbered according to the chapter in which they appear. They are numbered as figure or table $x, y$., with $x$ referring to the chapter number and $y$ referring to the order in with the figure or table appears within that chapter.

\author{
Abbreviations \\ refer to the following list:

$\begin{array}{ll}\text { CSO } & \text { Child Sexual Offender } \\ \text { OC } & \text { Offender Control } \\ \text { CC } & \text { Community Control } \\ \text { IT } & \text { Implicit Theory } \\ \text { RT } & \text { Reaction Time } \\ \text { IAT } & \text { Implicit Association Task } \\ \text { LDT } & \text { Lexical Decision Task } \\ \text { RSVP-M } & \text { Rapid Serial Visual Presentation (Modified) } \\ \text { ms } & \text { Milliseconds }\end{array}$

Although abbreviations have been described in the text the reader may find it helpful to 


\begin{abstract}
In the field of forensic psychology, child sexual offenders (CSOs) are often hypothesised to hold abnormal beliefs that facilitate the onset and maintenance of their offending. This idea has had considerable impact upon current CSO assessment and treatment practices. However, despite its intuitive appeal, empirical evidence supporting the hypothesis is unfortunately lacking.

Information regarding the role that cognition plays in child sexual offending has been gathered almost exclusively using self report (i.e., interview and questionnaire) methods. In interview studies, CSOs talk at length about their offending and their statements are analysed for the presence of so-called cognitive distortions: utterances deemed to represent abnormal, offence-facilitating beliefs. In questionnaire studies CSOs and controls rate the veracity of listed cognitive distortion items and their answers are compared. In general, interview and questionnaire studies have tended to find that CSOs endorse cognitive distortions, which seemingly supports the notion that they hold offencesupportive beliefs. However, serious issues plague the use of these self-report methods because endorsement of cognitive distortions might reflect phenomena other than beliefs.
\end{abstract}

The primary aim of this thesis was to examine the idea that CSOs hold offencesupportive beliefs using methods designed to side-step issues associated with self-report methods. Across three studies, three cognitive experimental techniques were for the first time applied to the study of CSO cognition. In Study One, CSOs and offender and community controls completed an experimental procedure called the lexical decision task. Against hypotheses, when compared to controls CSOs did not interpret offence-related sentences in line with distorted beliefs. A possible explanation for this finding was that CSOs' offence-supportive beliefs were insufficiently activated during testing.

To investigate, in Study Two half the CSO and half the offender control participants were primed with images of scantily-clad children before commencing 
experimental testing. During testing, CSOs and offender controls read sentences describing children behaving in potentially sexualised ways. Participants were then given a surprise recognition test in which half the sentences were re-presented in an unambiguously sexual form, and half in an unambiguously nonsexual form. Contrary to hypotheses, neither primed nor control child sexual offenders showed memory biases for sexualised sentences, suggesting they did not interpret the original sentences in line with offence-supportive beliefs.

Finally, in Study Three, CSOs' beliefs were examined using interview methods, and CSOs' and offender controls' beliefs were measured using a questionnaire as well as an experimental technique that used sentence reading times to implicitly measure beliefs. As hypothesised, CSOs showed evidence of holding offence-supportive beliefs according to the interview and questionnaire measures, but against predictions they demonstrated no experimental evidence of such beliefs. In fact, the three methods showed virtually no agreement regarding the belief-types each CSO was deemed to hold, raising important questions about the phenomena measured by each method.

Overall, the results of this thesis did not support the stance that CSOs generally hold offence-supportive beliefs that set them apart from others. The implications of these findings for theory and treatment are discussed and directions for future research are suggested. 


\section{Contents}

Declaration $\quad$ II

$\begin{array}{lll}\text { Publications } & \text { III }\end{array}$

$\begin{array}{lll}\text { Acknowledgements } & \text { IV }\end{array}$

$\begin{array}{ll}\text { Conventions Used in this Thesis } & \text { VI }\end{array}$

Abstract $\quad$ VII

$\begin{array}{lll}\text { Contents } & \text { IX }\end{array}$

$\begin{array}{ll}\text { List of Tables } & \text { XIVI }\end{array}$

List of Figures $\quad$ XV

$\begin{array}{ll}\text { Chapter One } & 1\end{array}$

What Were They Thinking? Current Theorising About Child Sexual

Offenders' Cognitions. $\quad 1$

Introduction 1

Introductory concepts - A framework for cognition 3

$\begin{array}{ll}\text { Cognitive structures } & 3\end{array}$

Cognitive processes $\quad 5$

$\begin{array}{ll}\text { Cognitive products } & 6\end{array}$

Types of Theory Regarding Child Sexual Offending 7

$\begin{array}{ll}\text { Level I (multifactorial) theories } & 7\end{array}$

Level II (single-factor) theories $\quad 8$

Level III (microtheory) theories $\quad 8$

Multifactor Theories of Child Sexual Offending 8

Finkelhor's (1984) Pre-conditions Model 9 
Cognitive Distortions as Beliefs

What's in a Measure? Research Underpinning the Notion of Offence-Supportive Beliefs

Interview Studies of CSO Statements

Questionnaire Studies of CSO Statements

A New Paradigm: Information Processing Studies

What's in a Measure?

A general note on the recruitment of participants

A general note on written material provided to participants

\section{Chapter Four}

\section{Decision Task}

Introduction 
$\begin{array}{ll}\text { Design } & 73\end{array}$

$\begin{array}{ll}\text { Materials } & 73\end{array}$

$\begin{array}{ll}\text { Apparatus } & 75\end{array}$

$\begin{array}{ll}\text { Procedure } & 75\end{array}$

$\begin{array}{ll}\text { RESULTS } & 77\end{array}$

$\begin{array}{ll}\text { Data preparation } & 77\end{array}$

Main analysis of response times $\quad 80$

$\begin{array}{ll}\text { DISCUSSION } & 84\end{array}$

$\begin{array}{ll}\text { Chapter Five } & 91\end{array}$

The Effects of Visual Priming on Information Processing in Child

$\begin{array}{ll}\text { Sexual Offenders } & 91\end{array}$

$\begin{array}{ll}\text { Introduction } & 91\end{array}$

Study Two: The Effects of Visual Priming on Interpretation of Ambiguous Sentences Related to Child Behaviour 92

$\begin{array}{ll}\text { METHOD } & 94\end{array}$

$\begin{array}{ll}\text { Participants } & 94\end{array}$

$\begin{array}{ll}\text { Design } & 98\end{array}$

$\begin{array}{lr}\text { Materials } & 99\end{array}$

$\begin{array}{ll}\text { Apparatus } & 101\end{array}$

$\begin{array}{ll}\text { Procedure } & 102\end{array}$

$\begin{array}{ll}\text { RESULTS } & 103\end{array}$

Data Preparation: Reaction Times 103

Data Preparation: Recognition Analysis 104

Main analysis of picture viewing times 105

Main analysis of sentence recognition 105 
A Multimethod Study of CSOs' Offence-Supportive Beliefs.

Beliefs

METHOD

Participants

Overall Design

Measures

Procedure

RESULTS

Questionnaire Analysis

Agreement Across Measures

DISCUSSION

Chapter Seven

What Were They Thinking? General Conclusions 145

$\begin{array}{ll}\text { Introduction } & 145\end{array}$

Lexical Decision Task 146

$\begin{array}{ll}\text { Primed Memory Study } & 147\end{array}$

$\begin{array}{ll}\text { The Multimethod Study } & 148\end{array}$

$\begin{array}{lr}\text { Limitations } & 150\end{array}$

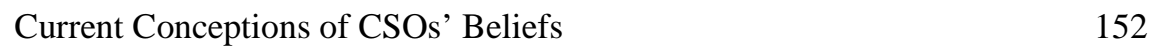


Prevalence of CSOs' Offence- Supportive Beliefs

The Nature of Offence- Supportive Beliefs

Research Implications

Treatment Implications

Final Summary

References 


\section{List of Tables}

\section{Table 2.1.}

Cognitive distortions categories noted in Abel, Becker, and Cunningham-Rathner

\section{Table 2.2.}

Ten most common cognitive distortions categories and percentage of offenders producing

response, as per Neidigh and Krop (1992)

Table 4.1.

Demographic details for participant groups: Study 1

Table 4.2.

Participant groups' mean reaction times (in milliseconds) to LDT words and nonwords. 79

\section{Table 5.1.}

Demographic details for participant groups: Study Two

Table 6.1.

Demographic details for participant groups: Study Three

Table 6.2.

Classification of IT endorsement, per individual, according to three methods

Table 6.3.

Percentages of CSOs classed as holding three belief clusters by measure $(n=33)$

Table 6.4. 


\section{List of Figures}

\section{Figure 4.1.}

Mean Differences (+/- SEM) in Offence-supportive and Nonoffence-Supportive Reaction

Times to All Implicit Theories

\section{Figure 4.2.}

Mean Differences (+/- SEM) in Offence-supportive and Nonoffence-Supportive Reaction

Times to Uncontrollable

Figure 5.1.

Study Two Experimental Design

Figure 5.2.

Mean Recognition Differences (+/- SEM) for Sexual Recognition

Figure 5.3.

Mean Reaction Time Differences (+/- SEM) in Milliseconds (ms) for Sexual

Figure 6.1.

Study Three Research Design Overview

Figure 6.2.

RSVP-M Design 


\section{Chapter One}

\section{What Were They Thinking? Current Theorising About Child Sexual Offenders' Cognitions.}

\section{Introduction}

The serious social costs that attend child sexual offending are well-documented. Its victims typically experience various adverse, long-term effects such as depression, anxiety, low self-esteem, self-destructive behaviour and intimacy difficulties (Browne \& Finkelhor, 1986; Denov, 2004; Mullen, Martin, Anderson, Romans, \& Herbison, 1996). Considerable financial resources are required to alleviate these ill-effects and to incarcerate the perpetrators of child sexual abuse (Chaffin, 1994; Fromm 2001; Purvis \& Joyce, 2005).

Despite these adverse consequences and the high levels of public condemnation that child sexual abuse attracts, its incidence is far from uncommon. While prevalence estimates in western countries vary widely across studies, it appears that around $20 \%$ to $30 \%$ of females and $10 \%$ to $15 \%$ of males have experienced child sexual abuse (Briere, 1992). In New Zealand, where The Crimes Act (1961) places the legal age of consent at 16 years of age, findings from a large longitudinal study suggested that $18.5 \%$ of people under the age of 16 are sexually abused by adults (Fergusson, Horwood, \& Woodward, 2000).

Why do certain individuals become child sexual offenders (CSOs)? What drives them to pursue an activity that is illegal, harmful to others, and largely reviled by the general public? This intriguing question has captured the attention of a large body of psychological researchers and theoreticians, and various factors thought to play an etiological role in child sexual offending have been highlighted. These range from broad sociological factors such as the sexualised portrayals of young people that occur in the 
media (Stermac, Segal, \& Gillis, 1990), to individually-situated, psychological mechanisms such as low self esteem (Marshall, Cripps, Anderson, \& Cortoni, 1999a) and emotional regulation problems (Marshall, 1999). As will be discussed in the following pages, theories of child sexual offending have over time assigned increasing importance to the role of individual, psychological factors, with particular attention being paid to the role of cognition in sexual offending. Consequently, many researchers and clinicians accept the view that most or all CSOs hold stable, entrenched beliefs that facilitate their offending and set them apart from other non-offending individuals. This view certainly has intuitive appeal; after all, how could one commit such publicly denounced crimes without holding exceptional beliefs that somehow make such offending seem acceptable? This thesis will investigate what, if any, empirical evidence there is to support this intuition.

To set the scene, this chapter will begin with a brief introduction to the information processing framework; a view of human mental activity that has been particularly influential in cognitive psychology and in the study of CSOs. Chapter content will then turn to a review of four influential theories that seek to explain how multiple factors interact to elicit sexual offending. The review will focus on the types of cognitions that each model highlights and the etiological role those cognitions are thought to play. It will be shown that there is disagreement over the definitions and mechanisms hypothesised as being associated with offence-supportive cognitions, both within and across the four models.

Chapter Two will turn to focus on specific single-factor theories of CSOs' offencesupportive cognitions. It will be shown that although confusion regarding the nature and role of CSOs' offence-supportive cognitions also permeates this area of the literature, there is nevertheless a sense that the key cognitions of interest are faulty, offencefacilitating beliefs that set CSOs apart from others. Chapter Three explores what, if any, evidence exists that CSOs do in fact hold offence-supportive beliefs. Issues plaguing the 
measurement of these beliefs will be outlined. Chapters Four, Five, and Six will present three unique experimental studies that aim to test the idea that CSOs have offencesupportive cognitions that are held in belief- or schema-like structures. These studies were all designed to overcome problems inherent in the use of traditional measures. Importantly, the last of these studies will also triangulate findings regarding these beliefs as measured by an implicit, cognitive-experimental method and two explicit, conventional methods (i.e., interview and questionnaire). Finally, in Chapter Seven the thesis will conclude with a general discussion that summarises and integrates overall findings.

\section{Introductory concepts - A framework for cognition}

Researchers studying the cognitions of CSOs have discussed and labelled a huge range of cognitions thought to play a role in commissioning sexual offending behaviour. To get to grips with this plethora of terms it will be helpful to first consider a meaningful system for classifying components of cognition and understanding their interrelations. While there are many ways of conceptualising the human cognitive architecture and its associated processes and functions, the information processing framework has been particularly influential. Under the information processing framework, human cognition is viewed as being computational in nature. That is, data from the environment is thought to be represented in the mind in the form of symbols, upon which operations are performed (Varela, Thompson, \& Rosch, 1991). The three key components of this information processing system have been identified by Hollon and Kris (1984) as cognitive structures, cognitive processes, and cognitive products. Each component is described below.

\section{Cognitive structures}

The human mind can be conceptualised as a vast network of information nodes that describe features of objects or events. As individuals process environmental stimuli, specific information nodes are activated, (i.e., brought into working memory). When two or more information units are repeatedly activated together they become linked, such that 
activating one unit can automatically activate the other (Collins \& Loftus, 1975). These linked bodies of information are called cognitive structures. In other words, cognitive structures are organised clusters of interrelated memories that form in response to life experiences (Collins \& Loftus, 1975).

An important property of cognitive structures is that when one unit in the structure is activated, closely associated memories and information units also activate (Collins \& Loftus, 1975, Hollon \& Kris, 1984). So, for instance, reading the word "purr" can activate information about cats. This information could include semantic knowledge about cats (e.g., they are four-legged animals) and episodic memories of cats (e.g., mental images of cats). Importantly, the co-activation of cognitive structures influences the way humans perceive, predict, interpret, and understand the world (Fiske \& Taylor, 1991; Kilhstrom \& Klein, 1994; Ward, Hudson, Johnston, \& Marshall, 1997). Rather than processing incoming stimuli anew each time we encounter an event, humans utilise stored information to observe and evaluate that event (e.g., Bartlett, 1932; Dodge \& Frame, 1982). Consequently, cognitive structures can bias information gathering and storage such that one only sees and remembers information that aligns with existing memory stores. Take, for instance, a woman who holds a schema that frames academics as dull. Upon meeting an academic, this (clearly misguided!) woman will be more likely to interpret the academic's words and actions as signals of dullness. In addition, she will notice and remember information that confirms her view of the academic, while discarding evidence to the contrary.

Generally, cognitive structures are referred to as schemas (Hollon \& Kris, 1984), although other words such as attitude and belief are sometimes used to denote cognitive structures (Schroder, Driver, \& Streufert, 1967; Ward, 2000). In fact, the demarcations between schemas, beliefs, and attitudes are ill-defined, with the words often being used interchangeably (e.g., Augoustinos \& Walker 1995; Fiske \& Taylor 1991; Freeman, 
Pretzer, Fleming, Simon, 2004; McGinn \& Young, 1996; Malamuth, Heavey \& Linz, 1993).

In this thesis both schemas and beliefs will be referred to frequently, hence it is important to delineate the relationship between these two cognitive structures. Beliefs are propositions whose content is held by the individual to be true (Nisbett \& Ross, 1980). Like schemas, they are configurations of stored information that can engender expectancies and provide frameworks for interpreting new experiences. In fact, schemas are generally conceptualized as structures that contain interrelated beliefs (Sherman, Judd, \& Park, 1989; Ward, 2000). However, schemas tend to be more extensive than beliefs (Nisbett \& Ross, 1980) and can contain additional information such as affective "tags" that allow one to evaluate objects as good or bad (Bargh \& Chartrand, 1999; Fiske \& Linville, 1980). Nevertheless, both beliefs and schemas are cognitive structures that hold cognitive content (i.e., information) and that organise the processing and storage of information.

\section{Cognitive processes}

Cognitive processes refer to the ways in which cognitive structures (and their contents) are used to process incoming information (Hollon \& Kris, 1984). Such processes include where our attention is directed, how we encode, interpret and retrieve information, and how we predict what will happen next. To quote Hollon and Kris (1984), they are the "transformational rules for turning input into judgements" (p. 40). An important point to note about cognitive processes is that all humans commit processing errors. All humans engage in cognitive processing that is biased by the content held in their cognitive structures. For instance, when individuals process the world around them their attention is guided by what they believe is important and what they expect to see, which can lead them to ignore information that is relevant to the assessment of an event or object (Fiske \& Taylor, 1991). Thus, as Hollon and Kris explain, cognitive processes serve to maintain the 
rigidity and constancy of certain cognitive structures. As will be discussed in Chapter Three, information processing errors are sometimes called cognitive distortions (Beck, 1963; 1967), although within the CSO literature cognitive distortions have also been conceived of as products that reflect underlying cognitive structures.

It should be noted that cognitive processes can be influenced by contextual and environmental factors (Fiske \& Taylor, 1991). For instance, contextual factors that increase cognitive load can encourage cognitive "short-cuts", wherein the individual tends to leap to conclusions rather than carefully analyse available information. Similarly, environmental factors that strongly activate schemas will tend to produce schema-driven, rather than data-driven, processing.

\section{Cognitive products}

As cognitive structures and processes interact they give rise to thoughts, attributions, ideas, and decisions. These emergent phenomena are called cognitive products (Hollon \& Kriss, 1984). Cognitive products are accessible cognitions that can be brought to conscious awareness and directly reported. Hollon and Kriss describe cognitive products as metaphorical iceberg tips that offer glimpses into individuals' cognitive structures, content, and processes.

Interestingly, beliefs have been referred to as cognitive products (e.g., Beech \& Mann, 2002; Ward, Fon, Hudson, \& McCormack, 1998) even though, as noted earlier, they are also frequently described as if they are cognitive structures. How is it that beliefs can be viewed as both structures and products? The confusion may arise because beliefs can simply be defined as propositions that individuals assess as true. This definition fails to delineate the underlying processes that drive individuals' assessments, or state whether the assessments necessarily remain static across time (Szentagotai et al., 2005). The word belief is therefore used to denote both stable convictions that consistently influence behaviour (e.g., a belief in God) and temporary products of information processing that are 
driven by transitory situational factors (e.g., the wife who, in the midst of a heated argument, "believes" her husband to be an idiot). To avoid this confusion, in the current thesis the word belief will be used to denote stable cognitions that consistently influence information processing. More transitory belief-like products will be referred to as temporary appraisals.

Because the information processing framework conceives of cognitive products as phenomena that emerge from cognitive structures, temporary appraisals are thought to map onto underlying cognitive structures. So when the wife mentioned above temporarily appraises her husband as an idiot, her assessment will be linked, for example, to a belief that when it comes to clothes storage, household floors should not be favoured over cupboards, drawers, and washing baskets. As will be discussed in this thesis, the notion that a fairly simple mapping exists between cognitive products and structures has been highly influential in the CSO literature, for the statements that CSOs utter are often taken to directly reflect cognitive structures. It is the assumed simplicity of this mapping that the experimental techniques employed in this thesis will call into question.

\section{Types of Theory Regarding Child Sexual Offending}

There are many theories of child sexual offending, most of which draw upon particular paradigms for understanding human behaviour. These include behavioural (Laws \& Marshall, 1990), psychodynamic (Groth, Hobson, \& Gary, 1982), and feminist (Hermann, 1990) paradigms. To help guide theory development within this field of inquiry, Ward and Hudson (1998) have proposed that theories regarding CSO aetiology can be sorted into three theory categories:

\section{Level I (multifactorial) theories}

Multifactorial theories attempt to provide a comprehensive and integrated explanation of sexual offending. Typically they take single aetiological factors identified in the literature and weave them into explanatory models. 


\section{Level II (single-factor) theories}

Single-factor theories focus on specific factors associated with the commissioning of sexual offences. They typically explain how the factor in question differentiates sexual offenders from others and how it contributes to sexual offending behaviour. Examples include intimacy deficits (Ward, Hudson, Marshall, \& Siegert, 1995), deviant sexual arousal (Lanyon, 1991), and offence-supportive beliefs (Abel, Becker, \& CunninghamRathner, 1984).

\section{Level III (microtheory) theories}

Microtheories are essentially descriptive models of the sexual offending process. They outline how cognitive, behavioural, motivational, and contextual factors unfold across the course of the offending process (e.g., Ward, Louden, Hudson, \& Marshall, 1995). This temporal mapping of aetiological factors is often referred to as an offence chain. Microtheories, or offence chains, should provide fairly detailed accounts of what and when offence-related events occur, as well as showing how these events interact with individual characteristics to culminate in a sexual offence. For instance, the chain should illuminate the level and nature of violence or coercion involved in the offence process, the degree of planning preceding the offence and when that planning occurs, and whether or not the offence is part of an antisocial lifestyle. These factors should be interwoven with individual vulnerability factors such as social skills deficits and emotion regulation difficulties. Ultimately, microtheories ought to represent or describe what multifactor theories attempt to explain.

\section{Multifactor Theories of Child Sexual Offending}

The work documented in this thesis is primarily focussed on cognitions in CSOs and as such it explores a level II or single-factor theory. However, it must be stated that 
although CSOs' cognitions play an important role in their offending behaviour, no single factor acting in isolation can lead an individual to sexually offend. Rather, sexual offending is driven by numerous aetiological phenomena that interact in dynamic ways. Thus, before focusing squarely on CSO cognition, CSO cognition will first be considered within the context of these other phenomena. The following section considers four influential multifactor models of sexual offending and discusses the roles assigned to cognitive factors within each model. Three models relate to child sexual offending specifically (i.e., Finkelhor, 1984; Hall \& Hirschman, 1992; Ward \& Siegert, 2002) while one relates to sexual offending in general (i.e., Marshall \& Barbaree, 1990).

\section{Finkelhor's (1984) Pre-conditions Model}

According to this model, four preconditions must be met before an individual commits child sexual abuse. Finkelhor posits that these preconditions occur in a stepwise, temporal sequence.

1. The CSO must be motivated to seek sexual relations with a child. This motivation will be driven by one or more of the following:

a. he ${ }^{1}$ feels emotional congruence with the child (i.e., he identifies strongly with the child and believes the child capable of satisfying his emotional needs);

b. he experiences sexual arousal in response to the child;

c. he has limited access to other, more appropriate, sources of sexual gratification.

2. The CSO must overcome internal inhibitions against acting on his desires. While this often involves the use of alcohol and drugs, sometimes situational factors (e.g., conditions inducing high personal stress) lower inhibitions. In addition, some

\footnotetext{
${ }^{1}$ Because the vast majority of sexual aggressors are males (Pryor \& Stoller, 1994; Fergusson \& Mullen, 1999), throughout this thesis CSOs are given masculine descriptors.
} 
CSOs hold a socially-endorsed view that men are more entitled than women and children. In other words, CSOs may believe that men deserve authority and that their rights supersede those of women and children.

3. The CSO must overcome external inhibitors that make it difficult to act on his desires. In order to offend during this phase, he must create situations in which the abuse can occur. For instance, he may offer to baby-sit the targeted child.

4. Finally, the CSO must overcome or undermine potential resistance exhibited by the child. This grooming process may involve forming a friendship with the child, bribing the child with gifts and affection, or securing the child's acquiescence using violence and threats.

As this outline indicates, Finkelhor theorised that offence-facilitating cognitions can play an important role in child sexual offending, but are neither sufficient nor necessary conditions for sexual offending to occur. According to precondition two CSOs may hold the view that men are entitled to use children to meet their own needs, and this view will lower their inhibitions against sexual offending. However, these disinhibiting cognitions do not provide the motivation to offend, and instead are used by the offender to feel better about pursuing his emotionally- and sexually-derived drives. Thus, rather than being unique, CSO-specific phenomena, the disinhibiting cognitions are socially-endorsed views that play a causative role in CSOs' actions only when combined with a desire to sexually abuse.

Unfortunately, it is not clear what form these disinhibiting cognitions actually take. At times Finkelhor suggests that CSOs hold stable belief structures, acquired via social learning, that consistently lead them to interpret situations in offence-supportive ways. For instance, Finkelhor lists "male inability to identify needs of children" (p. 57) and "ideology of patriarchal prerogatives for fathers" (p. 56) as disinhibitors. Then again, he also describes these disinhibiting cognitions as if they are temporary appraisals, as 
evidenced by his referral to them as "rationalisations" (see p. 63) and self-directed “excuses" (see p. 45).

Thus, it appears that Finkelhor viewed the nature and role of CSOs' disinhibiting cognitions in two ways: first as beliefs that help to cause sexual offending by eliciting offence-supportive interpretations of events; and second as excuses utilised during a decision-making process to achieve a desired goal.

\section{Marshall and Barbaree's (1990) Integrated Theory}

According to the Integrated Theory, biological, sociological, cultural, and situational factors combine to elicit sexual offending. The theory posits that males are born with a biological predisposition to sexually aggress. During childhood, however, they learn to inhibit their aggression and regulate their emotions. Through social learning they also develop attitudes and behaviours that discourage sexual aggression. Consequently, when males experience the dramatic hormonal changes of puberty (which heighten desire for sexual activity and aggression) they are usually able to control their impulses and can meet their needs through appropriate means. However, some males fail to inhibit their sexual aggression as a result of three influences:

\section{Childhood Experiences}

Some males experience childhood adversity (e.g., poor attachment with parents, exposure to physical or sexual abuse), which leads to poor socialisation. These experiences limit the opportunity to adequately develop social skills. They also encourage self-centredness and indifference to the rights and needs of others, feelings of hostility and inadequacy, and the belief that aggression is an effective approach. Thus, there is a disruption in the social learning process by which males typically discover how to attain intimate bonds with others and how to constrain aggression. Consequently, the young man who has suffered adverse childhood experiences might use aggression to meet his emotional and sexual needs. 


\section{Sociocultural Context}

The sociocultural environment often contains misleading messages that encourage sexual aggression against women and children. Sources such as mainstream media and pornographic material can endorse beliefs that portray males as deserving dominance over women and children, or that encourage the use of violence to attain desired ends. Vulnerable males (i.e., those who have had adverse childhood experiences) are more likely to embrace these negative attitudes and beliefs because of the sense of power and superiority over others that such beliefs confer.

\section{Transitory Situational Factors}

According to the Integrated Theory, sexual offenders generally offend under circumstances that disinhibit their control. Oftentimes these circumstances will be deliberately created by the offender but this is not always the case. Males who have experienced adverse childhood experiences are more likely to be behaviourally influenced by disinhibiting circumstances. However, even well socialised males will offend if exposed to an overwhelming set of disinhibitors. Such disinhibitors include alcohol intoxication, elevated sexual arousal, heightened anger and stress, and conditions that reduce risk of crime detection, or that heighten alienation of the victim.

The Integrated Theory is broad in scope, viewing the offender as a biologicallyinstantiated being who is situated within a web of shifting environmental influences. Nevertheless, cognitions (learned from childhood experiences and interactions with the offender's social milieu) are assigned an aetiological role in the model. These offencesupportive cognitions are generally described by Marshall and Barbaree (1990) as beliefs and attitudes. Marshall and Barbaree suggest that these learned beliefs can lower inhibitions against sexual abuse by making the abuse seem less harmful, abnormal, or immoral - which presumably protects the offender's self image and minimises negative affect such as guilt. Thus it appears that sexual offenders can hold cognitions that, when 
combined with environmental and biological stressors, play a causative role in the commissioning of sexual abuse. Marshall and Barbaree do not state whether all sexual offenders necessarily hold these beliefs. However, the authors assert that “... the influence of several disinhibitors at once, particularly over an extended period, may overcome the constraints of even the most prosocial man" (p. 269). This statement implies that men can sexually offend in the absence of offence-supportive beliefs. Quite how these external factors actually disinhibit men against sexual offending is unclear, although it is possible that the authors conceived of them as altering the way that cognitive processing occurs. To summarise Marshall and Barbaree's conception of offence-relevant cognitions, they appear to propose that sexual offenders can hold beliefs that play an aetiological role in their sexual offending behaviour. However, the authors also posit that some sexual offenders commit their abuses in the absence of such beliefs.

\section{Hall and Hirschman's (1991; 1992) Quadripartite Model}

In 1992 Hall and Hirschman developed an interactive model of child sexual offending. It was built on an earlier model (Hall \& Hirschman, 1991) designed to explain sexual offending against adults. According to the Quadripartite Model (Hall \& Hirschman, 1991 ; 1992) four factors act as motivational precursors to sexual abuse:

\section{Sexual arousal}

Hall and Hirschman (1992) argue that CSOs tend to show greater than normal arousal to paedophilic stimuli. However, the authors note that not all CSOs exhibit deviant arousal. They also note that sexual arousal alone is unlikely to engender sexual offending, and will probably be accompanied by offence-supportive cognitions (see below).

\section{Offence-supportive cognitions}

Hall and Hirschman describe a number of child-related cognitions that CSOs can have which encourage sexual abuse. For example, the authors state that CSOs may think that children can benefit from abuse because it educates them about sex. In addition, they 
may think that children can initiate and enjoy sex. Hall and Hirschman (1991; 1992) call these appraisals "cognitive distortions", although they do not explicitly define what cognitive distortions are. On one hand, the authors frequently describe cognitive distortions as if they are temporary cognitive products. For example, they frequently refer to cognitive distortions as "cognitive appraisals" (Hall \& Hirschman, 1991; 1992) and also call them "rationalisations" (Hall \& Hirschman, 1992). On the other hand, the authors describe CSOs' cognitive distortions as if they are stable belief structures. For instance, in their 1991 paper Hall and Hirschman say that cognitive distortions can act as motivational precursors to sexual assaults. This implies some distortions are not so much temporary appraisals as stable cognitions that actually drive sexual offending. The authors also state that a conceptualising framework for cognitive distortions can be found in a belief based model called the Health Belief Model. In addition, when providing an example of a raperelated "cognitive distortion", the authors give a proposition that actually represents a generalised belief: "women are hostile to men and deserve to be raped" (p. 13). Again, in their 1992 paper the authors describe the following generalised belief as an example of a CSO-relevant cognitive distortion: "sexual contact with children is a method of educating them about sex" (p. 14).

\section{Emotional dyscontrol}

Hall and Hirschman state that some CSOs are motivated by anger or, more commonly, by depression to offend.

\section{Personality deficits}

According to the theory some CSOs' early experiences (e.g., childhood sexual abuse, parental neglect) may produce enduring traits that interact with the abovementioned factors to facilitate sexual offending.

In addition to being motivational precursors, the above four factors also define CSO typologies, because one factor is the dominant aetiological influence for different 
groups of CSOs. While all CSOs exhibit some level of the above four factors, less dominant factors are only mobilised via activation of the dominant factor. Hence, there are four child molester subtypes, demarcated according to which factor is dominant.

To summarise Hall and Hirschman's conceptualisation of the role/nature of cognitions in child sexual offending, all CSOs exhibit offence-supportive cognitions to some degree. These cognitions are a necessary but not sufficient factor in the commissioning of sexual offending behaviour. For some CSOs these cognitions do not play a major role and instead are contributory only when a more powerful factor activates the desire for sexual offending. For other CSOs, however, offence-supportive cognitions somehow serve as the dominant drivers of their offending. As discussed, Hall and Hirschman sometimes appear to conceptualise these so-called "cognitive distortions" as if they are belief structures, while at other times they appear to conceptualise them as temporary products.

\section{Ward and Siegert's (2002) Pathways Model}

Ward and Siegert created the Pathways model by knitting together the theories outlined earlier. According to the Pathways model, four psychological mechanisms generate the particular emotional, cognitive, sexual, and social phenomena that characterise CSOs. These are: intimacy and social skills deficits; deviant sexual scripts; emotional dysregulation; and cognitive distortions. While each mechanism contributes to every sexual offence, one mechanism is particularly influential. This primary mechanism determines the aetiological pathway a sexual offender follows. The four psychological mechanisms and their influences as primary mechanisms are outlined below.

\section{Intimacy and social skills deficits}

Ward and Siegert (2002) argue that CSOs have diminished ability to form close, intimate relationships with adults. Some may also have experienced abuse or neglect as children, and thereby have disrupted internal working models regarding relationships and 
the importance and emotional availability of others. CSOs for whom this is the primary mechanism are unable to achieve intimacy with adult partners, so they transfer their need for sex and closeness to children. These offenders believe they have a right to fulfil their sexual needs however they can. This results in a sexual arousal response to children and engenders attempts to establish adult-like relationships with children.

\section{Deviant sexual scripts}

Sexual scripts are described by Ward and Siegert (2002) as mental representations about sexual encounters that individuals develop during childhood. Essentially, scripts are types of schemas and as such are cognitive structures (Nisbett \& Ross, 1980; Schank \& Abelson, 1977). Sexual scripts guide individuals as to how, where, when, and with whom one can have sex. Ward and Siegert argue that early sexual abuse can create inappropriate scripts because it exposes children to sexual experiences before they are cognitively and emotionally able to process them. Because CSOs are more likely to have been sexually abused as children they are more likely to hold deviant scripts. CSOs who are primarily driven by this mechanism have sexual scripts wherein relationships are represented in purely sexual terms, and they hold distorted beliefs regarding relationships. Deficits in forming close adult relationships lead them to try to achieve intimacy through sexual contact with children, for these CSOs believe that children are more trustworthy, safe, and accepting than adults.

\section{Emotional dysregulation}

Emotionally dysregulated individuals have difficulty managing their emotions to achieve their goals. CSOs who are primarily driven by this mechanism sexually offend when they cannot effectively manage their negative emotions. Strong negative emotions either break down their disinhibitions concerning sex with children, or lead them to opportunistically use sex with children to elevate their mood. While they prefer sex with age-appropriate partners, they will use sex with children if access to adult partners is 
blocked. Ward and Siegert state that in these cases cognitive distortions will arise as an attempt to rationalise predatory sexual behaviours. The authors' conception of cognitive distortions is discussed below.

\section{Cognitive distortions}

Cognitive distortions are broadly defined by Ward and Siegert (2002) as "maladaptive beliefs and attitudes, and problematic thinking styles" (p. 333). Note that this definition introduces some confusion because it covers both cognitive structures (beliefs and attitudes) and processes (thinking styles). Ward and Siegert state that CSOs for whom cognitive distortions are primary drivers, "possess general pro-criminal attitudes and beliefs" (p. 338) that lead them to "disregard social norms forbidding sex with children and... exploit any opportunity for self-gratification if it presents itself"' (p. 339). This description essentially paints cognitive distortions as belief structures, rather than temporary appraisals or other cognitive processes. Ward and Siegert further reinforce the conceptualisation of cognitive distortions as cognitive structures by stating that the distorted comments CSOs make may reflect faulty schemas that hold offence-supportive content. However, Ward and Siegert also point out that cognitive distortions might simply be rationalisations CSOs put forward to excuse their actions, either to maintain self-esteem or to cast themselves in a positive light socially. At this point the authors are highlighting the fact that cognitive distortions can simply be viewed as cognitive products; things CSOs say that may or may not reflect underlying cognitive structures.

According to the Pathways model, the above four mechanisms generate four CSO categories by acting as primary drivers of sexual offending behaviour and determining the pathway along which the offending pattern unfolds. In addition, there is a fifth category that consists of CSOs who have multiple, pronounced dysfunctional mechanisms. Many of these men will have been sexually abused as children and will have consequently 
developed deviant sexual scripts. They are likely to be "pure" paedophiles who consider sexual relationships with children to be ideal.

It is important to note that although the role of the four mechanisms identified by Ward and Siegert vary according to CSO typology, all CSOs will be influenced to some degree by each mechanism. The implication is that every CSO has faulty cognitions that contribute to his offending. Thus, in the Pathways model cognitions are moved from the peripheral position assigned them, for example, in the Integrated Theory, to a position of great importance. The influential cognitions that Ward and Siegert identified in the Pathways model are: sexual scripts (which are cognitive structures); and so-called cognitive distortions. As explained, the authors often describe cognitive distortions as if they are cognitive structures (e.g., scripts and beliefs), although they also sometimes refer to them as if they are cognitive processes (e.g., thinking errors) or cognitive products (e.g., excuses). Thus, it appears that Ward and Siegert afford a key role to a range of cognitions that arise across the offence chain. It is clear that Ward and Siegert view CSOs' cognitions as playing a causative role, as they are described as giving the offender permission to offend. Because cognitive distortions are multiply defined within the Pathways model, it is unclear how one should differentiate between cognitive distortions that represent offence-supportive beliefs and those that may instead be excuses or rationalisations that arise in the aftermath of offending.

\section{What do our Multifactor Models Tell Us?}

In summary, all four major multifactor theories of sexual offending posit that CSOs' cognitions can interact with other factors to generate offending behaviour. However, the nature and extent to which these cognitions contribute to child sexual offending vary from theory to theory.

Finkelhor (1984) and Marshall and Barbaree (1990) imply that holding abnormal, offence-supportive cognitions is neither necessary nor sufficient for an individual to 
sexually offend against children. However, the more recent theories of Hall and Hirschman (1991; 1992) and Ward and Siegert (2002) suggest that all CSOs exhibit faulty, offence-supportive cognitions to some degree. Hence, theorists' perceived importance of cognitive factors in the commissioning of child sexual offences appears to have increased over time. Conceptions of what these cognitions are, however, and how they exert their influence, vary within and across theories. There is confusion within and across the models as to whether CSOs' offence-supportive cognitions are temporary products or cognitive structures. Finkelhor and Hall and Hirschman sometimes suggest that CSOs' offence-supportive cognitions are belief structures, and at other times describe them as cognitive products. Marshall and Barbaree appear predominantly to view the key offence-relevant cognitions as cognitive structures (i.e., beliefs and attitudes). Likewise, Ward and Siegert seem to view the cognitions that support sexual offending as cognitive structures (i.e., beliefs, attitudes, and scripts). However, they also describe CSOs' offencesupportive cognitions as cognitive processes (e.g., "thinking styles").

There is also variance within and across the models as to the causative role of CSOs' offence-related cognitions. At times it is unclear whether CSOs' offencesupportive cognitions help drive their desire to offend, or whether they are generated simply to justify something that is already desired. Alternatively, they may be beliefs that fail to inhibit the desire for offending, or post-hoc constructions used to diminish guilt (and perhaps maintain future offending). For instance, looking at the idea that beliefs play a causative role, Finkelhor described how beliefs endorsing male entitlement could predispose a CSO to offend, while Marshall and Barbaree claimed that learned beliefs could interact with biological and environmental factors to elicit offending. Likewise, Hall and Hirschman identified CSO beliefs regarding the perceived benefits for children of sexual activity as being motivational precursors to sexual abuse, while Ward and Siegert 
named sexual scripts and cognitive distortions as two of the four major mechanisms generating CSOs' symptoms.

On the other hand, Finkelhor painted CSOs' offence-relevant cognitions as phenomena that emerge in response to the emotional and sexual needs that really drive child sexual abuse. So while offence-supportive cognitions may be co-opted when the CSO strives to meet his needs, those cognitions are not the key aetiological factor. Similarly, Hall and Hirschman suggested that CSOs' faulty cognitions might only be mobilised when a dominant, motivating factor drives them towards offending. This question over the causative role of CSOs' offence-relevant cognitions is an important one, because its answer will further inform treatment providers as to which aspects of CSOs' internal and external worlds should receive maximum attention in treatment: if cognitions are merely co-opted in the service of overarching needs and desires they presumably do not form the main treatment target. This point will be returned to in Chapter Seven.

To summarise, our most influential multifactor models of sexual offending show considerable variation in their portrayal of cognitive factors relevant to the commissioning of child sexual abuse. It is unclear whether the abnormal cognitions of interest in the study of CSOs are structures, processes, or products. Also unclear is whether the key cognitions actually drive sexual offending, or are simply enlisted in the service of other emotional and biological drives. Finally, if cognitive structures or processes do drive child sexual offending, it is unclear whether these structures or processes play a role in the offending behaviour of all or only some CSOs. As will be shown in the following chapter, this confusion over the nature and role of CSOs' cognitions is not unique to the multi-factor theories. Rather, it is strongly mirrored in the single-factor theory literature. 


\section{Chapter Two}

\section{A Single-Factor Theory: CSOs and Offence-Supportive Beliefs}

The idea that CSOs hold faulty, offence-supportive beliefs was first posited by Abel and colleagues (Abel et al., 1984; Abel et al., 1989). In their 1984 paper, Abel et al. noted that CSOs often excuse, minimise, or justify their sexual assaults when asked to explain their behaviour. Examples provided by Abel et al. (1984) included, "A child who does not resist my sexual advances really wants to have sex with me" (p. 98) and, "Having sex with a child is a good way for an adult to teach the child about sex" (p. 99). Abel et al. referred to these statements as "cognitive distortions".

Although Abel et al. (1984) did not state the source of the term cognitive distortions they likely borrowed it from the writings of Beck $(1963 ; 1967)$. Beck (1967) described cognitive distortions as the automatic and systematic errors in thinking that characterise depressed people. Examples that Beck provided of these thinking errors included "personalisation" (a tendency to perceive oneself as causing, or being at the centre, of events) and "overgeneralisation" (perceiving a negative outcome in one situation to be predictive of negative outcomes in other situations). Beck surmised that these cognitive distortions were information processing errors that stemmed from a triad of negative beliefs that depressed people held about the self, others, and the world. Thus, according to Beck $(1963,1967)$, cognitive distortions are cognitive processes that are linked to, but not synonymous with, cognitive structures.

Unlike Beck, when Abel et al. $(1984 ; 1989)$ applied the term cognitive distortions to the sexual offending field the authors were less clear about whether distortions ought to be conceptualised as products, processes, or structures. In essence, Abel and colleagues simply used the term as a label for the seemingly inaccurate statements that CSOs make regarding their offending behaviour. In their 1984 paper, Abel et al. provided researchers with a list of the types of cognitive distortions that, based on their own clinical experience, 
the authors thought were most common in CSOs. The seven cognitive distortions categories they identified are presented in table 2.1.

Table 2.1.

Cognitive distortions categories noted in Abel, Becker, and Cunningham-Rathner (1984)

Cognitive distortions

A child who does not resist my sexual advances wants to have sex with me

Having sex with a child is a good way for adults to educate them about sex

Children do not tell others about the sexual activity because they secretly enjoy it

In the future, society will begin to accept sexual relations between adults and children

An adult who fondles a child without penetration is not harming the child

When children ask questions about sex, it is because they want sexual relations with adults

My relationship with the child is greatly enhanced through having sex with them

Although Abel et al. (1984) called the above items "cognitive distortions", they stated that the items were, “...only a sample of the various beliefs and attitudes held by adults as they involve themselves with children" (p. 101). In fact, the above list seems simply to be a series of belief statements. In other words, Abel et al. presented cognitive distortions as if they were belief structures common in CSOs, rather than thinking errors or other cognitive processes. In addition, they did not acknowledge that cognitive distortions might simply be things that CSOs tell themselves or others to create a favourable self-image or to appear more favourable socially. This view of CSOs' 
cognitive distortions as belief structures is reinforced by Abel et al.'s (1984) definition of cognitive distortions as "cognitive beliefs that support sexual involvement with children" (p. 98). Hence, Abel et al. (1984) frequently implied that they did not conceive of cognitive distortion as thinking errors, as Beck $(1963 ; 1967)$ had done, and instead thought of them as beliefs.

Unfortunately, the definition of CSOs' cognitive distortions was muddied when Abel et al., (1989) later broadened the meaning to include other phenomena such as, “... an individual's internal processes, including the justifications, perceptions and judgments used by the sex offender to rationalise his child molestation behaviour"(p. 137). In their 1989 paper Abel and colleagues not only called cognitive distortions beliefs, but also described them as cognitive processes (e.g., perceptions) and products (e.g., judgments). In fact, in their 1989 paper Abel et al. frequently described cognitive distortions as if they were things CSOs said to themselves to feel better. For instance, they stated that cognitive distortions appeared to:

“...allow the offender to justify his ongoing sexual abuse of children without the anxiety, guilt and loss of self-esteem that would usually result from an individual committing behaviours contrary to the norms of his society" (Abel et al., 1989, p.137).

Thus, with the publication of Abel et al.'s 1989 paper, the term "cognitive distortion" developed a very wide definitional reach; it could mean cognitive structures, cognitive processes, or cognitive products. Of course, in the absence of any theory or research to guide Abel and colleagues as to the factors that might underlie CSOs' cognitive distortions, it is understandable that the authors found it difficult to provide a tight definition of the construct in question.

Following publication of Abel et al.'s (1984; 1989) work, Neidigh and Krop (1992) set about listing in greater detail the types of cognitive distortions endorsed by CSOs. They asked $101 \mathrm{CSOs}$ to write answers to open-ended questions about the 
"thoughts, ideas or beliefs" (p. 210) that had contributed to their sexual offending. The authors then sorted the resultant 357 separate statements into 38 categories. The ten most commonly-endorsed statements are listed in Table 2.2.

Table 2.2.

Ten most common cognitive distortions categories and percentage of offenders producing response, as per Neidigh and Krop (1992)

\begin{tabular}{ll}
\hline Percentage & Cognitive distortions \\
\hline 25 & She enjoyed it \\
25 & This won't hurt her or affect her in any way \\
21 & This is not so bad, it's not really wrong \\
20 & I was high on drugs or alcohol at the time \\
19 & I wasn't thinking or I wouldn't have done it \\
15 & No one will ever find out so I won't get caught \\
14 & She is flirting and teasing me, she wants me to do it \\
12 & We love each other so this is ok \\
10 & She didn't say no or tell, so it must be ok with her \\
10 & There is no force involved, so this must be mutual \\
\hline
\end{tabular}

Neidigh and Krop (1992) commented that although many of the derived categories aligned with the seven cognitive distortions identified by Abel et al. (1984), some categories did not directly map onto Abel et al.'s (e.g., "No one will ever find out so I won't get caught", "I was high on alcohol or drugs at the time"). Presumably this was because Neidigh and Krop's categories were developed from actual, personal CSO statements rather than the general, clinician-derived statements Abel et al. (1984) had listed. 
By organising CSOs' statements about their offending, Neidigh and Krop (1992) provided researchers with a database of content captured by offender statements. Nevertheless, Neidigh and Krop's work did not clarify what these statements represented, or how they might be involved in the commissioning of sexual offences against children. Interestingly, while Abel et al. (1984) had referred to their list of seven cognitive distortions as beliefs or attitudes, Neidigh and Krop raised the idea that the statements recorded in their study might actually be "surface level descriptions" (p. 213) rather than representing "deeper cognitive schemas or beliefs"(p. 212). However, the authors also indicated that cognitive distortions might in fact represent beliefs because, in a confusing twist, they suggested that their 38 category statements could be compiled into a scale for assessing CSO cognition. By saying that distorted-sounding statements could be used as items for CSOs to agree or disagree with in the absence of offending cues, Neidigh and Krop implied that cognitive distortions are not mere transitory thinking errors, nor descriptions used to explain or excuse offending, but rather beliefs that CSOs hold across time.

We have so far seen that Abel et al. (1984) introduced the idea that CSOs hold beliefs that facilitate their offending and that differentiate them from others. The authors frequently referred to these beliefs as cognitive distortions. In addition, they listed some of the themes captured by these beliefs (a task soon expanded by Neidigh \& Krop, 1992). However, confusion developed when Abel et al. (1989) and Neidigh and Krop suggested that CSOs' distortions might actually reflect other processes. In short, Abel and colleagues weren't able to define the processes underlying cognitive distortions, or offence-relevant statements, although they often implied that cognitive distortions are beliefs. Because the phenomena underlying cognitive distortions remain unclear, in this thesis the meaning of cognitive distortions will be taken to simply refer to the offence-related statements that 
CSOs utter or endorse which are interpreted by others as being faulty or distorted in some way.

Other researchers have used the term cognitive distortion to refer to a raft of other cognitive structures, processes, and products. For example, cognitive distortions have been called excuses (Pollock \& Hashmall, 1991), rationalisations (Neidigh \& Krop, 1992), justifications, Abel et al., 1989), minimisations (Murphy, 1990), schemas (Malamuth \& Brown, 1994), denial (Vanhouche \& Vertommen, 1999), and defensiveness (Rogers \& Dickey, 1991). A number of authors have commented on this plethora of terms (e.g., Beech \& Mann, 2002; Geer, Estupinan, \& Manguno-Mire, 1999; Maruna \& Mann, 2006), but an agreed-upon definition has never asserted itself in the literature. This lack of consensus stems from the fact that researchers are not sure what phenomena actually drive cognitive distortions, and a lack of research into underlying factors has left psychologists unable to accurately describe the phenomena that cognitive distortions represent. Very recently it has been proposed that cognitive distortions represent judgements that CSOs make about what they believe to be true, what is desirable, and how one best explains one's actions (Ward, Keown, \& Gannon, 2007). In other words, according to this model cognitive distortions reflect belief-based, value-based, and action-based evaluations and thus are not solely driven by beliefs. Because of its recency, this model has yet to be tested and it remains to be seen whether it will be taken up by researchers and clinicians. At present, however, there is a general sense in the literature that cognitive distortions reflect offence-supportive beliefs (Gannon \& Polaschek, 2006; Marshall, Anderson, \& Fernandez, 1999b). In the following section this point will be considered in further detail. Despite the highly variable use of the cognitive distortion terms by different researchers, it must be reiterated that it has not yet been clarified which phenomena CSOs' cognitive distortions represent: all that is known is that they are marked by distorted or faultysounding statements that CSOs utter or endorse. 


\section{Cognitive Distortions as Beliefs}

Abel et al. (1984) introduced a key pair of ideas to the sexual offending literature: 1) CSOs typically hold offence-supportive beliefs; and 2) evidence for these can be found in the distorted-sounding statements CSOs endorse. This interlocking pair of ideas has increasingly become embedded in the framework from which psychologists think about and study factors driving child sexual offending. The evidence for this assertion will now be reviewed.

As was shown in Chapter One, the view that CSOs typically hold distorted beliefs - as evidenced by their cognitive distortions - is reflected in the most recent major multifactor theory of child sexual offending. Recall that in the Pathways model, Ward and Siegert (2002) frequently referred to cognitive distortions as beliefs, and they proposed that cognitive distortions play a causative role in child sexual offending. In fact, the Pathways model contends that cognitive distortions are involved in the offending pathway of every CSO. If cognitive distortions are generally thought of as being equivalent to beliefs, the universal involvement of cognitive distortions within the Pathways model suggests that the majority of CSOs hold offence-supportive beliefs of some kind.

The assumption that most or all CSOs hold static beliefs has also pervaded the writings of researchers looking at single-factor theories of child sexual offending. This is evidenced by the raft of researchers who have noted that that CSOs typically hold cognitive distortions, while directly calling those cognitive distortions "beliefs" (e.g., Blumenthal, Gudjonnson \& Burns, 1999; Bumby 1996; Howitt, 1995; Marshall et al., 1999b; Milner \& Webster, 2005; Saradjian \& Nobus, 2003; Ward et al. 997).

The concept that most CSOs hold offence-supportive beliefs and that evidence for these can be found in their seemingly distorted statements is also reflected in CSO treatment programmes. A key target of current CSO treatment programmes is to challenge 
offence-supportive beliefs (Kirsch \& Becker, 2006; Vivian-Byrne, 2004). While authors often describe this process as the modification of cognitive distortions (e.g., Becker \& Murphy, 1998; Beech \& Fisher, 2002; Marshall et al., 1999b; Marshall \& Barbaree, 1999), the same authors recommend treatment techniques that actually appear to be aimed at shifting stable beliefs. For instance, in a section entitled 'Changing Cognitive Distortions', Marshall and Barbaree (1999) stress the importance of having each programme attendee describe details of his offending so treatment providers can target his, “...misperceptions and distorted attitudes and beliefs about his victim, his offence, and about other more general issues (e.g., ... a sense of entitlement about children)" (p. 69). Marshall and Barbaree go on to explain that when "cognitive distortions" are uttered they should be challenged by group members in treatment as a way for the group to learn from each other and to, "think critically about their own beliefs, attitudes and perceptions" (p. 70). Further evidence that changing beliefs is a key target of CSO treatment programmes is evidenced by the fact that the effectiveness of such programmes is frequently assessed by measuring the extent to which attendees show response shifts on cognitive distortion questionnaires (Mann, Webster, Wakeling, \& Marshall, 2007). Such questionnaires are actually designed to measure stable offence-supportive beliefs. Whether or not they succeed at this task is an issue that will be discussed shortly.

The notion that faulty beliefs drive CSOs' cognitive distortions has become so entrenched that researchers have started theorising about the nature of those underlying beliefs. Recent writings have proposed that the beliefs which purportedly drive cognitive distortions emanate from deep cognitive structures, or schemas (e.g., Beech \& Mann, 2002; Drake, Ward, Nathan \& Lee, 2001; Mann \& Beech, 2003; Maruna \& Mann 2006; Milner \& Webster, 2005; Ward, 2000; Ward \& Keenan, 1999). This premise has been articulated most clearly in Ward's work on implicit theories (Ward, 2000; Ward \& Keenan, 1999). Drawing from cognitive/behavioural literature on the development of 
people's cognitions (e.g., Carpendale \& Chandler, 1996; Dweck, Chiu, \& Hong, 1995), Ward and Keenan proposed that the beliefs that generate cognitive distortions are not unrelated propositions, and instead are the product of underlying implicit theories (ITs). Ward (2000) described ITs as networks of interrelated beliefs and concepts that CSOs hold about the nature of their "victims, the world, and themselves" (p. 821). According to Ward, the content contained within these cognitive structures leads CSOs to interpret the world in specific, offence-supportive ways.

In order to identify the types of implicit theories that CSOs hold, Ward and Keenan (1999) analysed the types of statements that CSOs had been reported to utter in interview studies (e.g., Neidigh \& Krop, 1992), as well as the potentially distorted statements listed in questionnaire measures of offence-supportive beliefs. In other words, Ward and Keenan sought to identify the clusters of beliefs that might underlie CSOs' cognitive distortions in order to gain a picture of the cognitive content held in their implicit theories. The results led Ward and Keenan to surmise that there are five ITs encapsulating the following themes: Children as Sexual Beings, Dangerous World, Entitlement, Nature of Harm, and Uncontrollable.

The Children as Sexual Beings IT contains beliefs that children have sexual interests and desires and that they are capable of making informed decisions about sex. CSOs holding this IT are likely to misinterpret children's actions as being sexually provocative. An example cognitive distortion that might be generated by this belief cluster is, "the child was asking for sex".

Beliefs contained within the Dangerous World IT hold that the world is a hostile place full of aggressive and rejecting individuals who inflict pain and suffering on each other. There are two possible belief types that might result from a view of the world as being hostile. The first set of beliefs hold that one should withdraw into the safety afforded by relationships with children, who are viewed as innocent and accepting. An 
example of an associated distorted statement is, "children are much safer than adults". The second belief set holds that one should "fight back" against the world, manipulating and punishing those who can be dominated, and pre-emptively striking out against others before they have a chance to cause harm. An associated cognitive distortion might be, "I did it because she needed to be taught a lesson".

The Entitlement IT refers to beliefs in which the offender considers himself superior to others because of his personal characteristics or social role. For example, he may consider that as a man he is in a dominant position compared to women and children. As a consequence of this superiority his wants and needs assume higher importance than the needs of others. A distorted-sounding statement associated with this IT might be, "as a man it's my right to get my kids to do whatever I please"

Beliefs characterising the Nature of Harm IT serve to minimise or deny the harm that sexual activity can inflict on children. CSOs with this IT will sometimes attempt to justify their actions by claiming that more harmful behaviours, such as physical assault, can be inflicted against a child. A possible cognitive distortion associated with this belief cluster is, "It was only a bit of fun anyway".

Finally, the Uncontrollable IT contains beliefs that one's life is chaotic and following an uncontrollable course. The CSO who holds this IT believes that they are overwhelmed by powerful internal and external forces (e.g., sexual desire, drugs, alcohol, stress, social pressures), leaving them unable to shape their day-to-day existence. A cognitive distortion that might be generated by this cluster of beliefs is, "I wouldn't have done it if I wasn't high at the time".

Ward and Keenan (1999) did not assert that these implicit theories are universal among CSOs. For one thing, they noted that not every CSO should be expected to hold all five ITs. Rather, individuals are likely to hold different numbers of ITs. In addition, Ward and Keenan remarked that some CSOs might not hold any ITs at all. The authors stated 
that, “...although they are plausible sources of the kinds of distorted thoughts individuals who sexually offend against children exhibit, we are not suggesting that all sexual offenders hold these implicit theories" (p. 822).

Unfortunately, Ward and Keenan did not expand on how one might tell the difference between a distorted statement that represents an implicit theory and one that represents other phenomena, such as impression management strategies. To their credit Ward and Keenan acknowledged this issue, stating that,

"At this point in time we are relatively ignorant of the underlying nature of cognitive distortions... However, in the absence of any substantial research or theoretical literature on the nature of mental representations in sexual offenders... we suggest that it is a useful first step to articulate plausible implicit theories that offenders could use to explain, predict and understand the actions of victims and themselves." (p. 822)

Following on from Ward and Keenan's (1999) idea, Mann and Beech (2002) constructed a model showing the role of schema-based cognition in sexual offending. Beech and Mann defined a schema as, "a structure containing beliefs or attitudes that follow a similar theme or pattern and that have developed as a result of trying to make sense of early life experiences" (p. 145). According to Mann and Beech's model, sexual offenders' dysfunctional schemas lead them to process ambiguous or negative life events in such a way that hostile interpretations are produced. When these cognitive products combine with other sexual offending-related factors (e.g., impulsivity, deviant sexual interests, lack of intimacy) the sexual offender makes a choice to engage in sexually abusive behaviour. Thus, Beech and Mann (2002) developed a model in which dysfunctional schemas are not the primary drivers of sexual offending, but are instead 
components that, when combined with a range of relevant risk factors, will heighten the likelihood of sexual assault occurring.

Whether CSOs' offence-supportive beliefs are best viewed as being isolated structures or as being linked in with larger, more pervasive schematic structures is a matter currently under consideration within the sexual offending field (Gannon, Keown, \& Rose, in press). What is evident, however, is that the hypothesis that CSOs hold offencesupportive beliefs in some form or another has gained widespread acceptance. Naturally, this acceptance raises a very important question... what evidence is there to support the hypothesis? In the following chapter this question will be explored in depth. 


\section{Chapter Three}

\section{What's in a Measure? Research Underpinning the Notion of Offence- Supportive Beliefs}

As mentioned earlier, the hypothesis that CSOs hold offence-supportive beliefs certainly has intuitive appeal. However, no matter how seemingly appealing a premise is, in the field of science we must critically consider the evidence that runs for and against it. Typically, researchers seeking information about CSOs' offence-supportive beliefs have conducted two types of inquiry. First, researchers have interviewed CSOs about their offences and identified response statements that sound distorted or inaccurate. These socalled cognitive distortions have usually been taken to reflect offence-supportive beliefs. Second, researchers have created questionnaires for CSOs in which offence-related propositions are listed, such as, "A child won't have sex with an adult unless the child wants to" (Abel and Becker Cognitions Scale; Abel et al., 1989). CSOs are asked to rate the extent to which they agree with each item. If CSOs indicate greater mean agreement with questionnaire items than comparison groups this difference is assumed to demonstrate that CSOs hold enduring, context-independent cognitions that distinguish them from non-abusive individuals. In other words, those CSOs are usually thought to believe questionnaire items to be true.

The subsequent section will review interview and questionnaire studies to consider the extent to which CSOs utter or endorse cognitive distortions. Information gathered from these two sources will be discussed, and the significance of that evidence in terms of CSOs' beliefs will be considered. Attention will then turn to the cognitive experiment, an underutilised but potentially valuable method for assessing sexual offender cognition.

\section{Interview Studies of CSO Statements}

As discussed, Abel et al. (1984) were the first to organize the content of CSOs' post-offence statements. The fact that these statements were interpreted as evidence of 
faulty beliefs is apparent in the authors' assertion that the statements represented, "beliefs and attitudes held by adults as they involve themselves with children" (p. 101). Following Abel et al.'s work, Neidigh and Krop (1992) adopted a more formal approach to recording and categorizing CSOs' post-offence statements. Using a written questionnaire format, they collected 357 statements which were assigned to 38 differently themed categories by three judges who showed high levels of interrater agreement. Neidigh and Krop were less certain about whether the collected statements represented beliefs. While there was some suggestion from Neidigh and Krop that they might reflect beliefs, the authors also posited that the recorded statements might represent "surface level cognitions" (p. 211) and "conscious self talk" (p. 211) rather than "deeper attitudes and beliefs" (p. 211). The authors made this comment after examining their participants' scores on the ABCS. Neidigh and Krop found that their participants endorsed an average of 1.8 distortions on the ABCS. This was a substantially lower number than the average 3.5 distortions that participants endorsed when being interviewed. Because CSOs appeared more distorted when being interviewed, and because CSO questionnaires are essentially designed to assess entrenched beliefs, Neidigh and Krop proposed that their participants' interview statements may have reflected temporary, situation-specific appraisals rather than beliefs. Subsequent authors (Gannon \& Polaschek, 2006) have pointed out that CSOs might also reveal more distortions when being interviewed because their belief structures are primed by in-depth discussions about their offences, increasing the online availability of their offence-relevant cognitions.

Hartley (1998) interviewed eight intrafamilial CSOs (i.e., CSOs who, unlike extrafamilial CSOs, have only offended against family members). Hartley stated that the aim of this study was to investigate how intrafamilial offenders use cognitive distortions to overcome their inhibitions against sexual offending. Cognitive distortions were described by Hartley as CSOs' faulty "thoughts, perceptions, beliefs, and attitudes" (p. 25). The 
study was based on Finkelhor's (1984) premise that CSOs must surmount internal inhibitions in order to commit sexual abuse. The so-called cognitive distortions that CSOs in this study provided were grouped by Hartley into four categories. These were: cognitions related to sociocultural factors (i.e., beliefs that society tolerates child sexual abuse in certain circumstances); cognitions used to reduce fear of disclosure (i.e., selfreassuring thoughts that the abuse will go undetected); Cognitions used to diminish responsibility (i.e., beliefs that the abuse is unplanned or not harmful); and cognitions related to permission seeking (i.e., beliefs the child could consent to the abuse). Hartley concluded that the statements uttered by CSOs in her study demonstrated that cognitive distortions are not only post-hoc rationalizations, but also represent pre-offence cognitions that lower inhibitions against offending.

In another interview study of CSOs' cognitive distortions, Ward et al. (1998) conducted a grounded theory analysis of assessment interviews held with 20 intakes at a CSO treatment programme. Within each interview, interviewees explained what motivated them and how they overcame internal inhibitions, external inhibitions, and victim resistance. Their statements were broken down into a series of so-called meaning units and Ward et al. hypothesised that some of these statements revealed information about the belief content held within CSOs' cognitive structures. However, meaning units were also found to reveal information about the offence chain. That is, they revealed information about CSOs' cognitive operations (e.g., descriptions, denials, or minimisations of the offence) and their styles of disclosing information (i.e., detailed, euphemistic, concrete, and passive). Thus, although Ward et al. used the content of CSOs' utterances to categorise pre-existing offence-supportive beliefs, they also warned that CSOs' reports may be affected by other processes. Setting aside issues about whether Ward et al.'s model is a fruitful way of categorising offender cognition, it is significant that the authors 
stressed the importance for researchers and clinicians to be aware of CSOs' cognitive operations and meta-level variables when analysing their interview statements.

More recently, Saradjian and Nobus (2003) questioned 14 CSO clergymen about their offences. The authors did this with the aim of identifying content held within the clergymen's beliefs, as well as the processes by which those pre-existing beliefs could "shape and affect subsequent judgments and behaviour" (p. 908). They detected ten categories of cognitive content, many of which, as Gannon and Polaschek (2006) have pointed out, were similar to Ward and Keenan's (1999) implicit theories (e.g., "Teenage boys are full of sex, they want it": Children as Sexual Beings). Saradjian and Nobus concluded that clergy CSOs used these beliefs both prior to the offences to overcome inhibition, and after the offences to reduce self guilt and maintain a positive self image. The authors reported that CSOs in their study held similar beliefs to other CSOs, although religious beliefs were also part of their offence-supportive thinking (e.g., God allowed it to happen).

Finally, Marziano, Ward, Beech, and Pattison (2006) interviewed 22 CSOs to group any resultant offence-supportive content into one of the five ITs described in Chapter Two. CSOs were questioned at length about their offences and the interview transcripts were coded for semantic meaning units. Of the 6,497 resultant meaning units, $41 \%$ were classified as cognitive distortions and allocated to an IT category. CSOs were found to most frequently endorse Children as Sexual Beings (28\%), followed by Uncontrollable (26\%), Dangerous World (22\%), Nature of Harm (14\%), and Entitlement (10\%). Despite the fact that Ward and Keenan (1999) contended that not all CSOs would hold ITs and that some CSOs would hold only one or two ITs, Marziano et al. reported that all 22 CSOs endorsed cognitive distortions that fitted either four or five IT categories. The authors took this remarkably high level of IT-themed cognitive distortion endorsement (along with the fact that no other so-called "core beliefs" were identified) to 
strongly suggest that CSOs do in fact hold ITs. Marziano et al. did not raise the possibility that some of the statements made by participants may have reflected phenomena other than underlying beliefs.

In summary, the interview studies reviewed above have all found that CSOs utter cognitive distortions when they are interviewed about their offences. In the only study where the rate of such utterances was compared with endorsement of cognitive distortion questionnaire items (i.e., Neidigh \& Krop, 1992) a much higher rate of endorsement was found using the interview technique. Taken together, these results suggest that interview studies reliably find evidence to support the hypothesis that CSOs generally exhibit cognitive distortions. Less clear, however, is what these distortions mean. While they are generally presented as evidence of faulty beliefs, the authors of two interview studies (Neidigh \& Krop, 1992; Ward et al., 1998) did warn that CSOs' cognitive products may not necessarily represent belief structures.

In recent years these warnings have been echoed by a number of researchers who have raised questions about the nature of cognitive distortions and underlying phenomena (e.g., Beech \& Mann, 2002; Gannon \& Polaschek, 2006; Maruna \& Mann, 2006, Neidigh \& Krop, 1992; Segal \& Stermac, 1990). Recall that the CSOs who Neidigh and Krop studied endorsed two times as many cognitive distortion items when being interviewed than when answering a questionnaire. This difference could perhaps be explained by the notion that CSOs who are asked to account for their actions sometimes produce justifications, minimizations, and denial, to deflect self-criticism or the opprobrium of others. Alternatively, when asked to explain their offending CSOs may report temporary, situation-bound appraisals that they engaged in during the time of their offending. These appraisals could have been specific, transitory cognitions that were generated through the interaction of environmental factors and individual emotional and neuropsychological drivers (see Ward \& Beech, 2006). In fact, the idea that situational variables might 
overcome more entrenched cognitions has been applied by several authors to the sexual offending field. For example, Howells, Day, and Wright (2004), Gannon and Polaschek (2006), and Ward, Hudson and Marshall (1995) have all noted that the concept of cognitive deconstruction can be called upon when accounting for CSOs' sexually abusive actions. Cognitive deconstruction is a construct that was originally identified by Baumeister (1989, 1990). It is essentially a shift from abstract, higher levels of processing to more concrete, stimuli-driven ones. This attentional shift occurs when people are in the midst of stressful circumstances. It is thought to be a self-protective strategy that temporarily screens people from self awareness, guilt and shame. When a person enters a cognitively deconstructed state their self-concept becomes less integrated and meaningful, they focus on immediate rather than long-term goals, their thinking becomes less abstract and more concrete, their self-standards are less influential, and their inhibitions and their capacity to feel guilt diminishes. Baumeister (1990) contended that individuals can oscillate between this state and a more aware and integrated one.

If this idea is applied to CSOs (as was first done by Ward et al., 1995), one can see that an individual feeling high levels of negative affect, for instance, might feel a strong drive to achieve intimacy through sexual contact with a child. Although the CSO may believe that adult-child sexual contact is unacceptable, when he enters a cognitively deconstructed state his beliefs have little influence on his behaviour. In the cognitively deconstructed state his attention is directed to isolated cues, and because his processing has become less abstract he fails to appraise those cues within the context of their broader meaning. So, for example, if his victim moves about in such a way that her underwear shows he could interpret this as a sign of sexual enticement, failing to acknowledge that his victim's action actually stems from an innocent lack of self-awareness. Deaf to his own beliefs, he is now capable of performing acts that are unconsciously aimed at alleviating his emotional pain. Once self awareness returns the individual might then seek 
some way of neutralising the attendant guilt. Thus, in his mind he generates cognitive distortions, which in this case would be self-soothing statements designed to minimize the perceived harm done, or his sense of personal responsibility.

At present, the notion of cognitive deconstruction seems to sit rather uncomfortably with recent literature on child sexual offending, and it is a topic that is not often raised by researchers. This may be because it is hard to align with the view that most or all CSOs have abnormal belief structures. After all, if one holds that CSOs have exceptional beliefs that enlist pro-offending motivations, what need is there for a theory that says sexual offending can occur in the context of exceptional, belief-subsuming circumstances? The concept does, however, align more with the older theories of Finkelhor (1984) and Marshall and Barbaree (1990). Recall that Finkelhor claimed CSOs offend only in situations when their internal inhibitions have been lowered, implying that offenders can actually offend in spite of, not because of, their beliefs. In a similar vein, Marshall and Barbaree said sex offenders generally offend under circumstances that disinhibit their control. The authors contended that even well socialised males can offend if exposed to specific circumstances and very strong stressors, a notion that clearly sits well with the idea that CSOs might offend when in a cognitively deconstructed state.

The cognitive deconstruction concept raises the possibility that cognitive distortions are sometimes things CSOs say to themselves to neutralize post-offence guilt. Alternatively, cognitive distortions could represent attempts on the part of CSOs to construct a coherent-sounding account of their motivations and decisions. Interviewers often ask CSOs to provide explanations relevant to their offending and to report on their internal states during the offending period. Usually this requires the speaker to recall events that happened some considerable time ago. Providing his memory is up to this challenge, the speaker must then report on internal states that he may very well have not been aware of at the time. As explained earlier, humans often use schemas to guide where 
we direct our attention, how we perceive and interpret stimuli, and how we recall information. Yet information regarding the contents of those schemas is not something that humans have ready access to (Beck, 1996; Nisbett \& Wilson, 1977). In fact, humans are notoriously bad at understanding their own motivations (Kunda, 1999). As an example, participants in studies have been shown to often make moral judgements based on intuitive, emotionally-driven appraisals. Yet when asked to explain how they made their judgements those people generally claim to have made rational decisions, citing culturally-sanctioned reasons for choosing a particular response (Haidt, 2001). People commonly construct these types of rationalisations despite the fact that the vast majority of human psychological processes occur outside of conscious awareness (Bargh \& Chartrand, 1999; Greenwald \& Banaji, 1995). In particular, humans have little or no introspective access to higher order cognitive processes such as those used in problem solving, evaluation, judgment, and action selection (Nisbett \& Wilson, 1977). As Nisbett and Wilson explain, humans are often unaware of their own responses as they make them. Likewise, humans are generally unaware that stimuli have heavily influenced their responses, or even that the influential stimuli are present in their environment. Although individuals are capable of adopting an effortful, data-driven processing style (Fiske \& Taylor, 1991), even when making a conscious effort to monitor cognitions humans can still be driven by factors that lie outside awareness. For instance, Bargh and Chartrand reported that when individuals try to make conscious, effortful judgments about others, unbeknownst to these individuals their judgments have already been formed on the basis of rapid, unintentional evaluations..

Despite the many difficulties involved in trying to report on one's motivations and decision-making processes, the CSO who is asked to explain his offending may produce a plausible-sounding account. While this could indicate that he is providing an accurate description of the offence process, it is more likely that he is playing storyteller, weaving 
together fact and fiction in order to create a coherent account of his offending. Malle (2006) notes that when people engage in undesirable or unusual behaviours it is very difficult for them to admit ignorance of their own reasons. Likewise, listeners often want to hear excuses that explain behaviour in a compelling way (Malle, 2006). Thus, the CSO being interviewed may attempt to construct a perfectly rational, yet nevertheless faulty, causal history that either reduces his discomfort at not knowing why he committed his crimes, or that gives the interviewer an explanation they seem to want.

In addition to the above possibilities, some cognitive distortions may be statements that CSOs utter in order to project a more positive image of themselves to others (see Gannon \& Polaschek, 2006; Maruna \& Mann, 2006). According to Maruna and Mann, it is common for people, especially westerners, to provide excuses when asked to explain their misdeeds. That is, humans have a tendency to cite external causes and mitigating circumstances when accounting for their bad behaviour. In particular, humans tend to deny planning or intentionality when explaining misdeeds, both to minimise their own guilt (Baumeister \& Wotman, 1992) and to enhance their reputation (Maruna \& Mann, 2006). Such excuses can actually play an adaptive role, because they allow the speaker to maintain healthy self-esteem levels and can strengthen social ties by indicating a desire to follow social norms (Maruna \& Mann, 2006). In short, excuse-making is both common and adaptive... unless, it seems, one has sexually offended against children. In this case, individuals who engage in excuse-making are usually pathologised.

Finally, some cognitive distortions might simply be fairly accurate event descriptions. When an offender says something like, "I was too drunk at the time to think straight" or, "I was depressed" or, "she hopped into the bed and started cuddling up to me" his descriptions might merely be that: descriptions. The offender is not necessarily excusing his behaviour. Rather, he may be trying to give his listener information about external and internal forces acting on him during the offending period. Maruna and Mann 
(2006) and Mann and Hollin (2007) have noted that far from being misinterpretations that ought to be challenged, such statements could very well signal dynamic risk factors the offender needs to address in treatment. Regardless, there has been a tendency in clinical practice to declare almost any causal explanation that offenders extend regarding their behaviour as being distorted (Maruna \& Mann, 2006).

In summary, the seemingly distorted-sounding statements that CSOs utter in interviews could arise from a raft of phenomena. They might, as is often suggested, be direct descriptions of abnormal content held within belief structures or schemas. On the other hand, they might represent CSOs' attempts to make logical sense of behaviour that was not enacted for logical reasons, or attempts to introspect on motivations that lie beyond the reach of introspection. Alternatively, they might be direct reports about temporary appraisals the offender made when offending, or self-soothing statements that kick into gear when the offender contemplates the guilt-inducing fact that he abused a child. They might be excuses the offender extends to his listener because he wants to assure the listener he is a likeable person after all. Then again, cognitive distortions might sometimes be matter-of-fact descriptions that are not designed to excuse or justify anything. Unfortunately, the research literature contains scant discussion about these competing phenomena, how psychologists might tell the difference between them, or what the treatment implications are that they yield.

\section{Questionnaire Studies of CSO Statements}

Despite warnings that CSOs' cognitive products might not represent beliefs, researchers have nevertheless used the semantic content derived from CSOs' interview studies to develop questionnaire measures of offence-supportive beliefs. Typically, these questionnaire measures are administered to CSOs and offender or community control groups under confidential or anonymous conditions. The underlying rationale is that if 
CSOs hold stable, non-situational, offence-supportive beliefs their answers should be statistically distinct from those provided by non-CSOs (Ward et al., 1997).

The first such questionnaire was developed by Abel, Becker, CunninghamRathner, Rouleau, Kaplan, and Reich (1984). Called the Abel and Becker Cognitions Scale $(A B C S)$, this 29-item scale was simply based on the authors' clinical experience in verbally assessing CSOs. Abel et al. (1989) found the scale to have acceptable psychometric properties (test-retest reliability of .76 over three weeks and internal consistency ranging from .59 to .82 across six extracted factors). Examples of items include "A child who doesn't physically resist an adult's sexual advances, really wants to have sex with the adult" and, "Having sex with the child is a good way for an adult to teach the child about sex." Items are scored on a 5-point Likert scale from 1 (Strongly agree) to 5 (Strongly disagree). The scale was found by Abel et al. (1989) to have six factors, which were labelled (see pp. 144-145) as follows: "Child-adult sex helps the child"; "Children initiate child-adult sex for specific reasons"; "Adults initiate child-adult sex for specific reasons"; "The child's behaviour shows their desire for child-adult sex"; "Adults can predict when child-adult sex will damage the child in the future"; "Child-adult sex is or will be acceptable in society". Abel et al. (1989) noted that ABCS items were transparent, meaning that no attempt was made to disguise the questionnaire's purpose.

Despite this transparency the instrument has been used with a reasonable degree of success in various studies. Abel et al. (1989) used the ABCS to compare responses from 240 CSOs, 48 non-CSO paraphilics (i.e., men who exhibited other forms of sexual deviancy), and 86 community controls. CSOs were found to endorse significantly more items than community controls on all six factors, although no factor statistically distinguished CSOs from paraphilics. Stermac and Segal (1989) also used the ABCS to compare 20 CSOs with 17 rapists, 35 clinicians and 94 community controls. CSOs demonstrated significantly higher endorsement of items than any other group. In 1995 
Hayashino, Wurtle, and Klebe examined whether the ABCS could distinguish extrafamilial CSOs from intrafamilial CSOs, community controls, offender controls and rapists. In line with the authors' expectations, extrafamilial CSOs demonstrated significantly higher levels of agreement than all other groups. Interestingly, intrafamilial CSOs did not respond differently than rapists or other control groups. Researchers have suggested that extrafamilial CSOs hold higher levels of distorted beliefs than intrafamilial CSOs (Fisher, Beech, \& Browne, 1999; Vanhouche \& Vertommen, 1999; Ward, 2000), which may account for Hayashino, Wurtle, and Klebe's findings. Extrafamilial CSOs are hypothesised to be more distorted than intrafamilials because they tend to have more victims over a longer offending period and therefore have more opportunities to practice interpreting their world in a distorted manner and thus to strengthen their offencesupportive beliefs or schemas.

In contrast to Abel et al.'s (1989) and Stermac \& Segal's (1989) findings, Beech et al. (1999) were unable to distinguish between CSOs and nonoffenders using the ABCS. The authors dealt with this unexpected result by removing the two-thirds of the CSO sample that they classed as "low-deviancy" (i.e., showing some combination of having fewer victims, picking victims of only one gender, and offending within their own families), and including only "high-deviancy" CSOs in the analysis. At this point a significant difference was found between CSOs and nonoffenders. Finally, Tierney and McCabe (2001) found that the ABCS statistically discriminated between CSOs and community controls, but did not distinguish CSOs from adult sexual offenders or offender controls.

Taken together, these studies indicate that the ABCS can often distinguish CSOs' responses from those provided by community controls, but that it is less successful at differentiating CSOs from other offender groups. While Abel et al. (1989) did not measure socially desirable responding when examining the ABCS, questionnaire measurement of 
social desirability was included in other ABCS studies (i.e., Hayashino, Wurtle, \& Klebe, 1995; Stermac \& Segal, 1989 ; Tierney \& McCabe, 2001), all of which eliminated it as an influence on ABCS results. In the case of Beech et al.'s (1999) study neither high deviancy nor low deviancy CSOs were found to differ from nonoffenders in terms of socially desirable responding. Nevertheless, it has been suggested that discriminative ability of the ABCS may have been limited by transparency of items (e.g., Blumenthal et al., 1999; Bumby, 1996; Tierney \& McCabe, 2001). In other words, researchers have surmised that rather than providing completely honest responses, some CSO participants engage in "socially desirable responding", or "faking good", thereby muddying group differences between CSOs and controls.

To minimize the potentially confounding effects of socially desirable responding, Bumby (1996) designed the MOLEST, a questionnaire measure that prevents CSOs from being neutral about their beliefs. Responses to MOLEST items are made on a 4-point Likert scale, removing the opportunity for respondents to choose a "neutral" response. Bumby reported that this 38 -item scale has excellent psychometric properties, with an internal consistency of .97 and test-retest reliability of .84 over two weeks. In addition, the scale has a low correlation with the Marlow-Crowne Social Desirability Scale (MSDS; Crowne \& Marlowe, 1960). Example items are, "Sometimes, touching a child sexually is a way to show love and affection" and, "Some children can act very seductively".

Bumby (1996) compared the MOLEST responses of 44 intrafamilial CSOs, 25 rapists, and 20 non-sexual offender controls. All participants were incarcerated in a maximum security correctional facility. In a successful demonstration of the instrument's discriminative power, CSOs were found to endorse significantly more distorted statements than rapists and non-sexual offender controls, while the offender control groups could not be statistically differentiated from each other. Other researchers have since found the MOLEST to statistically discriminate extrafamilial CSOs from offender and community 
comparisons (Marshall, Marshall, Sachdev, \& Kruger, 2003), extrafamilial CSOs from rapists (Arkowitz \& Vess, 2003), CSOs from sexual offenders against adults (Blumenthal et al., 1999) and CSOs from rapists and violent offenders (Feelgood, Cortini \& Thompson, 2005). Most recently, Pervan and Hunter (2007) used the MOLEST to compare the cognitions of 64 CSOs, 36 rapists, 25 violent offenders, and 14 male university students. Contrary to the ABCS findings reported by Hayashino et al. (1994) and Fisher et al. (1999), Pervan and Hunter found no significant differences between extrafamilial and intrafamilial CSOs' scores, and for this reason they did not analyse the CSO subtypes separately. It was found that CSOs attained significantly higher scores than rapists and violent offenders, but did not differ from university students. Despite being unable to distinguish between CSOs and university males, and despite the fact that their method simply involved using propositional belief statements to measure cognitive distortions, Pervan and Hunter concluded that, "the clear group differences... reaffirms the notion that sexual offenders are prone to misinterpret or reinterpret social perceptions in a way that justifies, defends, and maintains their offending behaviour" (p. 88).

At around the time that Bumby was developing his questionnaire, Hanson, Gizzarelli, and Scott (1994) created the Hanson Sex Attitude Questionnaire (HSAQ). This 47-item instrument was constructed to assess the offence-supportive beliefs of intrafamilial CSOs. The HSAQ contains six subscales (Sexual Entitlement, Children are Sexual, Sexual Harm, Frustration, Affairs, Sex/Affection Confusion), and items are answered using a 5-point Likert scale. Hanson et al. reported that all subscales demonstrated acceptable alphas ranging from .62 to .92 . Test-retest reliability was not described. Examples items are, "Everyone is entitled to sex" (Sexual Entitlement), "Some children like to sexually tease me" (Sexy Children), "Children can easily forgive parents if they have sex with them" (Sexual Harm), "I am often sexually frustrated" (Frustration), "A man can have sex outside marriage and still love his wife" (Affairs), and "Sex makes 
all relationships stronger" (Sex/Affection Confusion). Hanson et al. found that Sexual Entitlement, Children are Sexual, and Sexual Harm subscales statistically distinguished intrafamilial CSOs from male batterers and community controls. However, Frustration, Affairs, and Sex/Affection Confusion did not discriminate CSOs from controls, even though analysis of covariance suggested that these scales were not subject to socially desirable responding.

More recently, a 22-item cognitive distortion questionnaire called the Child Molester Scale was devised (CMS; McGrath, Cann, \& Konopasky, 1998). Its items are comprised of statements that justify sexualized adult-child interactions such as, "Play wrestling with children which includes some tickling and touching between the child's legs is not sexual assault" and, "Children usually outgrow any problems resulting from a sexual experience they had as a child". McGrath et al. posited that wording distorted statements in a justificatory way would reduce socially desirable responding. McGrath et al. also included distracter items to obscure the purpose of the instrument. Item responses are indicated using a 5-point Likert scale.

To date, the CMS has demonstrated only limited ability to distinguish CSOs from controls. In the first study of its discriminative abilities McGrath et al. (1998) collected scale responses from 30 CSOs, 30 offender controls, and 30 male university students under conditions of complete anonymity. These response were compared with those of a group of 44 CSOs who were about to be assessed for parole and who were not assured anonymity. As expected, CSOs overall and CSOs in the parole condition endorsed significantly more CMS items than the university males, and CSOs who were assured anonymity showed higher levels of item endorsement than CSOs in the parole condition. However, CSOs in the parole condition could not be differentiated from offender controls, even though the parole-eligible CSOs had good reason to respond to CMS items in a socially desirable way. In another study where confidentially of responses to the CMS was 
assured (see Gannon \& Polashchek, 2006, for a review), Tierney and McCabe (2001) found that CSOs' responses to CMS items did not significantly differ from those of nonsexual offender controls and sexual offenders against adults.

Overall, questionnaires that ask respondents to indicate their belief levels across lists of cognitive distortions tend to discriminate between CSOs and comparison groups. Therefore, on the face of it, questionnaire studies would seem to suggest that CSOs often hold stable, offence-supportive beliefs that set them apart from other offenders and nonoffenders. However, looking slightly below the surface, there is an intriguing pattern lurking in the findings of these studies: even when CSOs demonstrate greater endorsement of distorted-sounding statements on questionnaires, they do not actually agree with those statements. Closer examination of scores shows that CSOs' Likert scale ratings typically tend towards the disagree option. For example, Langevin (1991) found that almost $93 \%$ of CSOs' responses to the ABCS fell into the disagree or strongly disagree range. In other investigations of the ABCS, Hayashino et al. (1994) and Tierney and McCabe (2001) reported that CSO respondents disagreed with statements overall. Similarly, studies using the MOLEST have indicated that CSOs do not endorse cognitive distortion items. For instance, CSOs' responses in Arkowitz and Vess' (2003) study were skewed towards the disagree option and Marshall et al. (2003) found a mean CSO total response score of 66 within a possible range of 38-152, again indicating that CSOs disagreed with items. Likewise, Blumenthal et al. (1999) found that CSOs scored a mean total of 80.1 on the MOLEST. With MOLEST scores of 76 indicating disagreement, and scores of 114 indicating agreement, Blumenthal et al.'s CSOs clearly tended towards disagreement with MOLEST items. In addition, Feelgood et al. (2005) reported that CSOs' responses to the MOLEST were in the slightly disagree range. Finally, Pervan and Hunter (2007) found that CSOs attained a mean total score of 64, indicating clear disagreement with items. Nevertheless, the authors went on to conclude that CSOs' response indicated "the 
endorsement of cognitive distortions" (p. 88). It seems strange, to say the least, that one might interpret disagreement with items as a sign that those items are being endorsed. Most psychologists would challenge any sexual offender who similarly mistook disagreement for affirmation!

To summarise, then, even when questionnaire measures of offence-supportive beliefs find differences between CSOs and others, the clinical significance of those differences is debatable in light of the fact that CSOs tend to disagree with questionnaire items. One has to ask whether slight differences in disagreement levels between CSOs and others offers support for the hypothesis that CSOs hold distorted, abnormal beliefs.

When confronted with this paradox, or with nonsignificant comparisons between CSOs' and controls' questionnaire responses, some researchers have argued that the results indicate socially desirable responding on the part of the CSOs. For instance, when Langevin (1991) found that only $7.2 \%$ of CSOs' ABCS items fell into the neutral to strongly agree range, he argued that these findings were best accounted for by the likelihood that CSOs were engaging in socially desirable responding. This argument was extended despite the fact that no measure of social desirability was included in Langevin's study. Likewise, Fisher et al. (1999) suggested that their own study may not have distinguished between controls and intrafamilial/low deviancy CSOs because those CSOs faked good when answering the ABCS. Fisher et al. extended the same argument to account for the fact that Hayashino et al.'s (1995) study did not differentiate intrafamilial CSOs from controls. Finally, Kolton, Boer, and Boer (2001) interpreted null findings using a modified version of the ABSC as evidence that only when CSOs respond using the strongly disagree option should their responses be viewed as distortion-free. Amazingly, the authors argued that all other CSO responses ranging from disagree to strongly agree should count as evidence of distorted beliefs! 
Of course, these approaches represent a tautology: when CSOs' responses differ from controls' the results are accepted as support for the hypothesis that CSOs hold distorted beliefs, but when differences are not detected the results are dismissed as indicating that CSO respondents faked good. By adopting such a stance it becomes difficult, if not impossible, to produce questionnaire results that negate the offencesupportive belief hypothesis. Importantly, research has produced little or no evidence that CSOs do feign disagreement when answering questionnaire items. This lack of evidence rests on the fact that social desirability bias is either not measured when questionnaire assessments of offence-supportive beliefs are administered, or that when it is measured it is generally not found to predict participants' responses (Gannon \& Polaschek, 2006). For instance, Arkowitz and Vess (2003) found that although CSOs' MOLEST responses were skewed towards disagreement, those responses did not significantly correlate with general social desirability. Blumenthal et al. (1999) also reported that although CSOs' mean total scores indicated disagreement, social desirability did not appear to affect their MOLEST responses. Three recent studies have tried to shed new light on this issue by investigating CSOs' tendency to fake good using experimental techniques. Two studies (Gannon, 2006; Gannon \& Polaschek, 2005) found evidence CSOs weren't faking good, while one study (Gannon, Keown \& Polaschek, 2007) found evidence they were. On balance then, the body of evidence seems to suggest that socially desirable responding does not greatly interfere with CSOs' questionnaire responses. Nevertheless, when CSOs confound researchers' hypotheses by disagreeing with questionnaire items or answering questionnaires similarly to controls, there is a tendency to surmise that those CSOs have hidden their distorted beliefs away from the eyes of prying researchers. Strangely, researchers do not seem to consider the alternative possibility that control groups, aware of the widespread abhorrence of child sexual abuse, might sometimes fake good on CSO questionnaires. It is, after all, not out of the question that controls could sometimes answer 
such questionnaires defensively, exaggerating their disagreement for fear of being suspected capable of child sexual offending. Were this scenario the case, it would account for the mixed pattern of questionnaire findings equally well.

There is another reason to query the idea that questionnaires provide evidence that CSOs hold offence-supportive beliefs. As was the case with the interview method, the questionnaire method assumes that CSOs' faulty beliefs are chronically accessible and readily open to offenders' introspection. Yet research has suggested that this assumption may well be unwarranted: humans are often unable to accurately introspect on the contents of their cognitive structures. If it is difficult for people to accurately report their beliefs they may instead try to logically deduce what those beliefs are. When rating an item like, "Children who have been involved in sexual activity with an adult will eventually get over it and go on with their lives" an offender who has abused a child may reason that he cannot hold a competing belief, or he wouldn't have committed the crime. As noted earlier, it seems intuitively wrong that someone with a belief that sexual abuse is harmful might nevertheless molest a child. It has been shown, however, that the CSO who makes this conjecture could be wrong; people can and do behave in ways that violate their own belief systems.

We have so far seen that interviews and questionnaires have been used to study the hypothesis that CSOs hold offence-supportive beliefs, and they have revealed data that has been interpreted as support for that hypothesis. Yet both interviews and questionnaires record cognitive products without shedding light on the underlying phenomena that drive CSOs' responses. This is not to say that interview and questionnaire approaches are not valuable tools when studying CSOs; they certainly have allowed theoreticians and clinicians to hypothesise about the nature and content of CSOs' offence-relevant cognitions. However, there is a need for these techniques to be complimented by more sophisticated, implicit methods that use phenomena other than cognitive products to 
investigate CSOs' beliefs. In the section below studies that have attempted to measure cognitive processes, rather than products, will be reviewed.

\section{A New Paradigm: Information Processing Studies}

As discussed, people are not always able to consciously access information about their own cognitive processes and contents. Even if they successfully introspect on these cognitive phenomena, there is no guarantee that they can accurately articulate their thoughts, or that they will choose to do so honestly. To overcome these issues, cognitive scientists have developed experiments that minimise both the opportunity for participants to censure their responses and the need for them to introspect on implicit cognitions. These experiments are designed to allow covert observation of the ways in which humans automatically process information. For example, the time that it takes for participants to automatically respond to stimuli is surreptitiously recorded, or their memory for presented information is tested. The observations provide important clues about participants' underlying cognitive structures and contents. Underpinning the design of such experiments is the principle that cognitive structures and their contents strongly influence the way that humans attend to, encode, store, and react to environmental stimuli. As Greenwald and Banaji (1995) argue, it is "theoretically essential" (p. 5) that such indirect measures be used within the field of psychology to investigating people's implicit cognitions. This argument has been echoed by researchers interested in studying CSO cognition (e.g., Hanson \& Morton-Bourgon, 2004; Ward et al., 1997).

Almost two decades ago Stermac and Segal (1989) tried to lead sexual offender research away from the study of cognitive products and towards the experimental investigation of information processing operations. In a study looking at CSOs' interpretations of vignettes, Stermac and Segal compared 20 CSOs with 17 rapists, 35 male and female clinicians and 94 male and female community controls. All participants read 12 short descriptions of sexual encounters between an adult and child. Stermac and 
Segal varied the degree of sexual contact portrayed in each vignette across four levels ranging from touching to genital contact with ejaculation. In addition, the child's response to sexual contact was varied across three levels, from smiling, to passive/no response, to crying with resistance. Participants were asked whether the child enjoyed what happened, whether the child wanted it to happen, whether the child benefited from the experience and to what extent the child, and then the adult, was responsible. Answers were indicated using a five-point Likert scale. Compared to control groups, CSOs self-reported that the vignettes showed greater benefits and enjoyment for the child and less harm to the child. CSOs also reported more child desire and responsibility for the encounters and less adult responsibility. Also, CSOs needed to see a clearly negative response (i.e., crying) from the child before perceiving harm to the child.

Stermac and Segal (1989) concluded that CSOs may hold schemas or beliefs that lead them to misinterpret cues given by children during sexual encounters. The authors went on to suggest that these belief structures may form important treatment targets. While Stermac and Segal's conclusions may be correct, it should be noted that their methods allowed only a limited study of CSOs' information processing. Because participants were directly told what the child's reaction was, errors of perception couldn't be made. In other words, rather than measuring CSOs' misinterpretations of behaviour the authors asked them to rate the consequences of the behaviour (i.e., benefits and enjoyment). In this way, Stermac and Segal's vignette method echoed a questionnaire-based approach. It would have been easy for Stermac and Segal's participants to deliberately alter their responses. Controls, for instance, could have made sure their responses emphasised the harmful nature of sexual contact with children. Thus, the methodology may have allowed similar types of response biases that can occur using the questionnaire method of assessment described earlier. Of course, despite these limitations the study represented an important 
attempt to study CSOs' beliefs using fresh methods, and the findings may indeed indicate that CSOs interpreted the vignettes in line with distorted belief structures.

Despite Stermac and Segal's (1989) promising start, cognitive-experimental studies of CSO cognition were not readily taken up by researchers. Fortunately, this trend now appears to be changing, as recent years have yielded a handful of new studies in which CSO information processing is explored. For instance, three sets of researchers have recently used an experimental method called the Implicit Association Test to test the hypothesis that CSOs hold particular ITs. The Implicit Association Test (IAT) is a robust technique for measuring the strength of associations existing between concepts stored in long-term memory (see Greenwald, McGee, \& Schwartz, 1998; Swanson, Rudman, \& Greenwald, 2001). Put more simply, it measures how readily people associate words or images representing two contrasting concepts (e.g., young people and old people) with positive or negative meanings. Participants are presented with single words or images on a computer screen and asked to sort them into categories using a button press. One button is used to indicate two categories. For instance, a left button press might indicate a response meaning either slow or elderly, while a right button press might indicate quick or young. Thus, when the word crawl or an image of an elderly person appears on the computer screen, the left button should be pressed, and when the word sprint or an image of a young person appears the right button should be pressed. In a reversal of this task the left button is still associated with the word elderly, but it is also associated with the word quick, while the right button will now mean young and slow. The measure of interest within this example would be whether people are faster to categorise items when the words elderly and slow are associated with the same button, or when the words elderly and quick are associated. In all likelihood the former would occur, because when two concepts are highly associated in a participant's mind, they find it easier to sort words when the two 
associated concepts require the same response key than when they require different response keys.

Mihailides, Devilly, and Ward (2004) compared the IAT responses of 25 CSOs with 25 nonsexual offenders, 25 male university students, and 25 female university students. Mihailides et al. reasoned that if CSOs held a Children as Sexual Beings IT, they would be faster to link the concept of children with sexual words than nonsexual words, relative to controls. Likewise, if CSOs held Uncontrollable and Entitlement ITs they should be faster, relative to controls, to press buttons that link the concepts "losing control" and "mine" with sexual words than nonsexual words. It was found that compared to the three control groups, CSOs were significantly faster to link words congruent with the Children as Sexual Beings and Uncontrollable ITs than words incongruent with these theories. Compared to university controls CSOs were also faster to link Entitlementrelated and sexual concepts, although CSOs and offender controls could not be distinguished on the Entitlement IT. Mihailides et al. (2004) concluded that their results supported the hypothesis that CSOs hold ITs that influence their cognitive processing.

Gray, Brown, MacCulloch, Smith, and Snowden (2005) reported similar findings for stimuli designed to tap concepts that can be said to represent the Children as Sexual Beings IT. Like Mihailides et al., Gray et al. used the IAT paradigm to explore the degree to which child concepts, relative to adult concepts, were associated with sex. The responses of 18 CSOs and 60 nonsexual offenders were compared. CSOs' responses were slower when adult and sex words required use of the same response key than when child and sex word required the same response key. Nonsexual offenders demonstrated the opposite pattern.

Finally, Nunes, Firestone, and Baldwin (2007) also used the IAT to investigate the hypotheses that CSOs conceptualise children as being more pleasant, less powerful, and more sexually attractive than controls. The first two concepts would appear to tap into the 
Dangerous World IT, while the last concept taps the Children as Sexual Beings IT. Participants were 27 CSOs and 29 nonsexual offenders. As expected, the results indicated that CSO participants viewed children as more sexually attractive than adults. Strangely, however, nonsexual offenders did not differentiate between children and adults in terms of sexual attractiveness. Nunes et al. failed to comment on the unexpectedness of this finding, focussing instead on the fact that CSOs appeared to indicate a conception of children as being sexual agents. Also against predictions, the results indicated no differences between CSOs and nonsexual offenders regarding words that associated children with concepts of powerlessness or pleasantness.

In general, the above results have been interpreted as indicating that the IAT method can be used to indirectly and successfully measure the content held in CSOs' cognitive structures. Certainly, IAT techniques are an excellent tool for studying implicit cognition because they are relatively uncontaminated by dishonest or inaccurate responding (Asendorpf, Banse, \& Mücke, 2002; Greenwald et al., 2002; Greenwald \& Farnham, 2000). However, the conclusions some authors have drawn from the above studies are problematic as they do not support the hypothesis that CSOs hold schemas or beliefs that set them apart from nonsexual offending individuals per se. The studies do not demonstrate, unequivocally, that CSOs process and misinterpret information in ITconsistent ways; they simply show that CSOs hold stronger associations between certain concepts than others. CSOs may, for instance, hold stronger associations between children and sex concepts because they have discussed these associations more frequently throughout the conviction process or while interacting with prison psychologists. Similarly, semantic linkages between words pertaining to sex and losing control might be expected in men who are continuously confronted with the fact that a lack of self-control in relation to sexual matters has landed them in prison. Therefore, although IAT studies 
may reflect differences in the cognitive structures held by CSOs, their findings should be interpreted with caution.

In a piece of research that bears similarities to the above IAT studies, Kamphuis, De Ruiter, Jannsen and Spiering (2005) used a lexical decision task to see if semantic links existed between the concepts of sex and power in CSOs. As will be explained in detail in Chapter Four, the lexical decision task is an implicit measure of cognition that uses individuals' word processing speeds to analyse links between words and concepts held in long-term memory. The underlying rationale is that individuals make faster decisions about words when those words have been primed by prior presentation of a related word. Kamphuis et al. reasoned that if CSOs have strong links between sex words and power words, then presenting CSOs with words about sex should automatically prime words about power and thereby enable faster responses (compared to controls) to power words than other words, and vice versa. Although beliefs that sex and power are linked are not explicitly identified by Ward and Keenan (1999) as a type of IT, one could argue that people who hold a Dangerous World IT are concerned with power and may interpret sexual encounters as being about power and control. Kamphuis et al. found that compared to violent offender controls, CSOs were faster to respond to power words that had been preceded by sex words. Compared to student controls CSOs were not faster to respond to power words that had been preceded by sex words, although in their conclusions the authors interpreted this marginal result $(\mathrm{p}=0.51)$ as showing a significant difference. CSOs and student controls were also faster than violent offender controls to respond to sex words that had been preceded by power words. Thus this experiment provides some limited evidence that CSOs hold associations between the concepts of sex and power. However, as with the above IAT studies a potential semantic linkage between sex and power concepts among CSOs cannot be taken as evidence that CSOs hold offencesupportive beliefs. 
Another experimental study (Gannon, Wright, Beech \& Williams, 2006) recently utilized Stermac and Segal's (1989) idea of using written vignettes to investigate whether CSOs misinterpret social information. However, unlike Stermac and Segal, Gannon et al. did not ask participants to make judgments about what they had read, and instead asked participants to recall the material they had read. Gannon et al. reasoned that if CSOs hold offence-supportive beliefs they should interpret, and therefore recall, relevant material in a manner consistent with those beliefs. In the study 28 CSOs and 20 offender controls read a vignette describing a sexual offence against a child. Ten ambiguous statements that could be interpreted in an offence-supportive way were embedded within the vignette. That is, the statements could be interpreted in a manner consistent with belief content represented by four out of the five ITs identified by Ward (the Entitlement IT was not represented). For example, the child protagonist was described as putting her hand on the adult character's thigh, an action that could be interpreted in line with beliefs regarding Children as Sexual Beings). In a surprise recall task CSOs were asked to report what content they remembered from the vignette. Contrary to expectations, when each participant's free recall of the vignette was analysed, CSOs showed no difference in recall compared to other offenders; all participants misremembered the vignette, but did so in a way that was not offence-supportive. The results seemed to indicate that the CSOs' ITs were weak, absent, or inaccessible. Thus the findings did not align with Stermac and Segal's (1989) results, where CSOs apparently interpreted descriptions of adult-child sexual contact in offence-supportive ways. Gannon et al. used a more indirect measure of information processing (i.e., recall) than that used by Stermac and Segal (i.e., self-report). Therefore, Gannon et al.'s findings may indicate that participant responses in Stermac and Segal's study were influenced by other factors, such as participants' perceptions of what they ought to say. On the other hand, it is possible that offenders in Gannon et al.'s study censured their recollections so as to appear less distorted. However, the fact that few 
participants could articulate task aims in a follow-up questionnaire suggests that socially desirable responding was not an issue. In summary, the findings from Gannon et al.'s study are intriguing and certainly highlight the need for further controlled, empirical research into the nature of CSOs' beliefs. It is this research gap that will be addressed in this thesis.

\section{What's in a Measure?}

Throughout the past chapter three methods for measuring CSOs' offencesupportive beliefs have been reviewed. Two of these methods-conventional questionnaire and interview methods-try to measure beliefs by exploring the cognitive distortions that CSOs utter or endorse. Interview methods reliably find evidence of CSOs' cognitive distortions, and questionnaire methods frequently find that CSOs disagree with cognitive distortions less strongly than comparison groups do. Unfortunately, however, while measurement of these cognitive products may reveal the content of underlying beliefs, they may also reveal a range of other cognitive, affective, and contextually-driven phenomena.

Less-utilised is the cognitive-experimental method, wherein CSOs' cognitive processes are measured and compared to those of control groups. When designed well, experiments of this sort can bypass many of the conceptual issues and response biases associated with conventional methods of CSO belief measurement. Of the handful of cognitive experiments conducted with CSOs there has been mixed results. One study (Stermac \& Segal, 1989) found CSOs responded in a seemingly more distorted manner than controls. However, because this experiment minimised the opportunity for information processing errors to occur, and because it ultimately relied on participant selfreports, it is unclear what conclusions can be drawn from it. Three other studies have used the IAT method to investigate whether CSOs hold stronger associations between concepts that are thought to be linked within particular ITs. While these studies (along with a 
lexical decision task study) have found that CSOs have stronger semantic links than controls between particular concepts they do not actually demonstrate that CSOs process and misinterpret information in IT-consistent ways. Finally, a study by Gannon et al. (2006) used a recall experiment to explore whether CSOs process offence-relevant descriptions in a distorted way. No differences were found in the recollections of CSOs compared to controls. Although there is again some chance that CSOs in Gannon et al.'s study censured their responses, this final study raises questions about whether CSOs really do typically have belief structures that lead them to misinterpret important aspects of their social worlds.

In short, interviews consistently find that CSOs utter distorted statements that are often interpreted as reflecting faulty beliefs. Questionnaires are often able to differentiate between CSOs and others, which has also been interpreted as a sign that CSOs hold faulty beliefs. A handful of cognitive experiments have produced evidence that CSOs hold stronger associations between certain concepts, and one experiment has suggested that CSOs do not in fact hold beliefs that lead them to misinterpret information. If these three different method types all measure the prevalence and nature of CSOs' offence-supportive beliefs, one would expect their findings to be more consistent with each other. That is, interviews, questionnaires and cognitive experiments should show some agreement over the frequency with which offence-supportive beliefs occur among CSOs and the types of beliefs that individual CSOs are shown to endorse on each measure should roughly align.

So to what extent $d o$ interview, questionnaire and experimental measures of offence-supportive beliefs show agreement regarding the frequency and nature of CSO beliefs? Amazingly, this question is unanswerable, because of the fact that it has gone virtually unexplored. In fact, a review of the literature suggests that only one published study has even approached the topic of multimethod comparison. Neidigh and Krop (1992) gave the question some notice by counting the number of cognitive distortions that 
CSOs endorsed in an interview and comparing that with the number of items they endorsed on a questionnaire (i.e., the ABCS). They found that, on average, twice as many cognitive distortions were counted per CSO using the interview technique. As Neidigh and Krop stated, this frequency difference suggests that interviews and questionnaires might not be measuring the same phenomena. As noted earlier, interviews might elicit more distortions because the underlying phenomena being revealed (i.e., distorted statements) are social desirability processes, rather than beliefs. On the other hand both measures might be eliciting beliefs, but in interviews they are more evident because, for instance, CSOs' belief structures are maximally activated, or because CSOs underreport agreement with questionnaire items. To shed light on this issue it would have been helpful if Neidigh and Krop had also noted whether there was agreement about the types of distortions that CSOs endorsed across the two measures. This may have given some clue as to whether both measures were in fact tapping similar belief content. Unfortunately this information was not provided by Neidigh and Krop.

As far as agreement between cognitive-experimental and conventional measures of CSO belief is concerned, no comparisons appear to be available. Looking at more general areas of enquiry, Fazio and Olson (2003) have noted that when measuring socially undesirable constructs, low correlations are often found between implicit (e.g., cognitiveexperimental) methods and explicit (e.g., interview and questionnaire) methods. Fazio and Olson explain that this might be because people can more readily fake good on explicit self-report measures. Again, reviewing the types of beliefs that CSOs appear to endorse on implicit versus explicit measures might give some indication of whether the differing methods overlap to some extent in the types of constructs that they measure. If implicit and explicit methods both measure the content of belief structures then it should be expected that at least some agreement will emerge across implicit and explicit measures in terms of the types of beliefs each CSO endorses. In other words, when CSOs do appear to 
endorse beliefs in interview, questionnaire and experimental measures, even if the level of belief endorsement differs, the types of beliefs that they endorse should show some agreement. In the final study reported in this thesis the need for research utilising multimethod assessment of CSOs' beliefs will at last be addressed. 


\section{Research Agenda}

A primary aim of this research is to use implicit measures of cognition to better understand the belief structures of CSOs and explore how they relate to cognitive distortions. The previous chapters have outlined issues and controversies surrounding the measurement of CSOs' offence-supportive beliefs. In short, current measures have made it difficult to tell whether CSOs do in fact hold beliefs that lead them to endorse offencerelevant statements and process information in offence-relevant ways. This thesis will attempt to extend the literature through the use of controlled experimental techniques adapted from the field of cognitive science. The techniques used will curtail participants' ability to censure their responses. In addition, participants will not be required to perform the difficult (or even impossible) task of introspectively and retrospectively reflecting on cognitive structures and processes active at the time of their offending. As a way of shedding further light on the phenomena being measured by implicit and self-report measures of CSOs' beliefs, this research will also investigate whether correlations can be found between interview, questionnaire and experimental measures.

The first study, reported in Chapter Four, describes the unique use of an adapted version of a lexical decision task. Lexical decision tasks have previously been used to study belief content in individuals by measuring their response times to particular words. In Study One the lexical decision task is used to seek support for the hypothesis that CSOs hold beliefs containing IT-consistent themes which set them apart from offender and community-based controls.

In Chapter Five a second study is described in which a memory-based task previously used to study information processing in violent men is applied to the study of CSOs' belief structures. CSOs and offender controls are compared in their recall for ambiguous information tapping the Children as Sexual Beings IT. This study will also 
make use of visual primes, which will be presented in an attempt to activate offencesupportive beliefs.

Finally, Study Three in Chapter Six takes an in-depth exploration of the types of beliefs that CSOs are classified as holding according to three measurement methods. CSOs and offender controls are interviewed and their offence explanations coded for the presence of IT-congruent themes. Next, both CSOs and offender controls complete a different type of cognitive experiment that has been used in other fields to study beliefs and schemas, and their responses are compared. CSOs' and offender controls' answers on a questionnaire measure of offence-supportive beliefs are also compared. The types of beliefs that CSOs endorse according to each of these measures are investigated and an analysis of the level of agreement between the measures is conducted.

\section{A general note on the recruitment of participants}

The three methods used to investigate CSOs' belief structure in this thesis were implemented within the space of two years across three prisons. Therefore, there was overlap between participants, with some offender participants taking part in two or three of the following studies. Rates of overlap are discussed within Chapters Five and Six.

In all three studies offender participants were not recompensed in any way for their participation, although chocolate biscuits were offered for consumption during each research session. In Study One (the only study that used community-based controls) a $\$ 20$ music voucher was given to community participants.

\section{A general note on written material provided to participants}

Across the three studies conducted in this thesis a large number of documents were distributed to participants. In each study recruitment sheets, information sheets, consent forms, debriefing sheets, and feedback forms were distributed, and sometimes these documents contained variations according to the participant group for which they were written (e.g., community-based controls and offenders received different recruitment 
flyers that reflected their different circumstances). To conserve space not all of these documents have been included in the Appendices. Instead, one example of each type of participant document can be found in Appendices Eleven to Fifteen. 


\section{Chapter Four}

\section{An Experimental Study of Offence-Supportive Beliefs: The Lexical}

\section{Decision Task}

\section{Introduction}

The previous chapter described the existing over-reliance on self-report methods to explore CSOs' offence supportive beliefs. There is a clear gap in the research literature that calls for the use of controlled, experimental studies to investigate CSOs' belief structures. This chapter will introduce the lexical decision task, a technique that allows for the indirect measurement of cognitive structures and their contents.

\section{Study One: The Lexical Decision Task}

As mentioned in the preceding pages, cognitive structures can aid cognitive processing. For instance, when cognitive structures such as beliefs and schemas are active they enable faster processing of information related to the contents of those structures and can enhance recall of relevant information (e.g., Fiske \& Taylor, 1991; Rudman, Greenwald, Mellot, \& Schwartz, 1999). Conversely, information will take longer to process if it is incongruent with an active schema or belief. The lexical decision task is an experimental paradigm that exploits the fact that cognitive structures can facilitate or inhibit information processing, in order to gain glimpses of the nature and content of people's underlying cognitions.

The lexical decision task (LDT) was first performed by Meyer and Schvaneveldt (1971), who asked participants to indicate whether two simultaneously presented words were real words or nonwords. Results showed that participants were faster to respond when the words were semantically related, (e.g., bread and butter) than when they were unrelated (e.g., doctor and butter). While differing versions of the LDT have since been performed, the overarching results are the same: people are quicker to identify words that 
follow contextual primes (i.e., a related word or concept) than words that have followed non-related primes (Neely, 1991). For example, when a participant sees the sentence stem John ate the... they will be faster to recognise the target word food than the word foot (Forster, 1981). Research has indicated that this so-called "sentence context effect" occurs only if the target word is the expected completion of the sentence stem (Fishler \& Bloom, 1979). In other words, participants will respond faster to the target word only if they cognise that it is a likely ending to the sentence. This finding makes sense in light of the fact that readers use the content of their individual cognitive structures to anticipate text content (Duffy, 1986; Fincher-Kiefer, 1992; Fitzgerald, 1995) and to spend less time processing the ends of sentences (Sharkey \& Sharkey, 1987).

Researchers have successfully used these findings as a means for exploring the content of people's beliefs (e.g., Baldwin, Fehr, Keedian, Seidel, \& Thomson, 1993; Kay \& Jost, 2003; Mikulincer, 1998; Mikulincer, Birnbaum, Woddis, \& Nachmias, 2000). For instance, Baldwin et al. used a version of the lexical decision task to explore the beliefs that people with different attachment styles have about others. Baldwin et al. compared the way that groups of individuals with different attachment styles processed sentences that presented alternative interpersonal outcomes. After identifying each participant as either secure, avoidant, or anxious-ambivalent, the authors asked participants to read context sentences followed by target words that were designed to reveal their interpersonal expectations. For example, the context sentence if I try to get close to my partner then my partner will...might be followed by the positive outcome word accept or the negative outcome word reject. Each context sentence was briefly presented on a computer screen to participants, and was immediately followed by either a negative outcome word, a positive outcome word, or a nonword. Participants were simply instructed to indicate (by pressing a button) whether each outcome word they viewed was a real or a made-up word. Of interest was the time participants took to make each decision. Baldwin et al. reasoned that 
securely-attached participants would hold positive expectations about their partners in terms of three domains (trust, closeness and dependency). Therefore, beliefs about the trustworthiness, closeness, and dependability of partners (which ought to have been activated by context sentences) should have lead securely attached participants to respond more quickly to positive outcome words within the three domains. Conversely, insecurelyattached participants should have held negative expectations about their partners and therefore should have been faster to respond to negative outcome words. This pattern of result is exactly what was found.

In 1998 Mikulincer replicated Baldwin et al's (1993) result after using a lexical decision task to compare people with different attachment styles in terms of their beliefs about how others are likely to react to anger. In another study, Kay and Jost (2003) investigated whether "just world beliefs" (i.e., beliefs that the world is fair and people get what they deserve) can influence the speed of individuals' lexical decisions about justicerelated words. The authors found that when they threatened (and thereby activated) individuals' just world beliefs, those individuals were faster to respond to justice-related words than neutral words, and were faster than control participants (whose beliefs hadn't been activated) to respond to justice-related words.

The current study adopts Baldwin et al.'s approach in order to investigate the beliefs of CSOs, offender controls, and community-based controls. Participants are presented with a series of context sentences that relate to Ward and Keenan's (1999) five ITs described in Chapter Two. Each context sentence is followed by a letter string that participants quickly identify as a word or a nonword. Target words are either IT-consistent (i.e., complete sentences in a way consistent with IT-based propositions), or ITinconsistent (i.e., do not complete sentences in a way consistent with IT-based propositions). It is hypothesised that CSOs will interpret context sentences in a manner 
consistent with offence-supportive schemas and thus, relative to controls, will be quicker to respond to IT-consistent than IT-inconsistent words.

\section{METHOD}

\section{Participants}

One hundred men took part in this study: 32 CSOs, 37 offender controls, and 31 community-based controls. CSOs and offender controls were recruited from three New Zealand Department of Corrections prisons based in the lower North Island. All offenders were recruited by unit managers, who distributed a recruitment flyer to prisoners inviting them to put take part in a "word decision" study. Because the current author did not directly distribute fliers to prisoners it is unclear what proportion of prisoners who were asked to take part in the study agreed to participate.

Community controls were recruited by emailing recruitment flyers to staff at the Wellington Branches of The Samaritans and Youthline (two local volunteer helpline organisations) and to a group of approximately 40 staff members within a large New Zealand financial institution. Recruitments flyers for all participants stated that participants should be able to read reasonably well (i.e., they should be able to read the recruitment flyer with ease and have had the necessary skills to pass a year-10 secondary school English paper such as New Zealand Fifth Form Certificate English or its equivalent). A total of 115 men completed the experiment (41 child sexual offenders, 43 offender controls, and 31 community controls). However, nine CSOs and six offender controls were dropped from the study because on the lexical decision task they made more than six errors out of 45 trials; an error rate of $13 \%$. It should be noted that previous LDT studies that have used the design on which this study is based have not addressed the issue of how an appropriate cutoff rate is best determined. For instance, Baldwin et al. (1993) removed (without explanation) two participants who had $26 \%$ and $36 \%$ error rates, while Mikulincer (1998) and Mikulincer et al. (2000) simply mention that across all participant 
between $2.3 \%$ and $4.8 \%$ of responses were errors. Surprisingly, Kay and Jost (2003) do not address the issue of error rates at all. Thus, it appears that there is no agreed-upon method for determining error rate cutoffs. In the current study the rate was determined by selecting participants whose error rates placed them as outliers compared to all other participants combined. Selection criteria and demographic details for each participant group follows. Only details for those men included in the study are reported.

\section{Group selection criteria and demographic details}

CSOs

CSOs were men who at the time of testing were incarcerated for sexually abusing a person under the age of 16. CSOs were aged from 21 to 77 years $(M=48.47, S D=15.07)$ and their victims' ages ranged from 2 to 15 years $(M=10.13, S D=3.51)$. Although an initial aim was to recruit only untreated, extrafamilial CSOs, the inclusion criteria had to be relaxed slightly to meet sample size requirements. According to current and historic conviction records, 20 CSOs were extrafamilial offenders (i.e., CSOs who had molested at least one child who was not a family member), while 12 CSOs were intrafamilial offenders (i.e., CSO who had molested only biological or non-biological family members). Three of the extrafamilial and none of the intrafamilial offenders had received cognitivebehavioural therapy to reconstruct distorted offence-related cognitions. The three treated CSOs had all committed at least one sexual assault against a child since receiving therapy. The mean Static-99 score for all CSO participants indicated a medium recidivism risk level $(M=3.06, S D=2.38)$, as defined by Phenix, Hanson, and Thornton (2000). The number of years that CSOs had spent in formal education ranged from nine to 11 years ( $M$ $=10.03, S D=0.59$ ). Five CSOs identified themselves as Māori, 21 as European, and six as "other". Fourteen CSOs had committed a violent offence at some stage in their offending history, with ten of these having committed two or more violent offences. 
Offender controls (OCs)

OCs were men who were serving a prison sentence for one or more offences, but who had never been convicted of a sexual offence against a person under the age of 16 . Of the OCs, four men had a conviction for a sexual offence against a person over 16 listed in their conviction history. OCs were aged from 18 to 69 years $(M=33.68, S D=10.82)$. The number of years that OC participants had spent in formal education ranged from nine to 11 years $(M=9.60, S D=0.64)$. Eighteen OCs identified themselves as Māori, 16 as European, and three as "other". Twenty-eight OCs had a violent offence recorded in their conviction histories (index offences included), with 26 of these having committed two or more violent offences.

\section{Community controls (CCs)}

Community controls were males living in Wellington, NZ, who had never served a prison sentence or been convicted for a sexual offence. CCs were aged from 21 to 59 years $(M=37.42, S D=11.11)$. The number of years that CCs had spent in formal education ranged from nine to 11 years $(M=10.03, S D=0.59)$. Four CCs identified themselves as Māori, 22 as European, and 5 as "other".

Characteristics of the participant groups are displayed in Table 4.1. The groups differed significantly on age, $F(2,97)=12.85, p<.001$, with CSOs having a higher mean age than $\mathrm{OCs}(M=48.47, S D=15.07$ versus $M=33.68, S D=10.82)$. CCs $(M=37.42$, $S D=11.11$ ) did not differ in age from either offender group. The groups also differed significantly on number of years spent in formal education, $F(2,97)=6.20, p=.003$, with CSOs $(M=10.03, S D=0.59)$ and CCs $(M=10.03, S D=0.55)$ having spent more years in formal education than OCs $(M=9.59, S D=0.64)$. A chi-square test of association also confirmed that between-group differences in ethnicity existed, $\chi 2(4, N=100)=14.32, p$ $=.006$, Cramer's $\mathrm{V}=.268$, with OCs having a significant difference in ethnic mix compared to CSOs, $\chi 2(2, N=69)=8.71, p=.013$, Cramer's $\mathrm{V}=.355$ and CCs, $\chi 2(2, N$ 
$=68)=9.90, p=.007$, Cramer's $\mathrm{V}=.382$. Significant differences also existed in total number of violent convictions recorded in CSOs' and OCs' prison records, $t(67)=3.08, p$ $=.003$, with OCs $(M=4.08, S D=4.03)$ having significantly more mean violent convictions per participant than CSOs $(M=1.53, S D=0.45)$.

$\underline{\text { Table } 4.1}$

Demographic details for participant groups: Study 1

\begin{tabular}{|c|c|c|c|}
\hline & $\begin{array}{l}\text { Child Sex } \\
\text { Offenders }\end{array}$ & $\begin{array}{l}\text { Offender } \\
\text { Controls }\end{array}$ & $\begin{array}{c}\text { Community } \\
\text { Controls }\end{array}$ \\
\hline & $n=32$ & $n=37$ & $n=31$ \\
\hline \multicolumn{4}{|l|}{ Age (years) } \\
\hline Mean & 48.47 & 33.68 & $37.42 * * *$ \\
\hline$S D$ & 15.07 & 10.82 & 11.11 \\
\hline \multicolumn{4}{|c|}{ Years in education } \\
\hline Mean & 10.03 & 9.59 & $10.03 * *$ \\
\hline$S D$ & 0.59 & 0.64 & 0.55 \\
\hline \multicolumn{4}{|l|}{ Ethnicity $(n)$} \\
\hline M aori & 5 & 18 & $4 * *$ \\
\hline European & 21 & 16 & $22 * *$ \\
\hline Other & 6 & 3 & $5 * *$ \\
\hline \multicolumn{4}{|c|}{ Violent Convictions (n) } \\
\hline Mean & 1.53 & $4.08 * *$ & - \\
\hline$S D$ & 2.55 & 4.03 & - \\
\hline \multicolumn{4}{|l|}{ CSO type $(n)$} \\
\hline Intrafam ilial & 12.00 & - & - \\
\hline Extrafamilial & 20 & - & - \\
\hline \multicolumn{4}{|c|}{ Child vicitms (n) } \\
\hline Mean & 3.41 & - & - \\
\hline$S D$ & 3.15 & - & - \\
\hline \multicolumn{4}{|c|}{ Victim age (years) } \\
\hline Mean & 10.13 & - & - \\
\hline$S D$ & 3.51 & - & - \\
\hline \multicolumn{4}{|c|}{ ST A T IC-99 Score } \\
\hline Mean & 3.06 & - & - \\
\hline$S D$ & 2.38 & - & - \\
\hline
\end{tabular}




\section{Ethical Considerations}

Victoria University of Wellington and New Zealand Department of Corrections approved all procedures and materials used in this study. Before testing, participants read an information sheet outlining the details of the study and their participant rights (which accorded with NZPS Code of Ethics). The information sheet warned participants that during the experiment written material would be presented that referred to criminal activity, and some of this material would "link children and sex". Participants were told that they would need to provide some background information about themselves, and offender participants were told that an experimenter would check their prison records after testing was complete. All participants signed a consent form before taking part in the experiment. They were also given the opportunity to request a combined debriefing and results summary sheet once results had been collated.

\section{Design}

The experimental design was based on the LDT method used by Baldwin et al. (1993). A computer task was set up so that participants were presented with 45 incomplete sentences (word stems), each of which was followed by a word or a nonword. Each word stem related to one of the five belief clusters on which Ward and Keenan's (1999) ITs are based. Thirty words and 15 nonwords followed the 45 word stems. Fifteen of the thirty words completed the sentence in a way that was offence-supportive (i.e., consistent with IT-related beliefs), while the other fifteen words completed the sentence in a non-offence supportive manner (i.e., inconsistent with IT-related beliefs). 


\section{Materials}

It was possible that reading speed differences between participants might have a confounding effect on LDT responses. Therefore, before the experiment commenced participants completed a reading speed task that would allow adjustment of LDT responses according to individual differences. Ten simple-structure sentences and ten complex-structure sentences (rated on structure complexity by Fischler \& Bloom, 1979) were presented to participants via the Superlab programme (see Appendix One). Participants were asked to press any button on the response pad to indicate that they had finished reading each sentence.

Forty-five incomplete word stems were created, and to each word stem three letter strings (one offence-supportive completion word, one non offence-supportive completion word, and one nonword) were assigned. Nonwords were generated by taking common verbs (e.g., dribble, dance) and changing one letter (e.g., kribble, wance). Nonwords were matched for number of characters with real words. An ANOVA confirmed that target word length did not differ significantly across completion word type (offence-supportive, non offence-supportive, and nonword), $F(2,132)=0.18, p=.837$. A t-test confirmed that offence-supportive and non offence-supportive target words did not differ according to ranked frequency of use in New Zealand English, $t(88)=-1.19, p=.236$.

Below is an example word stem and three letter strings that were assigned to it (for a full list of word stems and letter strings used in this experiment refer to Appendix Two):

\section{Word Stem}

Having sex with children won't do them any

\section{Letter String}

HARM (Offence-supportive)

GOOD (Non Offence-supportive)

KNID (Nonword)

Three versions of the LDT were created and 45 of 135 possible word stem/letter string combinations were assigned to each version (15 Offence-supportive, 15 Non 
Offence-supportive, 15 nonwords). Participants were randomly assigned to Versions One, Two, or Three of the LDT ${ }^{2}$. Within each LDT version, the five IT categories identified by Ward and Keenan (1998) were represented evenly among the 45 sentences that were presented (i.e. nine sentences representing each IT were shown). No participant saw the same word stem or letter string twice.

\section{Apparatus}

The reading task and LDT were programmed using a Superlab package. The program was run on a 12 inch colour monitor screen, resolution $=1024 \times 768$ pixels from a Dell Pentium Latitude D600 laptop computer. Written instructions and stimuli were centred on a white screen in bold black Times New Roman text (font size 40). Participants made their responses on a Cedrus Model RB-730 response pad that held seven response keys. The two endmost keys had a coloured covering (one yellow and one green) while the keys in between were white. Two labels (reading "made-up word" and "real word") were placed on the response pad above the two coloured keys; placement of labels was counterbalanced across participants.

The Superlab program controlled the random presentation of 20 sentences in the reading task and recorded (in milliseconds) the time that elapsed between each button press the participant made to view a new sentence. The programme also controlled the random presentation of word stems and letter strings in the LDT, and recorded the response time (RT) and type of response (correct / incorrect) made. Each word stem was presented to participants individually for $3000 \mathrm{~ms}^{3}$. Immediately following presentation of each incomplete sentence, a letter string (i.e., a word or a non word) appeared on the

\footnotetext{
2 ANOVAs confirmed that participant groups in the three LDT versions did not significantly differ in terms of age, years of education, and reaction times to offence-supportive words, nonoffence-supportive words or nonwords. A Chisquare test found no differences in ethnicity.

${ }^{3}$ Incomplete sentence presentation duration was based on feedback from five independent community-based raters. On completion of the experiment all participants were asked to rate sentence presentation on a 5-point Likert Scale ranging from 0 (Much too quickly) to 4 (Much too slowly). Mean rating was 2.11, indicating that, on average, participants felt incomplete sentences were presented "at the right speed".
} 
screen for $2000 \mathrm{~ms}$. To encourage rapid responding, the computer emitted a loud whistling sound if a participant did not register their response to the letter string within $2000 \mathrm{~ms}$.

\section{Procedure}

Participants were tested individually, in a single session. Each testing session took approximately 30 minutes per participant. After giving written consent, all participants were asked to give some basic demographic information about themselves such as age, ethnicity, and years of formal education. Offender participants were asked about the nature and number of their crimes. CSOs were also asked to provide details of their sexual offences (e.g., age and number of victims, relationship to victims) and to answer other questions that would allow calculation of their Static-99 scores. Next, participants were verbally instructed on how to complete the reading test. They were told to read at a normal, comfortable reading speed, and to press any button on the response pad once they had read the full sentence. Unfortunately, despite instructions participants were often observed not paying attention during this reading task and many also attempted to talk during the task. Because these reading speed measures appeared to be an unreliable measure they were not used in the final analysis.

Next, participants were given verbal instructions on how to complete the LDT and were taken through a practice phase. The experimenter watched as each participant completed the practice run. If any participant appeared to be struggling with the task the experimenter would take the participant's place and have them watch as the experimenter completed the practice run. The participant would then complete the practice phase again.

Upon completion of a satisfactory practice run, participants were told to follow the same procedure during the experimental phase that they did during the practice phase, paying careful attention to each word stem. All participants were again warned that they would see material during the task that they may find distasteful, but to try not to be distracted by this. It was stressed that their task was not to make a value judgment about 
the appropriateness of completion words, but rather to simply indicate whether they were viewing a word or nonword. When they were ready to start the experimental phase participants pressed any key on their response pad. To make participants feel comfortable during the task, the experimenter stayed in the room but faced away from the computer screen and read some papers until the participant indicated that they had finished.

\section{RESULTS}

Results were analysed to see whether they confirmed the hypothesis that CSOs alone would respond in a way that indicated they held schemas containing offencesupportive content. CSOs', OCs', and CCs' reaction times were compared to see whether CSOs were faster to identify words that completed sentences in an IT-consistent way (i.e., offence-supportive words) than words that did not complete sentences in an IT-consistent way (i.e., nonoffence-supportive words).

To investigate, each participant's reaction times (RTs) in milliseconds (ms) were summed and averaged to produce mean reaction times within 12 variables. The 12 variables were: All offence-supportive word RTs; All nonoffence-supportive word RTs; Offence-supportive word RTs for each of the five individual IT categories; Non offencesupportive word RTs for each of the five individual IT categories.

To measure each participant's basic speed of responding, mean reaction times to the 15 nonwords were also calculated. Because nonwords are devoid of semantic meaning they could not be primed by sentence stem content; mean nonword RTs therefore served as a covariate measure of participant cognitive processing and motor response speed. It should be noted here that although age and years of formal education significantly differentiated the groups (see Method), neither of these factors were significant covariates and so are not reported in the following analyses. 


\section{Data preparation}

Only RTs from correct responses were analysed ${ }^{4}$. RT data generally contains outlying data points that represent factors unrelated to the hypotheses (e.g., attentional lapses and unintentional motor responses). Thus, it is important to separate these outliers from data that reflect reactions arising from the cognitive mechanisms under investigation (Ratcliffe, 1993). In accordance with previous research (e.g., Cumming, Graham \& Patterson, 2006; Holden, Kroner, Fekken, \& Popham, 1992; Ratcliffe, 1993; Welford, 1980), measures were taken to adjust for such outliers. Mean RTs to nonwords and to each of the 12 variables described above were Windsorised (Barnett \& Lewis, 1978). That is, extremely high RT scores (i.e., RTs more than two standard deviations above the group mean) were assigned the next lowest RT for that group and especially slow RT scores (i.e., RTs more than two standard deviations below the group mean) were assigned the next highest RT for that group. This adjustment ensured that all RTs were included in the data set while mitigating for the impact of potentially spurious responses.

Table 4.2 lists the three participant groups' mean Windsorised reaction times to the nonwords and the 12 variables listed above.

\footnotetext{
${ }^{4} 99 \%$ of all responses were correct.
} 
Table 4.2.

Participant groups' mean reaction times (in milliseconds) to LDT words and nonwords

\begin{tabular}{|c|c|c|c|c|c|c|}
\hline & \multicolumn{2}{|c|}{$\mathrm{CSO}$} & \multicolumn{2}{|c|}{$\mathrm{OC}$} & \multicolumn{2}{|c|}{$\mathrm{CC}$} \\
\hline & Mean & SD & Mean & SD & Mean & SD \\
\hline All Nonwords & 1025.18 & 154.54 & 1040.24 & 167.88 & 824.14 & 150.76 \\
\hline \multicolumn{7}{|l|}{ Offence- } \\
\hline \multicolumn{7}{|l|}{ Supportive } \\
\hline All ITs combined & 962.92 & 187.68 & 942.51 & 164.05 & 773.46 & 148.62 \\
\hline Children as & 990.92 & 192.00 & 958.48 & 189.26 & 823.54 & 174.99 \\
\hline \multicolumn{7}{|l|}{ sexual beings } \\
\hline Dangerous world & 871.54 & 161.92 & 871.99 & 155.45 & 725.45 & 141.25 \\
\hline Entitlement & 956.69 & 233.41 & 909.92 & 203.58 & 757.18 & 197.11 \\
\hline Nature of harm & 1014.50 & 295.97 & 941.62 & 193.69 & 757.34 & 167.13 \\
\hline Uncontrollability & 956.93 & 215.38 & 1014.80 & 255.98 & 794.30 & 165.78 \\
\hline \multicolumn{7}{|l|}{ Non Offence- } \\
\hline \multicolumn{7}{|l|}{ Supportive } \\
\hline All ITs combined & 929.13 & 173.68 & 872.52 & 110.52 & 778.50 & 133.88 \\
\hline Children as & 938.41 & 210.17 & 920.59 & 149.61 & 752.25 & 138.64 \\
\hline sexual beings & & & & & & \\
\hline Dangerous world & 857.55 & 206.38 & 788.27 & 146.75 & 727.65 & 162.50 \\
\hline Entitlement & 946.51 & 174.12 & 887.26 & 150.11 & 806.59 & 150.55 \\
\hline Nature of harm & 930.47 & 213.23 & 860.67 & 156.13 & 803.20 & 167.59 \\
\hline Uncontrollability & 974.14 & 188.31 & 887.93 & 155.05 & 787.55 & 148.65 \\
\hline
\end{tabular}




\section{Main analysis of response times}

To calculate each participant's shift in reaction time between the two word types, mean RTs to all offence-supportive words were subtracted from mean RTs to all nonoffence-supportive words. This generated an overall mean difference in RTs, with positive scores indicating faster responding to offence-supportive content relative to nonoffence supportive content (i.e., responses are offence-supportive). Negative scores, on the other hand indicate faster responding to non offence-supportive content relative to offence supportive content (i.e., responses are non offence-supportive).

To investigate the effects of group (CSO, OC, CC) and word type (offencesupportive, nonoffence-supportive) on reaction time, an ANCOVA was carried out with mean difference in RTs as the dependent variable and group as the fixed factor. That is, an ANCOVA was conducted that compared the groups on their responses to offencesupportive words overall versus nonoffence-supportive words overall. Nonword RTs were identified as a significant covariate and were added to control for individual differences in generalised processing and response speeds. The results of the ANCOVA were surprising, as they demonstrated no significant interaction, $F(2,96) 2.08, p=.131$, partial eta squared $=.041$. In other words, against predictions CSOs did not respond faster to offencesupportive words relative to nonoffence-supportive words when compared to OCs and CCs (see Figure 4.1.). Bonferroni post-hoc tests revealed that no group (CSO, OC or CC) could be statistically differentiated from another. Interestingly, these results held regardless of CSO offence characteristics. In other words, when the ANCOVA was run with only intrafamilial CSOs' responses or only extrafamilial CSOs' responses included in the data, the above pattern of results was replicated. In addition, CSOs' Static-99 scores were not found to correlate with their overall mean difference in RTs. To check that the above findings were unaffected by the fact that four OC participants had sexually offended against adults, the above ANCOVA was rerun with their data removed from the analysis. 
Again, the pattern of results remained unchanged. Contrary to predictions, shifts in RTs to offence-supportive and nonoffence-supportive words ran in the nonoffence-supportive direction for CSOs $(M=-25.80 \mathrm{~ms})$, as well as OCs $(M=-59.87 \mathrm{~ms})$, and CCs $(M=-$ $15.28 \mathrm{~ms})$. Hence, all three groups demonstrated a pattern of responding seeming to indicate that they did not hold offence-supportive schemas.

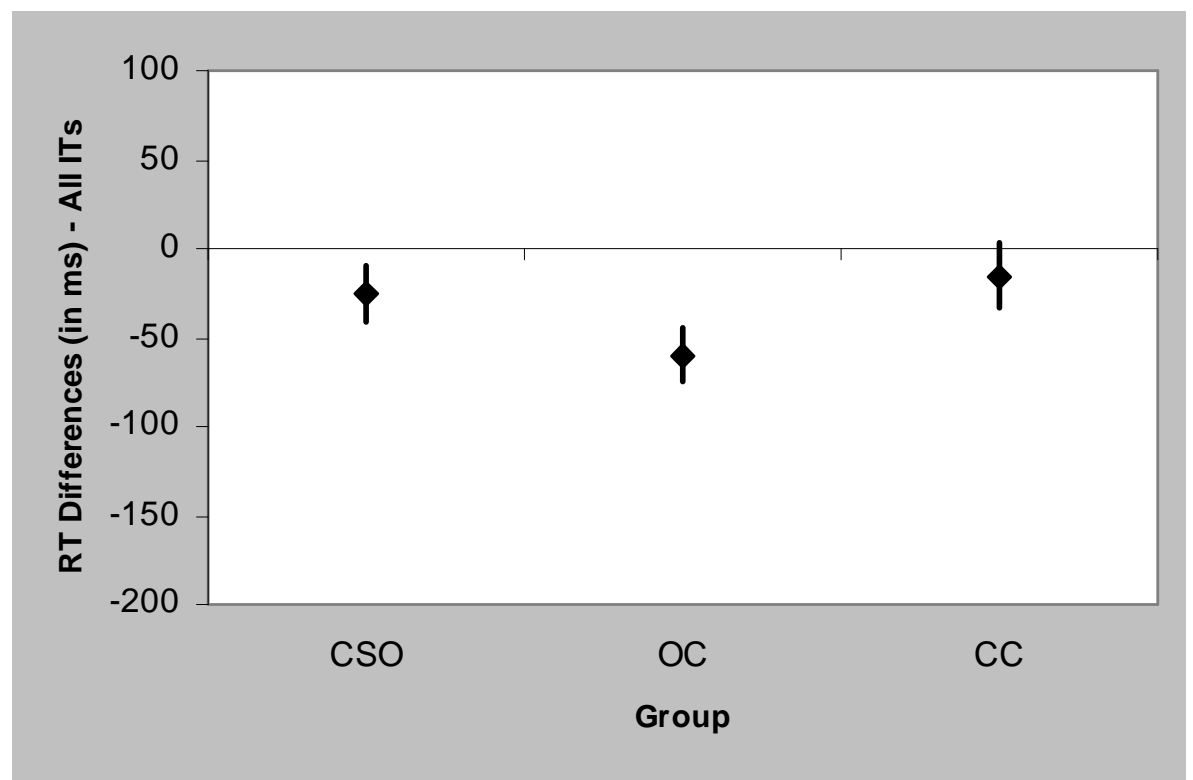

Figure 4.1. Mean Differences (+/- SEM) in Offence-supportive and NonoffenceSupportive Reaction Times to All Implicit Theories

While overall responses did not indicate that CSOs were interpreting information in an offence-supportive way, more detailed analysis might reveal that CSOs appear more distorted within certain IT categories. To investigate whether this was the case, a mean difference in offence-supportive and non offence-supportive RT responses for each of the following categories of items was generated: Children as Sexual Beings, Dangerous World, Entitlement, Nature of Harm, and Uncontrollable. 
For each of these five ITs an ANCOVA was run to examine the effects of group (CSO, OC, CC) and word type (offence-supportive, nonoffence-supportive) on mean reaction time. For each ANCOVA, mean difference in RTs was the dependent variable, group was the fixed factor, and mean nonword RTs were the covariate (although the pattern of results for all analyses described below pertained when mean nonword RTs were not added as a covariate, with the uninterpretable exception that CCs obtained more positive scores than CSOs for Nature of Harm, and CSOs in turn obtained more positive scores than OCs). Because the analysis involved three t-tests, alpha was set at .01 to compensate for the associated inflation of type 1 error.

The Children as Sexual Beings ANCOVA did not reveal a significant interaction between group, word type, and mean reaction time, $F(2,96) 0.15, p=.862$, partial eta squared $=.003$. Likewise, significant interactions were not found for Dangerous World, $F(2,96) 2.08, p=.131$, partial eta squared $=.041$, Entitlement, $F(2,96) 0.08, p=.927$, partial eta squared $=.002$, and Nature of Harm, $F(2,96) 1.67, \mathrm{p}=.194$, partial eta squared $=.034$. However, the ANCOVA examining Uncontrollable did demonstrate a significant interaction, $F(2,96) 4.33, p=.016$, partial eta squared $=.083$. Bonferroni post-hoc tests revealed that CSOs' RT shift $(M=20.35 \mathrm{~ms})$ was significantly different $(p=.005)$ to OCs' $(M=-122.90 \mathrm{~ms})$. Although CSOs' RT shift was not significantly different $(p=.552)$ from that of CCs $(M=-14.75 \mathrm{~ms})$, it should be noted that CSOs demonstrated a positive shift in RTs, indicating an offence-supportive response pattern, while OCs and CCs demonstrated a negative (nonoffence-supportive) shift (see Figure 4.2.). Note that the standard error of the mean for Uncontrollable is larger than for all ITs combined. This pattern was found for each of the five ITs when they were considered in isolation and reflects the fact that a smaller sample size (i.e., number of scores) comprised mean RT differences within each IT than for all ITs combined. 


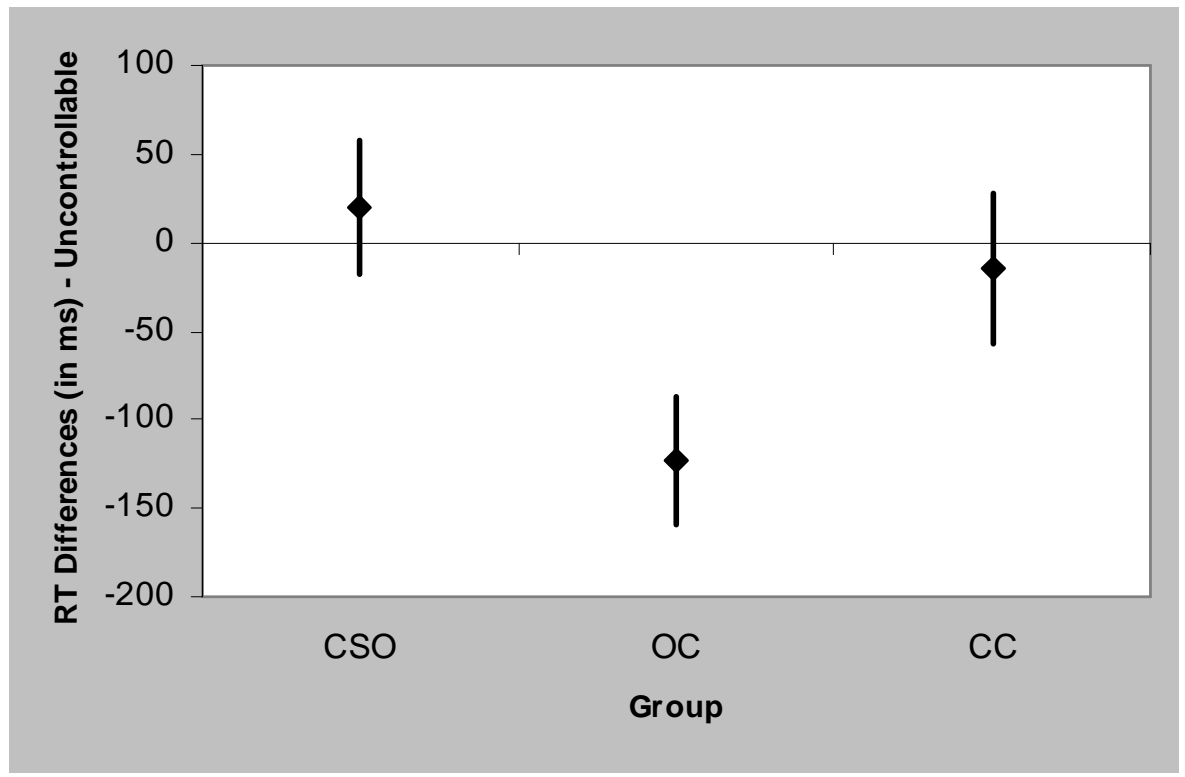

Figure 4.2. Mean Differences (+/- SEM) in Offence-supportive and NonoffenceSupportive Reaction Times to Uncontrollable

Because significant ethnic group differences existed between groups (i.e., Māori comprised $49 \%$ of the OC sample, compared to only $16 \%$ of the CSO and $13 \%$ of the CC samples), one-way ANOVAs were run to see whether all (CSO, OC, and CC) Māori participants responded differently to each of the ITs than all Europeans or all those in the "Other" ethnic category. For each ANOVA, mean difference in RT was the dependent variable, and ethnicity (Māori, European, Other) was the fixed factor. The three ethnic groups did not significantly differ in their mean difference in RT for Children as Sexual Beings, $F(2,97) 2.04, p=.136$, partial eta squared $=.040$, Dangerous World, $F(2,97) 2.99$, $p=.055$, partial eta squared $=.058$, Entitlement, $F(2,97) 0.76, p=.468$, partial eta squared $=.016$, nor Nature of Harm, $F(2,97) 0.58, \mathrm{p}=.564$, partial eta squared $=.012$. Interestingly, though, the ANOVA for Uncontrollable did demonstrate a significant interaction, $F(2,97) 4.13, p=.019$, partial eta squared $=.078$. Bonferroni post-hoc tests revealed that for Uncontrollable the mean difference in RT among Māori $(M=-115.75 \mathrm{~ms}$, 
$S D=199.62)$ was significantly different $(p=.015)$ to that among Others $(M=81.12 \mathrm{~ms}$, $S D=277.70)$. That is, Māori as a group appeared significantly less distorted than Others. Although Māori also appeared less distorted than Europeans $(M=-40.06 \mathrm{~ms}, S D=193.74)$ on Uncontrollable, the difference was not significant $(p=.364)$.

To summarise, the overall hypothesis was not supported by the data. CSOs did not demonstrate an offence-supportive pattern of responding compared to OCs and CCs when RTs to all ITs were combined. This pattern of results also held for RTs to Children as Sexual Beings, Dangerous World, Entitlement, and Nature of Harm. However, when RTs to Uncontrollable were compared, CSOs' RTs significantly differed from OCs'. That is, CSOs demonstrated offence-supportive responding for this IT, while OCs and CCs did not. Also, for Uncontrollable only, Māori participants showed a less distorted pattern of responding than participants in the "other" ethnic category.

\section{DISCUSSION}

It was predicted that CSOs would show a pattern of faster response times to ITconsistent target words than IT-inconsistent words relative to OCs and CCs. In the main, this hypothesis was not supported. Overall, CSOs did not respond faster to offencesupportive words than nonoffence-supportive words, and their response times to target words could not be differentiated statistically from the control groups'. For four out of the five IT categories (Children as Sexual Beings, Dangerous World, Entitlement, and Nature of Harm) this pattern of results held true. Interestingly, however, responses to the Uncontrollable IT revealed a pattern consistent with the proposed hypothesis. Here CSOs did respond faster to offence-supportive than nonoffence-supportive words, and their scores were significantly faster than OCs'.

Taken together, the findings of this study suggest that CSO participants did not hold offence-supportive beliefs. The null results emerged despite the fact that the method adopted in this study has successfully been used by other researchers to uncover evidence 
of different beliefs held within particular groups (e.g., Baldwin et al., 1993; Mikulincer, 1998). While these findings run contrary to the widespread hypothesis that CSOs hold offence-supportive beliefs, they do support a recent empirical study (Gannon et al., 2006) in which no evidence could be found that CSOs were misinterpreting offence descriptions in line with offence-supportive beliefs.

Questionnaire and interview studies frequently find evidence that CSOs endorse cognitive distortions. Rather than investigating cognitive distortions, in this and Gannon et al.'s study the aim was to observe the content of cognitive structures by studying participants' information processing. It appears that, overall, in neither study did CSOs process information in a manner suggesting that they held offence-supportive beliefs. Therefore the lack of evidence that CSOs hold offence-supportive beliefs found in this and Gannon's study suggest that CSOs' cognitive distortions may not be driven primarily by false beliefs. This in turn suggests that additional mechanisms might be at work; a finding consistent with recent proposals that social and self-protective phenomena (Maruna \& Mann, 2006), or judgment processes (Ward, Keown, \& Gannon, 2007) may play a role. Of course, the results of this and Gannon et al.'s study do not suggest that ITs or faulty core beliefs play no role in the generation of cognitive distortions. Rather, they indicate that faulty core beliefs underlie the cognitive distortions of only some CSOs.

The findings cast an interesting light on the IAT studies carried out by Mihailides et al. (2004), Gray et al. (2005) and Nunes et al. (2007). In the Mihailides et al. study, associations were found between CSOs' concept of sex and children, their concept of sex and uncontrollability, and, to a lesser degree, their concept of sex and entitlement. Likewise, Gray et al. and Nunes et al. found CSOs held associations between children and sex concepts. In each of these three studies the authors concluded that their results supported the idea that CSOs hold ITs that set them apart from others. In this study some evidence was found that the CSO sample held an Uncontrollable IT, but no evidence that 
a Children as Sexual Beings IT was held. This latter finding underscores the earlier point that holding associated concepts may be a different cognitive phenomenon to holding distorted beliefs.

Participants' responses to Uncontrollable were intriguing, as CSOs' mean scores significantly differed from OCs', but not CCs'. The Uncontrollable IT generated the most extreme scores from the offender groups, with CSOs producing their only mean positive (or offence-supportive) score, and OCs producing their most negative (or nonoffencesupportive) score. One could interpret the positive CSO response as supporting Ward's theory (Ward \& Keenan, 1999; Ward, 2000) that CSOs have beliefs that support propositions about one's behaviour being Uncontrollable. These beliefs may have been held by CSO participants prior to their offending and may have played an important causative role. However, if such beliefs do in fact have an aetiological role in child sexual offending this implies that the beliefs distinguish CSOs from general members of the population. Yet this is not what was found; CSOs' responses to Uncontrollable did not significantly differ from CCs'. This calls into question any interpretation of the current results as evidence that CSOs hold offence-supportive beliefs regarding uncontrollability.

The significant difference between CSOs' and OCs' responses might also be construed as evidence that CSOs hold an Uncontrollable IT. Yet this interpretation is again problematic, because the difference between the two offender groups is partly attributable to OCs having such a strong negative response to the Uncontrollable IT. Exactly why OCs' responses were so negative for this IT is unclear. Perhaps it relates to the fact that the OC sample was predominantly comprised of men convicted of violent acts ( $76 \%$ of OCs had committed at least one violent offence). At some stage in their history of incarceration a number of these men may have received anger management therapy that involved modification of beliefs pertaining to Uncontrollable. Unfortunately, the treatment experiences of offender controls were not recorded, so it is difficult to ascertain 
what effect, if any, exposure to treatment may have had on offender controls' Uncontrollable responses.

Another explanation exists for the difference between OCs' and CSOs' Uncontrollable responses. Coping with the demands of a prison environment (where self protection is important and sexual activity is tightly constrained) may have had different effects on the belief systems of OCs and CSOs. OC participants' belief in their ability to look after themselves and control their sexual urges might have been reinforced under such conditions. In contrast, the CSO sample might have had prison experiences that limited their opportunities to develop these beliefs (e.g., receiving constant threats from other prisoners may have led to a sense of vulnerability and reflecting on the nature of their crimes may have prevented formation of beliefs that sexual impulses can be controlled).

Yet another possibility is that the significant difference between OCs' and CSOs' Uncontrollable responses is linked to cultural differences. With Māori comprising 49\% of the OC sample, compared to $16 \%$ of the CSO and $14 \%$ of the CC samples, the difference in Uncontrollable scores might be attributable to Māori participants holding beliefs that oppose Uncontrollable. This suggestion is somewhat supported by the fact that for Uncontrollable Māori were less distorted (although not significantly so) than European New Zealanders and significantly less distorted than other non-European New Zealanders. Unfortunately, consideration of the cultural factors that might lead Māori to hold beliefs that oppose the Uncontrollable IT lies outside the remit of this thesis. However, consultation with Māori cultural advisors has uncovered the suggestion that for Māori men, among whom rates of offending are proportionately high (McFarlane-Nathan, 1999), offending may be associated with a sense of gaining control over adverse life conditions. Thus Māori prisoners may have a strong sense that they do guide their own destinies and have high self control. The possibility that Māori differ in some of the beliefs captured 
within Ward and Keenan's (1999) IT theory is an avenue worth exploring in future studies. Further experimental research is also needed to clarify whether the difference in cognitions regarding Uncontrollable between CSOs and other offenders is a widespread phenomenon.

A potential limitation in this study is that some participants may have deduced task aims and deliberately responded to some words slower than others. However, it seems unlikely that participants surmised that responding slower to offence-supportive words would make them look less distorted. In fact, verbal feedback from participants on completion of the task indicated general confusion about the aim of the experiment, with some participants expressing doubt that it could tell the experimenter anything useful at all. Furthermore, having a mere $2000 \mathrm{~ms}$ in which to respond would have made it very difficult for participants to decide whether or not they were viewing a real word and decide whether or not to deliberately stall each response. Nevertheless, some individuals did get removed from this study because they made too many errors or nonresponses, and a disproportionate number of these were CSOs. Perhaps the excluded participants made mistakes because they were distracted by concerns about what the experiment might reveal regarding their cognitions, and this could have biased overall group results. Unfortunately there is no simple way of confirming or denying this possibility.

This study could have been improved by asking all participants to complete a cognitive distortion questionnaire so that their responses could be compared with their LDT scores. Showing conclusively that CSO participants had higher levels of endorsement than control groups on cognitive distortion items would have strengthened the claim that cognitive distortion endorsements do not necessarily reflect faulty beliefs. On the other hand, as noted in the introduction to this thesis, interview studies almost invariably find that CSOs utter cognitive distortions, and questionnaires typically find that CSOs endorse more cognitive distortion items than controls, making it likely that had 
CSO participants been tested for the presence of cognitive distortions using conventional methods they would have appeared distorted. The fact that the sample was composed primarily of extrafamilial CSOs also makes it likely that the CSO sample would have endorsed cognitive distortions, since extrafamilial CSOs are commonly reported as being more cognitively distorted than intrafamilial CSOs (Fisher et al., 1999; Vanhouche \& Vertommen, 1999; Ward, 2000).

Regardless of any potential limitations, this study certainly adds weight to recent calls by researchers to fully assess the assumption that all cognitive distortions reflect underlying offence-supportive beliefs (see Gannon \& Polaschek, 2006; Maruna \& Mann, 2006; Ward, Keown, \& Gannon, 2007). Further research needs to be conducted that explores other drivers of cognitive distortions. For example, by manipulating the personal and interpersonal circumstances in which cognitive distortions are measured, psychologists may reach a greater understanding of the types of processes, both internal and external to CSOs, that determine whether they are likely to endorse or articulate a proposition that appears "distorted" to onlookers.

Another avenue for exploration is to investigate the effects of priming when using the LDT to study CSOs. Priming involves increasing the accessibility of a mental representation (e.g., a schema, belief or stored information structure) by exposing an individual to a relevant concept, word, or environmental cue (see Baldwin, Carrell \& Lopez, 1990; Bargh \& Chartrand, 1999; Higgins, 1989). In the current study it was assumed that CSOs' offence-supportive beliefs would be sufficiently accessible that upon reading context sentences their offence-supportive beliefs would activate and lead them to interpret sentences differently than controls. However, it is possible that prior, stronger priming was needed to activate CSOs' beliefs and thereby generate abnormal interpretations. For instance, CSOs may have responded differently if they had been 
primed by sexually or emotionally salient cues before completing the LDT. In Study Two this idea is explored.

Future research should also be directed at implementing other cognitive experimental techniques in the study of sexual offenders' implicit cognitions. Cognitive psychology has produced a wide range of experimental techniques designed to shed light on individuals' information processing styles and the contents of their beliefs and schemas. Thus far, very few of these techniques have been implemented in the study of sexual offenders. Therefore, the continued application of cognitive experiments in the study of CSOs is sorely needed.

In conclusion, the findings from this study have called into question the idea that CSOs articulate and/or endorse cognitive distortions because they hold abnormal, offencesupportive beliefs. This study has underscored the need for alternative research methods to be used in conjunction with questionnaire-based approaches when trying to understand the cognitive processes of CSOs. In addition, it has indicated that researchers and clinicians should continue to strive for a better understanding of the mechanisms underlying cognitive distortions. 


\section{Chapter Five}

\section{A Study of the Effects of Visual Priming on Information Processing in Child Sexual Offenders}

\section{Introduction}

Contextual and environmental variables can increase the likelihood that an individual will take cognitive short-cuts rather than engaging in effortful processing (Bargh, Lombardi, \& Higgins, 1988; Kunda, 1999). The use of even highly accessible schemas can be influenced by these external factors. In the case of sexual offenders, it has been proposed that a variety of important external stimuli serve to increase cognitive load, and decrease motivation and ability to engage in effortful, data-driven information processing. For instance, very positive or negative affective states, sexual arousal, and intoxication have all been said to increase the likelihood that sexual offenders will process information according to pre-existing schema shortcuts (Gannon \& Polaschek, 2006; Ward et al., 1997).

An interesting explanation for the null results reported in the previous chapter (and those found by Gannon et al., 2006) could be that CSOs' belief structures were not appropriately primed. When beliefs are primed they can affect subsequent information processing. For instance, Baldwin et al., (1990) found that visually priming either negative or positive beliefs about the self in a group of students altered the way that those students evaluated their own research ideas. More recently, Murray, Spadafore, and McIntosh (2005) demonstrated that activation of just-world beliefs affected participants' processing of rape-related information. Murray et al. primed half of a group of female participants by presenting them with rape-related words, before all participants read a dating scenario that was ambiguous in terms of the male dater's aggressiveness and the female dater's provocative behaviour. The authors found that participants holding strong just-world 
beliefs perceived the woman dater more negatively after being primed with rape-related words than those who were primed with neutral words.

In the study presented in Chapter Four it was hoped that as CSOs read context sentences (e.g., "A child who is fondled by an adult will probably be...") they would interpret those context sentences in line with offence-supportive beliefs and would therefore respond faster to the IT-consistent completion word "okay", for example, than to the IT-inconsistent completion word "hurt". While it was hoped that each context sentence would be a sufficient cue to activate an offence-supportive belief, it is possible that prior, stronger priming was needed in order for CSOs to make such faulty interpretations.

\section{Study Two: The Effects of Visual Priming on Interpretation of Ambiguous Sentences Related to Child Behaviour}

In the current study, attempts to activate beliefs regarding Children as Sexual Beings are made using visual primes. A memory-based study is then conducted to investigate whether CSOs hold the Children as Sexual Beings IT. Focussing specifically on Children as Sexual Beings (rather than all five ITs, as was done in Study One) allows in-depth examination of a single IT in each participant. Children as Sexual Beings has been selected as the most appropriate IT to investigate because it is thought to be CSO specific (Ward et al., 2007), it is the IT most widely represented in commonly-used cognitive distortion questionnaires (Gannon et al., in press), and Marziano et al.'s (2006) self-report study found that it was the IT most frequently endorsed by CSOs. Focussing on an IT that is thought to be CSO-specific has also obviated the need for CCs in this study, as CSOs' beliefs should appear different to all controls' for this IT (recall also that in Study One offender controls responded in a more, albeit nonsignificantly, IT-inconsistent manner than community controls).

The current study contains three successive stages: priming, encoding, and recognition. In the priming phase, CSOs' offence-supportive beliefs are primed by 
presenting CSOs and OCs with computerised images of semi-clothed children. Total time spent viewing such images is a significant predictor of sexual interest (Abel, Huffman, Warberg, \& Holland, 1998; Gress, 2005; Harris, Rice, Quinsey, \& Chaplin, 1996). That is, researchers have found that CSOs tend to view images of unclothed or semi-clothed children longer than non-CSO comparisons do. For instance, Gress (2005) measured the amount of time that 19 CSOs and seven adult sexual offenders spent viewing slides of nude and clothed males and females ranging in age from five years to adult. Gress reported that viewing times for both nude and clothed stimuli accurately predicted age and gender preference classifications when compared to participants' past sexual behaviour. Thus, if CSOs spend lengthy time periods looking at the images of children, presumably their belief structures should be activated, leading them to make sexualised interpretations of children's actions.

The subsequent two stages in this study are adapted from a previous design used successfully by Copello and Tata (1990) to investigate the manner in which violent offenders interpret ambiguous (but potentially violence-related) sentences. Copello and Tata asked participants to read a series of sentences (encoding phase) before surprising them with a memory task (recognition phase). For each ambiguous sentence shown during encoding, one of two disambiguated violent and non-violent versions were shown during recognition. As predicted, non-offender controls did not recognise violent versions of the ambiguous sentences as often as violent offenders did. More recently, Copello and Tata's design has been used to demonstrate cognitive biases towards aggressive stimuli among users of the drug MDMA (Curran, Rees, Hoare, Hoshi, \& Bond; 2004). While Curran et al. did not find that MDMA users recognised more violent sentences than non-users, MDMA users did show faster reaction times to violent sentences and were more confident that they had previously seen them. 
In the current study, sentences describing children's behaviour are used in the encoding and recognition tasks in place of Copello and Tata's violence-related sentences. During the encoding phase participants are asked to read ambiguous sentences about children that could be interpreted in a sexualised manner. Finally, participants are given a surprise recognition task in which they read slightly different versions of sentences presented during encoding; half of the originally sexually-ambiguous sentences are presented with a clear sexualised interpretation, while the other half are presented with a clear nonsexual interpretation.

The first hypothesis is that viewing time in the child priming condition will be longer for CSOs than OCs, while viewing time in the adult control condition will be statistically equal for both participant groups. The second hypothesis is that, compared to controls, CSOs in the priming condition will recognise more sexualised versions of the unambiguous sentences than benign versions. Because individuals are faster to process schema-congruent information (e.g., Fincher-Kiefer, 1992; Sharkey \& Sharkey, 1987), and in line with the findings of Curran et al. (2004), it is also hypothesised that this biased recall for sexualised sentences will be accompanied by faster responses among primed CSOs, compared to OCs and unprimed CSOs.

\section{METHOD}

\section{Participants}

Sixty-four men took part in this study: 27 CSOs and 37 OCs. Participants were recruited from New Zealand Department of Corrections prisons. Prison managers distributed a recruitment flyer to prisoners inviting them to take part in "a reading study" and "an internet study". As with Study One, flyers stated that participants were wanted who could read the recruitment flyer with ease. Also, the volunteer rates in this study again could not be monitored. Twenty-two CSOs and 29 OCs who took part in this study 
also participated in Study One. Selection criteria and demographic details for each participant group follows.

\section{Group selection criteria and demographic details}

CSOS

CSOs were prison inmates whose index offences included at least one sexual conviction against a person under the age of 16 . Their ages ranged from 22 to 77 years $(M=45.85$, $S D=14.61)$ and their victims' ages ranged from 2 to 15 years $(M=9.52, S D=4.33)$. According to CSOs' conviction records, 16 were extrafamilial offenders (i.e., had molested at least one non-relative), while 11 were intrafamilial offenders (i.e., had molested only biological or non-biological family members). Two of the extrafamilial and none of the intrafamilial CSOs had received cognitive-behavioural therapy to reconstruct distorted offence-related cognitions. Since receiving therapy, the two treated CSOs had committed at least one sexual assault against a child. The mean Static-99 score for all CSO participants indicated a medium-low recidivism risk level $(M=2.85, S D=2.25)$ according to criteria set by Phenix et al. (2000). The number of years that CSOs had spent in formal education ranged from nine to 15 years $(M=10.26, S D=1.56)$. Five CSOs identified themselves as Māori, 15 as European, and seven as "other".

OCs

OCs were men who were serving a prison sentence, but who had never been convicted of a sexual offence against a person under the age of 16. OCs were aged from 20 to 69 years $(M=34.16, S D=9.63)$. Conviction records showed that six OCs had been convicted at some time for a sexual offence against a person over the age of 16 . The number of years that $\mathrm{OC}$ participants had spent in formal education ranged from nine to 12 years $(M=10.00, S D=1.05)$. Seventeen OCs identified themselves as Māori, 16 as European, and five as "other". 
Independent sample t-tests revealed the two offender groups did not differ significantly on number of years spent in education or length of prison sentence. A Chisquare test for independence confirmed that between-group differences approached significance for ethnicity, $\chi^{2}(2, N=64)=5.98, p=.05$, Cramer's $\mathrm{V}=.306$. CSOs and OCs differed significantly on age, $t(62)=3.86, p<.001$, with CSOs having a higher mean age $(M=45.85, S D=14.61)$ than OCs $(M=34.16, S D=9.63)$. Analyses of Variance (ANOVAs) were run to see whether differences existed across the four participant groups (i.e., primed and control CSOs and OCs). The four groups did not differ significantly on number of years spent in education and length of prison sentence, and a Chi-square test for independence confirmed that between-group differences did not exist for ethnicity. Also, an independent samples t-test confirmed that CSOs did not differ in their Static-99 scores across the two conditions. However, the groups were found to significantly differ in age, $F(3,60)=7.23, p<.001$. Bonferroni post-hoc tests showed that primed CSOs were significantly older $(M=51.91, S D=11.53)$ than control CSOs $(M=41.00, S D=15.34)$, primed OCs $(M=34.30, S D=8.39)$, and control OCs $(M=34.00, S D=11.19)$. Characteristics of the four participant groups are displayed in Table 5.1. 
Table 5.1

Demographic details for participant groups: Study Two

\begin{tabular}{|c|c|c|c|c|}
\hline & \multicolumn{2}{|c|}{ Child Sex Offenders } & \multicolumn{2}{|c|}{ Offender Controls } \\
\hline & $\begin{array}{c}\text { Child Prime } \\
n=12\end{array}$ & $\begin{array}{c}\text { Adult Prime } \\
n=15\end{array}$ & $\begin{array}{c}\text { Child Prime } \\
n=20\end{array}$ & $\begin{array}{c}\text { Adult Prime } \\
n=17\end{array}$ \\
\hline \multicolumn{5}{|l|}{ Age (years) } \\
\hline Mean & 51.92 & 41.00 & 34.30 & $34.00 * * *$ \\
\hline$S D$ & 11.53 & 15.34 & 8.39 & 11.19 \\
\hline \multicolumn{5}{|c|}{ Years in education } \\
\hline Mean & 10.08 & 10.40 & 9.90 & 10.12 \\
\hline$S D$ & 1.83 & 1.35 & 0.97 & 1.17 \\
\hline \multicolumn{5}{|l|}{ Ethnicity (n) } \\
\hline Maori & 3 & 2 & 9 & 8 \\
\hline European & 7 & 8 & 10 & 6 \\
\hline Other & 4 & 3 & 1 & 3 \\
\hline \multicolumn{5}{|c|}{ Reading time (ms) } \\
\hline Mean & 3476.77 & 4055.11 & 3092.29 & 3553.09 \\
\hline$S D$ & 2349.74 & 3584.54 & 1332.25 & 3115.36 \\
\hline \multicolumn{5}{|c|}{ Violent Convictions (n) } \\
\hline Mean & 1.67 & 1.13 & 3.45 & 3.71 \\
\hline$S D$ & 1.67 & 1.96 & 4.01 & 4.10 \\
\hline \multicolumn{5}{|l|}{ CSO type (n) } \\
\hline Intrafamiliail & 5.0 & 6.0 & - & - \\
\hline Extrafamilial & 10.0 & 6.0 & - & - \\
\hline \multicolumn{5}{|c|}{ Child vicitms (n) } \\
\hline Mean & 3.00 & 3.07 & - & - \\
\hline$S D$ & 2.95 & 2.71 & - & - \\
\hline \multicolumn{5}{|c|}{ Victim age (years) } \\
\hline Mean & 10.13 & 11.47 & - & - \\
\hline$S D$ & 3.97 & 3.37 & - & - \\
\hline \multicolumn{5}{|c|}{ STATIC-99 Score } \\
\hline Mean & 2.67 & 3.00 & - & - \\
\hline$S D$ & 2.02 & 2.48 & - & - \\
\hline
\end{tabular}




\section{Ethical Considerations}

New Zealand Department of Corrections and Victoria University of Wellington approved all procedures and materials used in this study. Before testing began all participants read (or were read) an information sheet outlining the study procedure and their rights as participants (which met NZPS Code of Ethics criteria). Participants were told they would need to provide details of their offending, which an experimenter would verify by checking their prison records on completion of testing. Participants signed a consent form before taking part in the experiment and were sent a combined debriefing and results summary sheet once results were collated.

\section{Design}

A Mixed Subjects Experimental Design was used. The overall study design is depicted in Figure 5.1

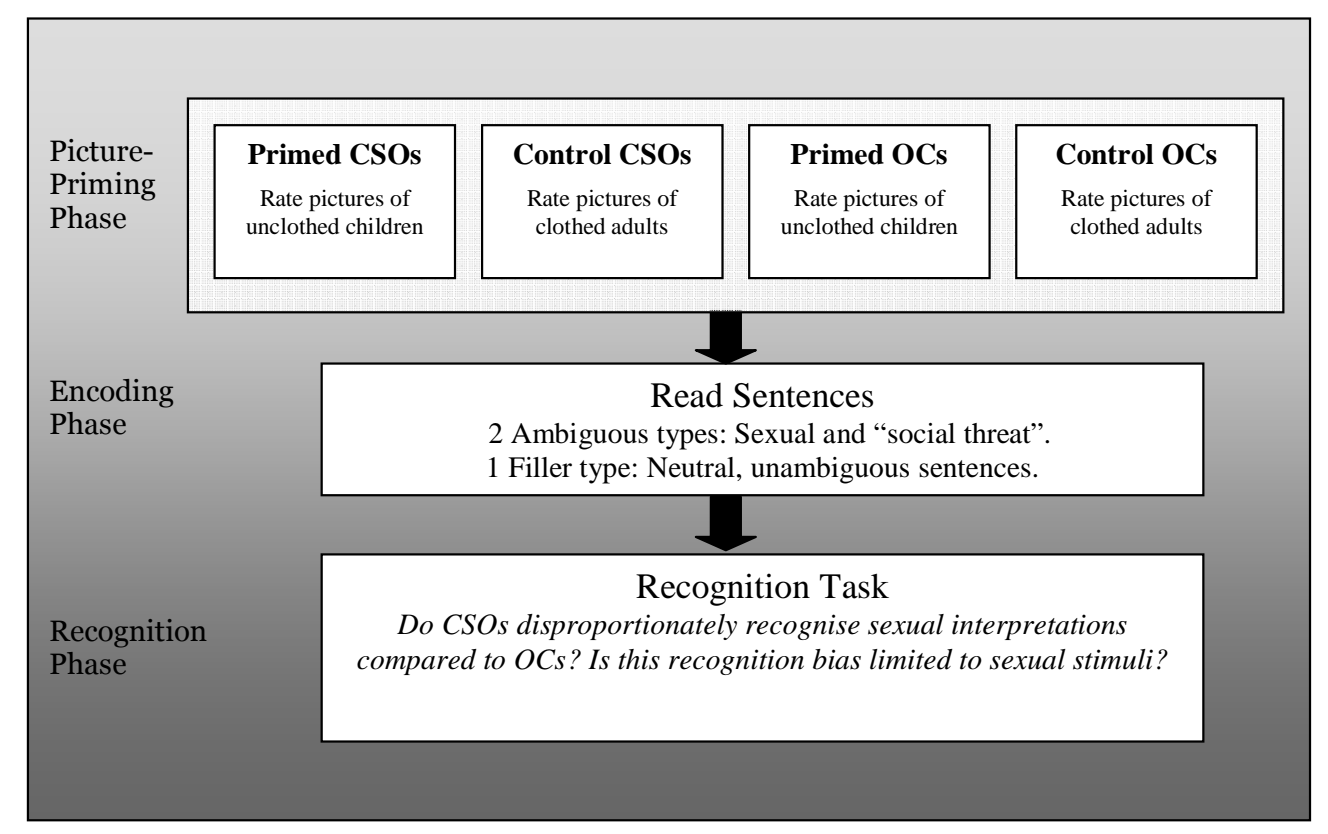

Figure 5.1. Study Two Experimental Design 
There were three experimental phases: priming, encoding, and recognition.

\section{Priming}

Half the CSOs and OCs viewed pictures of semi-clothed children twice. The other half viewed pictures of clothed adults twice. Pictures were presented twice to ensure adequate exposure. To disguise task aims, this phase was described to participants as an 'Age Judging Task' in which the task aim was to indicate whether depicted subjects were over or under a particular age (see Priming Phase in upcoming 'Procedure' section).

\section{Encoding}

Participants viewed 20 ambiguous and ten filler sentences.

\section{Recognition}

Participants viewed 50 sentences similar to those viewed during encoding and indicated whether they recognised each sentence.

A mixed subjects design was used. Independent between-subjects variables were group (CSOs vs. OCs) and condition (priming vs. control). Dependent variables were picture response time (during the age-judging task), comparative recognition scores for recognition sentences, and reaction time to recognition sentences.

\section{Materials}

\section{Priming pictures}

Child priming pictures were selected from computer-generated images (available commercially from Pacific Psychological Assessment Corporation, 2004). They were constructed via computer morphing technology designed for use in studies of sexual arousal to children. Females were depicted wearing swimming costumes; males wore boxer shorts. Pictures were selected that the researchers deemed to be maximally attractive and provocative in terms of stance and facial expression. Twenty images were presented, comprised of five images from each of four categories: females and males aged approximately nine and 13 years old (see Appendix Three for example images). Adult 
control pictures were photographs chosen from the International Affective Picture System (Center for the Study of Emotion and Attention, 1999). Ten females and ten males judged to be middle-aged or older were depicted fully-clothed (see Appendix Four for example photographs). These pictures were deemed being highly unlikely to arouse sexual interest and thus should not have primed cognitive structures relating to children or sex.

\section{Encoding sentences}

Twenty ambiguous and ten neutral "filler" sentences were used (see Appendix Five). Ten ambiguous sentences could be interpreted in a sexual or nonsexual way; these were target stimuli (e.g., "As 7-year old Jenny played cards, she showed her knickers”). These "sexual" sentences were designed to tap beliefs that children are sexual beings. In addition, ten sentences were presented that could be interpreted in a more generally threatening or nonthreatening way (e.g., "The doctor frowned as he measured little Sarah's growth"). These potentially threatening sentences (hereafter called "threat sentences") were based on sentences used by Copello and Tata (1990) and were presented to monitor whether group differences in responding reflected general negative biases. The ten remaining filler sentences were not ambiguous (e.g., "The two old friends played pool until closing time"). They were added to the task to conceal experiment aims and to monitor participant responding.

\section{Recognition sentences}

Fifty recognition sentences were used that were similar in wording and content to encoding sentences (see Appendix Six). Ten filler recognition sentences had identical meanings to the ten filler sentences presented at encoding but contained minor word changes (e.g., "The two friends played pool until the pub closed"). Of the forty unambiguous recognition sentences, ten presented a nonsexual interpretation of encoding sentences (e.g., "While Jenny played cards, she accidentally showed her knickers"), ten presented a sexual interpretation (e.g., "While Jenny played cards, she deliberately 
showed her knickers"), ten presented a nonthreatening interpretation (e.g., "The doctor frowned as he measured little Sarah's height"), and ten presented a threatening interpretation (e.g., "The doctor frowned as he measured little Sarah's cancer").

All sentences presented in the recognition and encoding phases were similar in length and complexity. An Analysis of Variance found that average Flesch Kincaid scores for each sentence type (sexual, threat, and neutral) were statistically equivalent in both the encoding phase, $F(2,27)=0.90, p=.419$, and the recognition phase, $F(2,57)=0.77, p=$ .466. Using t-tests it was found that average Flesch Kincaid scores in the recognition phase were equivalent between the disambiguated sexual and nonsexual sentences, $t(18)=$ $.46, p=.652$, and between the disambiguated threatening and nonthreatening sentences, $t(18)=.06, p=.952$.

\section{Apparatus}

All tasks were programmed using a Superlab package. The program was run on 12-inch colour monitor screen from a Dell Pentium Latitude D600 laptop computer. Written instructions and stimuli were centred on a white screen in bold black Times New Roman text (font size 40). Participants made their responses on a Cedrus Model RB-730 response pad. In the age judging task, labels were placed over keys on the response pad reading "under 16" and "over 16" in the priming condition and "under 75" and "over 75" in the control condition. These were replaced with labels reading "recall" and "don't recall" during the recognition phase. Placement of labels was carefully alternated across tasks and across participants to eliminate handedness effects.

Superlab controlled the random presentation of pictures in the age-judging task, and recorded the time that elapsed between each button press made to indicate an age judgment. Superlab also controlled the random presentation of encoding and recognition sentences, and recorded type of response made (recall/don't recall) and RT to recognition sentences. 


\section{Procedure}

\section{Priming phase}

Participants were tested individually, in a single session. Each testing session took approximately 45 minutes per participant. After giving written consent, participants supplied demographic information about themselves (e.g., age and ethnicity) and provided details about the nature and number of their crimes. Participants randomly allocated into the priming condition were told that on first presentation of the child pictures they should simply familiarize themselves with the pictures. On second presentation, however, they should use the respond pad to indicate whether the person in the picture was over or under the age of 16. They were advised to spend as long as they wanted looking at each picture. To disguise task aims, priming participants were told that the study was looking at trends in the use of internet pornography and that a research aim was to establish whether adult men can accurately deduce age in young people. Participants generally appeared to accept this as a task aim, with many commenting on the difficulty of currently distinguishing age in young people. Control participants were told that they were to view two sets of images of mature adults. As with priming participants, they were told to familiarize themselves with the pictures on first presentation and to indicate whether the depicted subjects were over or under 75 years of age on second presentation (in both conditions people in the images looked younger than the designated age threshold). Control participants were told the study aimed to investigate modern-day age perceptions.

Before the age-judging task all participants completed a practice run. To create a sense of privacy a large screen was placed around the computer, blocking it from the experimenter's view. This was ostensibly put up to ensure that good lighting conditions were available to all participants. To increase privacy the experimenter also faced away from the participant and perused some papers. 


\section{Encoding phase}

Participants were asked to take part in "a reading study". They were instructed to read sentences—taking as long as needed—that they would later be asked questions about. Participants read 30 sentences presented serially and in random order. Upon reading each sentence participants pressed a button on the response pad to view the next sentence.

\section{Recognition phase}

Participants next completed a surprise "memory test". They were presented with 30 recognition sentences: ten unambiguous versions of sexual sentences (i.e., five sexual and five nonsexual), ten reformulations of filler sentences, and ten unambiguous versions of threat sentences (i.e., five threatening and five nonthreatening). As each sentence appeared participants indicated whether they had seen that sentence (or one with the same meaning) during the encoding phase. Participants were told that all test sentences would be slightly different to the earlier sentences, so the aim was to indicate sentences that had the same meaning as prior sentences.

\section{RESULTS}

Analyses were conducted to explore the hypotheses that: (1) during the priming phase, primed CSOs will display longer viewing times for children than OCs or control CSOs; (2) during the recognition phase, compared to OCs and control CSOs, primed CSOs will remember more sexual than nonsexual recognition sentences; and (3) this biased recognition of sexual recognition sentences by primed CSOs will be accompanied by faster responses relative to other groups.

\section{Data Preparation: Reaction Times}

Two sets of RT data were of interest in the current analysis: (1) Time taken to indicate age judgments for pictures during the priming phase; and (2) Time taken to indicate sentence recollection during the recognition phase. 
As noted in Study One, RT data needs to be transformed such that the effects of outliers on ANOVAs are minimised (Ratcliff, 1993). Because RT data in the current study contained extreme responses that probably did not represent the intended cognitive mechanisms, and in line with previous research (e.g., Holden, Kroner, Fekken, \& Popham, 1992; Ratcliffe, 1993; Welford, 1980) both sets of RT data (i.e., (1) and (2)) were Windsorised to adjust for outliers.

For RT data set (2), mean RTs to unambiguous nonsexual sentences were subtracted from mean RTs to unambiguous sexual sentences to create a mean sexual RT score for each participant. Likewise, mean RTs to unambiguous nonthreatening sentences were subtracted from mean RTs to unambiguous threatening sentences to create a mean threat $R T$ score for each participant. This procedure clarified each participant's overall pattern of responding to sexual and threat sentences. A positive mean sexual RT score would indicate that a participant was faster to recognise sexual sentences relative to nonsexual sentences, while a positive mean threat RT score would indicate that a participant was faster to recognise threatening sentences relative to nonthreatening sentences.

\section{Data Preparation: Recognition Analysis}

Sentences presented in the recognition phase were grouped according to the following five categories: filler, nonthreatening, threatening, nonsexual, and sexual. Recognition scores for each category were calculated so that possible scores ranged from zero (no sentences recognised) to five (all sentences recognised). Next, mean recognition scores for unambiguous nonsexual sentences were subtracted from mean recognition scores for unambiguous sexual sentences to create a mean sexual recognition score for each participant. Likewise, mean recognition scores for unambiguous nonthreatening sentences were subtracted from mean recognition scores for unambiguous threatening sentences to create a mean threat recognition score for each participant. This procedure 
clarified each participant's overall pattern of responding to threat and sexual sentences. A positive mean sexual recognition score would indicate that a participant had recognised more sexual than nonsexual sentences, while a positive mean threat recognition score would indicate that a participant had recognised more threatening than nonthreatening sentences.

\section{Main analysis of picture viewing times}

To see whether viewing time during the age judging task differed between the two offender groups (CSO vs. OC) according to the type of picture presented (priming vs. control) two independent samples t-tests were conducted. The first t-test compared the length of time that CSOs and OCs looked at control pictures. As expected, no significant difference in looking times was found between the two groups $t(30)=.08, p=.934$. The second t-test compared the length of time that CSOs and OCs looked at priming pictures. Here a significant difference was found between CSOs' and OCs' looking times, $t(30)=$ 2.44, $p=.021$, partial eta squared $=.166$, with CSOs viewing the child pictures considerably longer $(M=1787.08 \mathrm{~ms}, S D=871.81 \mathrm{~ms})$ than OCs $(M=1200.97 \mathrm{~ms}, S D=$ 492.21ms). Thus, CSOs displayed longer viewing times than OCs in the priming condition only.

\section{Main analysis of sentence recognition}

The crucial question of whether primed CSOs showed a recognition bias for unambiguous sexual sentences was first investigated. A two-way ANOVA was conducted to see whether experimental condition (priming vs. control) differentially affected the offender groups (CSO vs. OC) in terms of the types of unambiguously sexual/nonsexual sentences they recognised. The independent variables were Offender Group (CSO and OC) and Condition (priming and control). The dependent variable was Mean Sexual Recognition Score. There was no main effect of Group, $F(1,60)=0.75, p=.391$, partial eta squared $=.012$, nor Condition, $F(1,60)=1.01, p=.947$, partial eta squared $<.001$. 
Also, contrary to predictions, no significant interaction between Offender Group and Condition emerged, $F(1,60)=2.53, p=.117$, partial eta squared $=.041$, illustrating that there did not appear to be a difference in participants' recognition of unambiguously sexual and nonsexual sentences (see Figure 5.2). In other words, primed CSOs $(M=-0.67$, $S D=1.07)$ did not have more positive Mean Sexual Recognition Scores than control CSOs $(M=-0.07, S D=1.83)$, primed OCs $(M=0.30, S D=1.49)$, and control OCs $(M=-$ $0.35, S D=1.62$ ). Thus, contrary to expectation, neither primed nor control CSOs showed a bias for sexual sentences.

An analysis was conducted to ensure that inclusion of intrafamilial CSOs did not account for the above nonsignificant interaction. An independent-samples t-test was used to compare extrafamilials' and intrafamilials' Mean Sexual Recognition Scores. It was found that no significant difference, $t(25)=-0.33, p=.742$, existed between extrafamilials' recognition scores $(M=-0.25, S D=1.44)$ and intrafamilials' recognition scores $(M=-0.45, S D=1.75)$, indicating that inclusion of intrafamilial CSOs did not account for the above null results. 


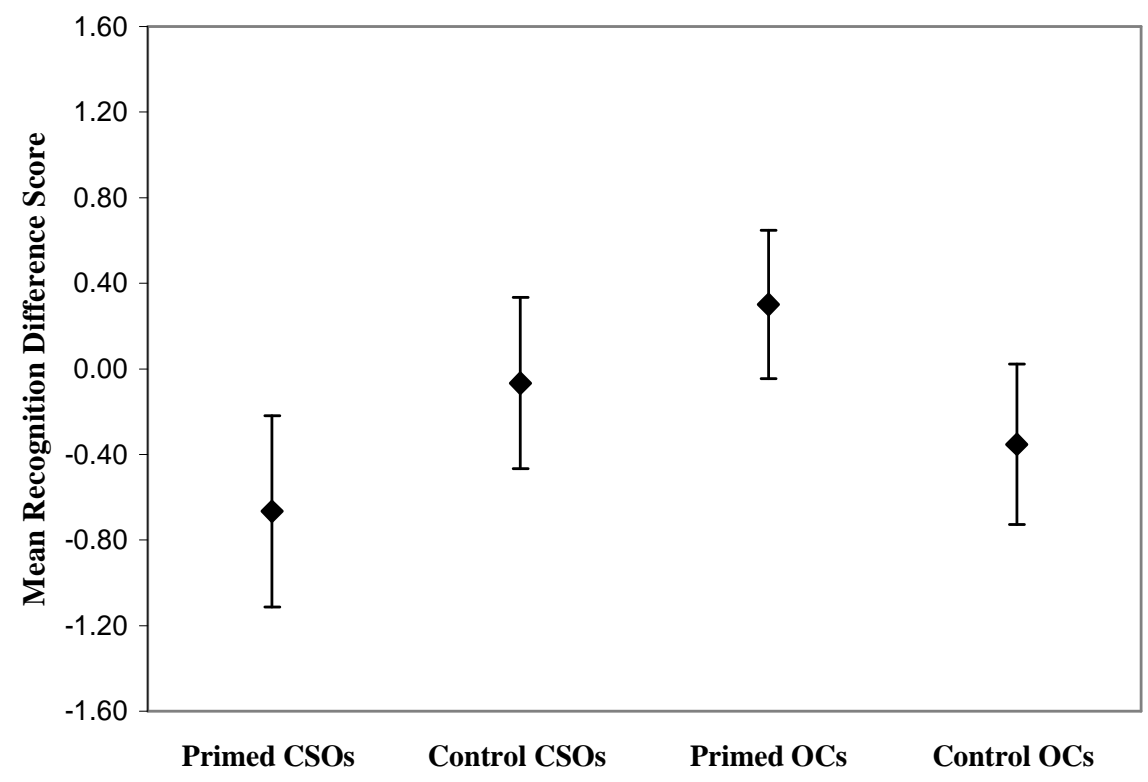

Figure 5.2. Mean Recognition Differences (+/- SEM) for Sexual Recognition Sentences.

Next participants' recognition scores for filler sentences were compared to ensure general recognition levels did not differ. A two-way ANOVA was conducted to see whether experimental condition (priming vs. control) differentially affected the offender groups (CSO vs. OC) in terms of the number of filler sentences they recognised. The independent variables were Offender Group (CSO and OC) and Condition (priming and control). The dependent variable was Mean Filler Recognition Score. There was no main effect of Group, $F(1,60)=1.55, p=.218$, nor Condition, $F(1,60)=2.36, p=.130$. As expected, no significant interaction between Offender Group and Condition emerged, illustrating that there did not appear to be a difference in the number of times that primed and control CSOs and OCs said they recognised filler sentences, $F(1,60)=2.99, p=.586$, partial eta squared $=.005$. Participants' Mean Threat Recognition Scores were also analyzed to ensure that general recognition biases for threatening sentences did not exist. 
The above two-way ANOVA was repeated with Mean Threat Recognition Score as the dependent variable. There was no main effect of Group, $F(1,60)=0.16, p=.695$, nor Condition, $F(1,60)=0.08, p=.773$. No significant interaction between Offender Group and Condition emerged, illustrating that there were no differences in the Mean Threat Recognition Scores of primed CSOs $(M=-0.08, S D=2.23)$, control CSOs $(M=0.60, S D$ $=1.64)$, primed OCs $(M=0.55, S D=1.85)$, and control OCs $(M=-0.41, S D=1.87)$, $F(1,60)=2.94, p=.092$, partial eta squared $=.047$. In other words, as expected, participant groups did not differ in their relative recognition of nonthreatening and threatening sentences.

Because CSOs were significantly older than OCs, all two-way ANOVAs described above were rerun with age as a covariate. The main pattern of results remained the same throughout (i.e. no significant interactions emerged). Also, to check that the above findings were unaffected by the fact that six OC participants had sexually offended against adults, the above two-way ANOVAs were rerun with data gathered from these six OCs removed from the analysis. Again, the pattern of results was unchanged.

\section{Main analysis of sentence reaction times}

If primed CSOs had recognised more sexual than nonsexual sentences compared to other participant groups, this would indicate that they had a processing bias towards sexual sentences and so should produce faster response speeds to sexual sentences. However, as the above analyses did not reveal a processing bias in primed CSOs towards sexual sentences, primed CSOs should not be faster to make a decision about sexual sentences relative to nonsexual sentences compared to OCs and control CSOs. In the following section this claim is investigated.

In order to investigate RTs to sexual sentences, the relative rates of responding participants had to unambiguous sexual and nonsexual sentences were of key interest. 
Therefore, a two-way ANOVA was conducted to examine whether experimental condition (priming vs. control) differentially affected offender groups (CSO vs. OC) in their Mean Sexual RT Scores. Main effects were not found for Offender Group, $F(1,60)=0.67, p=$ .417 , nor Condition, $F(1,60)=1.21, p=.275$. In addition, an Offender Group x Condition interaction was not found, $F(1,60)=0.49, p=.488$, partial eta squared $=.008$. Thus it was found that primed CSOs $(M=6987.05 \mathrm{~ms}, S D=3418.96 \mathrm{~ms})$ were no quicker than primed OCs $(M=5889.74 \mathrm{~ms}, S D=3348.10 \mathrm{~ms})$, unprimed CSOs $(M=5683.59 \mathrm{~ms}, S D$ $=2360.42 \mathrm{~ms})$, and unprimed OCs $(M=5597.09 \mathrm{~ms}, S D=2041.20 \mathrm{~ms})$ to react to unambiguous sexual sentences relative to nonsexual sentences (see Figure 5.3).

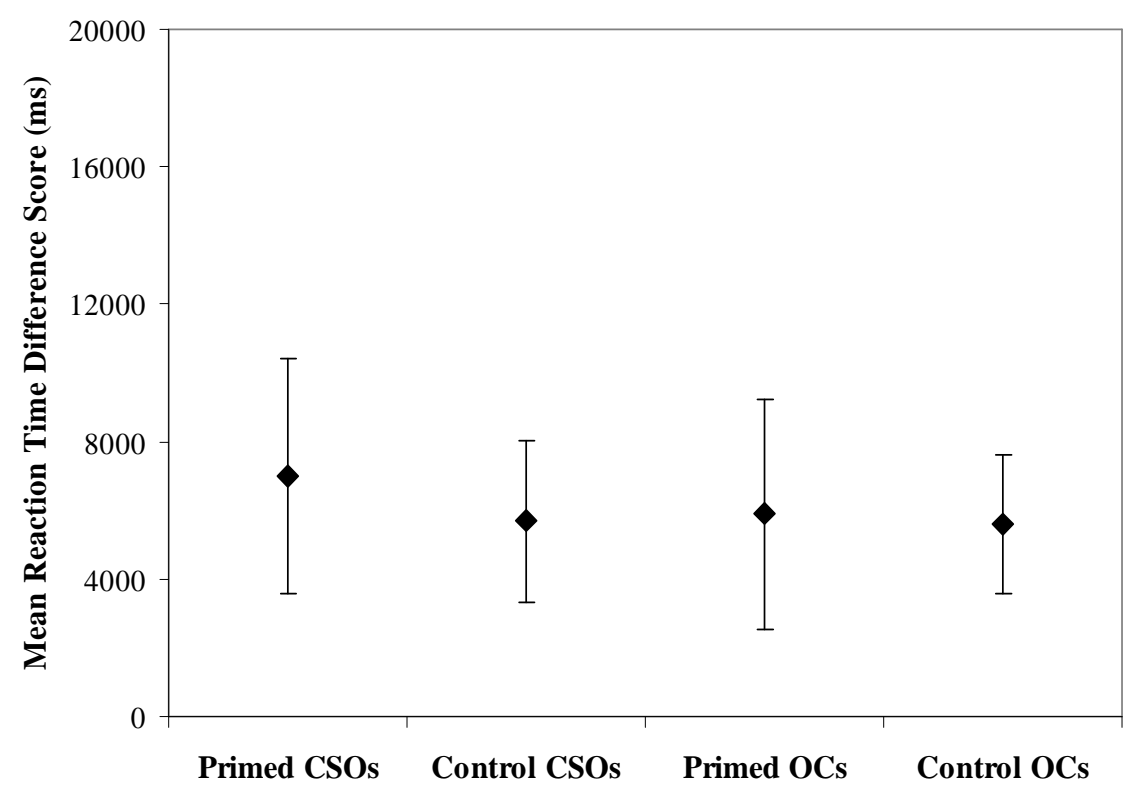

Figure 5.3. Mean Reaction Time Differences (+/- SEM) in Milliseconds (ms) for Sexual Recognition Sentences.

To first ensure that CSOs and OCs in the priming and control conditions did not differ in their baseline sentence processing speed, a two-way ANOVA was conducted to 
examine whether experimental condition (priming vs. control) differentially affected offender groups (CSO vs. OC) in their RTs (in milliseconds) to filler sentences. The independent variables were Offender Group (CSO and OC) and Condition (priming and control). The dependent variable was Mean Filler RT Scores. There was no main effect of Group, $F(1,60)=1.48, p=.229$, nor Condition, $F(1,60)=0.22, p=.639$. No significant differences existed in RTs to filler sentences between primed and control CSOs and OCs across the two conditions, $F(1,60)=.824, p=.368$, partial eta squared $=.014$. Participants' Mean Threat RT Scores were also analyzed to ensure that general RT biases for threatening sentences did not exist. The above two-way ANOVA was repeated with Mean Threat RT Score as the dependent variable. There was no main effect of Group, $F(1,60)=2.52, p=.118$. However, there was a main effect for Condition, $F(1,60)=8.31$, $p=.005$, with participants in the priming condition showing a more negative Mean Threat RT Score $(M=-902.17 \mathrm{~ms}, S D=1744.84 \mathrm{~ms})$ than participants in the control condition ( $M$ $=571.58 \mathrm{~ms}, S D=2426.08 \mathrm{~ms}$ ). As expected, no significant differences existed in the relative times CSOs and OCs in the priming and control conditions took to respond to threatening and nonthreatening sentences, $F(1,60)=.02, p=.897$, partial eta squared < .001 .

Because CSOs were significantly older than OCs, all two-way ANOVAs involving RTs were rerun with age as a covariate. Once again, no significant interactions emerged.

\section{DISCUSSION}

In line with the first hypothesis, CSOs and OCs viewed images of clothed adults for equivalent lengths of time, while CSOs viewed images of semi-clothed children longer than OCs did. This finding accords with studies in which CSOs have shown longer viewing latencies than comparison groups when presented with clothed or unclothed images of children (e.g., Abel et al., 2004; Harris et al., 1996). As research has demonstrated, viewing time is a reliable measure of sexual interest (e.g., Abel, et al., 1998; 
Abel et al., 2004; Gress, 2005; Harris et al., 1996). Thus, the protracted priming picture viewing time found for CSOs in this study appears to indicate CSOs experienced sexual interest when exposed to images of children. This suggests that the priming stimuli simulated cues encountered by many CSOs during the offence process, which presumably activated CSOs' beliefs linking children and sexual behaviour.

Our second hypothesis was that CSOs in the priming condition would demonstrate preferential recognition for unambiguously sexual sentences compared to controls. Interestingly, this result did not emerge. Primed and control CSOs and OCs showed no difference in recognition levels for any sentence type nor reaction times to any sentence type. While it comes as no surprise that CSOs didn't differ from controls in the way they remembered filler and threat sentences, it was expected that primed CSOs would interpret ambiguously sexual sentences in line with beliefs that children are sexual beings, and hence would recognise a greater number of sexualised versions of these sentences than controls, or at least respond faster to sexualised sentences. The null result in this regard does not accord with the idea formalised by Ward (Ward, 2000; Ward \& Keenan, 1999) that CSOs tend to hold beliefs that children are sexual agents who invite and enjoy sexual activity. In short, the CSOs in this study showed no evidence of holding these types of beliefs, even when exposed to a prime that should have activated beliefs linking children and sex. Previous studies that have used words or images to prime participants' beliefs have demonstrated subsequent changes in the way that participants process and respond to related stimuli (Baldwin et al., 1990; Murray et al., 2005). Even in studies that haven't utilised priming, the current experimental design has found expected group differences in responses to disambiguated sentences, both in terms of the groups' recognition rates (Copello \& Tata, 1990), and reaction times (Curran et al., 2004). The fact that comparable results did not emerge in the current study raises questions about the extent to which the CSO participants held stable beliefs that children are sexual beings. 
Similar doubts were raised by the previous study reported in this thesis, in which evidence of beliefs linking sex and children could not be detected in CSOs' responses to a lexical decision task. The current results also align with those of Gannon et al. (2006), who embedded ambiguous sentences in a vignette describing events surrounding the sexual abuse of a child. The sentences could be interpreted in line with four ITs, including the Children as Sexual Beings IT. Contrary to Gannon et al.'s expectations, CSOs did not recall these sentences in a markedly different way than controls did, leading Gannon et al. to surmise that the CSOs did not hold beliefs supporting Children as Sexual Beings.

While Study One used stimuli that tapped all five of Ward and Keenan's (1999) ITs, the sentences used in the current study only tapped beliefs contained within the Children as Sexual Beings IT. Of the five ITs identified by Ward and Keenan, the Children as Sexual Beings IT was found to be the most prevalent among CSOs in Marziano et al.'s (2006) self-report study, with all but one CSO endorsing this IT in an interview study (Marziano, 2002). Also, Children as Sexual Beings is the belief cluster most widely represented in commonly-used cognitive distortion questionnaires (Gannon et al., in press). Having said that, it would seem unreasonable to expect all CSOs in the current sample to hold beliefs that children are sexual beings, and one could argue that this made it difficult to detect a statistical difference between primed CSOs and other participant groups in this study. However, a perusal of the mean recognition scores for nonsexual and sexual sentences for each participant group reveals no bias towards recall of sexual sentences between primed CSOs and the other three participant groups. Because the Children as Sexual Beings IT is hypothesised to be offence-specific, one would surmise that in the current study primed CSOs should have demonstrated at least a small bias towards sexual sentences relative to OCs.

Nevertheless, the above issue raises an important point: Ward and Keenan (1999) have stated that individual CSOs hold varying numbers of ITs. That is, each CSO does not 
necessarily hold all five ITs. Yet in Study One and the current study CSOs as a group have been compared with controls to see if they respond differently to material tapping ITs. One would expect that for any IT there will be a subset of CSOs who, according to conventional measures of offence-supportive beliefs, do not appear to hold beliefs aligning with that particular IT. Perhaps when group differences are studied between CSOs and controls for any particular IT, the subset of CSOs who don't support that IT on conventional measures are obscuring the distorted responding of other members of the CSO group. Research is needed to see whether CSOs can be distinguished from OCs in cognitive experiments when this important issue is addressed. Study Three in this thesis takes this issue into account.

It is also possible that CSOs in the current study behaved defensively by deliberately pretending not to recall unambiguously sexual recognition sentences. However, other research suggests that when individuals "fake good" on similar tasks their reactions are typically faster than when they do not fake good (see Gannon \& Polaschek, 2005). Participant groups in the current study could not be distinguished according to their reaction times to any particular class of sentence, seeming to indicate that no group was deliberately manipulating responses.

Another limitation concerns the small CSO participant sample obtained in this study. Although the interactions concerning sexual sentences did not even approach significance, ideally a larger number of CSOs would have been included in this study in order to increase statistical power. Interestingly, the results depicted in Figure 1 indicate that not only were differences between experimental groups nonsignificant, but the results did not follow an expected pattern, as primed OCs said they recognised a greater ratio of sexual sentences than primed CSOs (even though OCs had shown no signs of sexual interest in the child pictures). Although the fact that this difference was nonsignificant indicates that it is best explained by chance, it is possible that viewing the child pictures 
somehow affected offender controls' perceptions of sentences describing children's actions (e.g., the pictures may have triggered beliefs that children can behave manipulatively or place one in danger).

In summary, it is possible that the null results found in this study are attributable to experimental design flaws, or to the fact that the experimental manipulation was not sensitive enough to detect differences between groups. However, it appears more likely that CSOs in the current study did not hold beliefs that paint children as sexual beings.

Future research should continue to apply cognitive-experimental techniques to the study of CSO cognition. Currently the implementation of these implicit techniques within the field of sexual offending is in its infancy. It is vital that psychologists understand whether the current lack of experimental evidence for CSO-specific beliefs reflects the fact that such beliefs are less widespread than currently thought, or whether it reflects the need for researchers to refine their techniques and develop more sophisticated ways of applying them. For instance, studies are needed that explore in greater depth the effects of alternative primes (e.g., emotion, mood, and sexual arousal manipulations) on information processing in CSOs.

There is also a need to develop experimental stimuli that more accurately imitate situations likely to be experienced by CSOs in their day-to-day lives. It is possible that in the current study the experimental task given to participants was too far removed from a real-life situation to reveal information-processing biases. Clearly, reading brief sentences about children is not equivalent to interpreting the complex social data available in reallife interactions with children. Of course, ethical considerations rule out the possibility of any direct contact between children and untreated CSOs, but in future more realistic test stimuli could be created, say, in the form of videos depicting interactions between adults and children, or computer programs that allow CSOs to converse with a "mock child". 
As with Study One, the current findings raise doubts about the notion that CSOs' cognitive distortions are primarily driven by faulty beliefs. Theorists, researchers and clinicians have frequently adopted a stance that essentially equates cognitive distortions with beliefs. Because CSOs commonly articulate or endorse cognitive distortions, this stance has culminated in a widespread perception that the vast majority of CSOs hold beliefs that facilitate their offending. Yet cognitive-experimental research has as yet been unable to find strong evidence that offence-supportive beliefs are held by most CSOs, suggesting that additional mechanisms might underlie cognitive distortions.

In conclusion, the current study adopted a cognitive-experimental approach to study CSOs' offence-supportive beliefs. Contrary to expectations, findings did not support the claim that CSOs hold offence-supportive beliefs that lead them to interpret sentences describing children's actions in sexualised ways. The disjunction between results in the current study and findings from questionnaire and interview studies underscores the need for multiple approaches when seeking understanding of CSOs' beliefs. The results and ensuing discussion contained in this study have also highlighted the need for further research into the phenomena that generate so-called "cognitive distortions". 


\section{Chapter Six}

\section{A Multimethod Study of CSOs' Offence-Supportive Beliefs.}

\section{Introduction}

Results from the two experimental studies discussed in Chapters Four and Five uncovered virtually no evidence that CSOs hold offence-supportive beliefs. As noted, however, the previous experimental designs could have been improved by examining whether CSO participants actually appeared to hold offence-supportive beliefs according to conventional methods of CSO assessment. Measuring offence-supportive beliefs according to interview and questionnaire methods would have allowed comparison of the extent and types of offence-supportive belief endorsement found using conventional measures versus cognitive-experimental methods and thus provided important information about the nature of cognitive distortions. In addition, in the prior two studies when individual ITs were examined CSOs were compared as a group with OCs, despite the fact that Ward and Keenan (1999) posit that not all CSOs endorse all ITs. It is possible that a different pattern of results could have been found by including in the analysis of each IT only those CSOs who appear to support beliefs within that IT according to conventional measures (i.e., questionnaires and interviews). In the current study these two issues are addressed.

The current study also addresses the recommendation made in Studies One and Two that alternative cognitive-experimental techniques be applied to the study of CSOs' offence-supportive beliefs. Hence, a different experimental paradigm called the Rapid Serial Visual Presentation is used in this study. Unlike Study Two, where only Children as Sexual Beings was examined, all five ITs will be investigated to enable examination of the individual beliefs held by each CSO. Also, in accordance with recommendations made in Chapter Five, CSO participants are primed before commencing the cognitive experiment. 
However, unlike the prior study, attempts are made to activate CSOs' offence-supportive beliefs by interviewing them about their offending prior to them taking part in the cognitive experiment. Thus, rather than applying a general, impersonalised prime to CSOs as was done in Study Two (via presentation of visual primes) CSOs are interviewed oneby-one. This ought to allow priming of each CSO's specific beliefs, as each CSO spends time recalling stimuli that played a role in their own offending behaviour.

\section{Study Three: A Multimethod Analysis of Offence-Supportive Beliefs}

In the current study CSOs' and non-sex offenders' beliefs are measured using a cognitive experimental paradigm that has not yet been applied to the sexual offending field and the results compared with data gathered using interview and questionnaire methods. This unique, multimethod design represents the first instance within the sexual offending field where these three methodologies have been used on one participant sample and the results triangulated. In fact, despite the widespread use of questionnaire and interview measures with CSOs, a review of published literature indicates that this study is the first to match and compare the types of beliefs CSOs endorse on both these conventional measures.

The current experimental paradigm, which has previously been called the RSVP task (Rapid Serial Visual Presentation), has often been used to investigate text comprehension processes (see Albrecht \& O'Brien, 1993; Macleod \& Cohen, 1993). RSVP tasks are those in which certain parameters of text presentation are experimentally controlled. Because the technique has traditionally involved presenting single words serially to a fixed location on a viewing screen (Sinclair, Healy, \& Bourne, 1989) the RSVP variant adopted in the current study will be referred to as the RSVP-modified (RSVP-M).

RSVP-M tasks are based on the finding that when individuals read text their sentences reading time varies according to whether the sentence in question continues 
preceding text in a way that is congruent with the mental model constructed by the reader. In short, individuals are faster to read sentences that are consistent with their prior comprehension of the text (Albrecht \& O’Brien, 1993; Galambos, Abelson, \& Black, 1986). Because people use their long-term knowledge structures to interpret texts, the RSVP-M has been used to investigate the content of cognitive structures readers use to comprehend texts (e.g., Macleod \& Cohen, 1993; Wingrove \& Bond, 2005). For instance, Macleod and Cohen used the RSVP-M to investigate the way that students high in trait anxiety comprehended ambiguous texts compared to students low in trait anxiety. All participants read sentence pairs, the first of which could be interpreted in a threatening or nonthreatening manner, and the second of which was unambiguously threatening or nonthreatening. Because individuals high in trait anxiety are thought to hold beliefs that bias them towards threatening interpretations of stimuli (Beck \& Clark, 1988) the authors hypothesised that high-anxiety students would comprehend the ambiguous first sentences as threatening and therefore, relative to low-anxiety students, read unambiguously threatening second sentences faster than nonthreatening sentences. This is exactly the pattern of results that emerged. Similarly, Wingrove and Bond (2005) also presented passages to participants either high or low in trait anger. The authors predicted that, because trait anger is thought to be associated with stable beliefs that encourage hostile interpretations, participants high in trait anger would read texts about potentially angerprovoking situations in a different way to low-anger participants. Wingrove and Bond asked participants to read, sentence by sentence, passages at a self-paced rate that described characters reacting either with anger or non-anger to ambiguous situations. Two key sentences embedded in each passage described these angry or non-angry reactions. As expected, trait anger was associated with faster reading times for key sentences that described an angry reaction versus sentences describing a non-angry reaction. 
In the current experiment CSOs and non-sex offenders read, sentence by sentence, passages that could be interpreted in line with the five belief types hypothesised to characterise CSOs' ITs. Each passage concludes with two sentences that are either consistent or inconsistent with the targeted belief type. It is hypothesised that CSOs will interpret passages in an IT-consistent manner and thus respond faster, relative to controls, to sentences that are consistent with IT content than to sentences that are inconsistent.

CSOs and OCs also answer a questionnaire measure of offence-supportive beliefs that has been found to tap the five ITs outlined in Chapter Two (i.e., the MOLEST; Bumby, 1984; Gannon et al., in press). Recall that the MOLEST has been found by a number of researchers to discriminate between CSOs and non-CSO offender control groups (Arkowitz \& Vess, 2003; Blumenthal et al., 1999; Bumby, 1996; Feelgood et al., 2005; Marshall et al., 2003). In this study the MOLEST is combined with the Balanced Inventory of Desirable Responding (BIDR), which is a measure of tendency to "fake good" designed by Paulhus (1984). In the introduction to this thesis it was explained that questionnaire measures of offence-supportive beliefs are often combined with measures such as the BIDR, purportedly as a way of ensuring that socially desirable responding to offence-supportive belief questionnaires can be monitored and controlled for. Inclusion of the BIDR in this study will allow exploration of the relationship between general socially desirable responding and "faking good" on offence-supportive belief questionnaires. CSOs are also interviewed about their offending and the interviews will be coded for the presence of beliefs relating to each IT. Interviews are conducted prior to the RSVP-M task to ensure that CSOs' offence-supportive beliefs are primed when they complete the RSVP-M.

Results from the three techniques will be compared to see what differences, if any, lie in the information about CSOs' beliefs that they reveal. According to the prevailing theory, CSOs' interviews should identify the presence of offence-supportive beliefs, and 
CSOs should more strongly endorse questionnaire items than nonsexual offender controls. Also, if questionnaires and interviews accurately measure beliefs (rather than other artefacts), the types of beliefs that CSOs endorse in the questionnaire and interview should correlate with the types of beliefs they show evidence of in the RSVP-M study. Thus, this study aims to reveal new and important information about the use of different techniques in the study of CSOs' beliefs.

\section{METHOD}

\section{Participants}

Sixty-seven men took part in this study: 34 CSOs and 33 OCs. Participants were recruited from New Zealand Department of Corrections prisons. Prison officers distributed a recruitment flyer to prisoners inviting them to take part in an "Offender Beliefs study. As with the previous studies, flyers stated that participants were wanted who could read well. Again, volunteer rates in this study could not be monitored. Five CSOs and one OC who took part in this study also participated in Study One. Three CSOs and no OCs who took part in this study participated in Study Two. Selection criteria and demographic details for each participant group follows.

\section{Group selection criteria and demographic details}

CSOS

CSOs were prison inmates whose index offences included at least one sexual conviction against a person under the age of 16 . Their ages ranged from 20 to 70 years $(M=46.35$, $S D=11.52)$ and their victims' ages ranged from two to 16 years $(M=10.06, S D=3.29)$. According to CSOs' conviction records, 25 were extrafamilial offenders and nine were intrafamilial. Four extrafamilial and one intrafamilial CSOs had received cognitivebehavioural therapy to reconstruct offence-related cognitions but had re-offended against a child since. Index and historic conviction records revealed that seven CSOs had sexually assaulted a person over the age of 16. The mean Static-99 score for all CSO participants 
indicated a medium recidivism risk level $(M=3.53, S D=2.60)$ according to the criteria set by Phenix et al. (2000). Numbers of years CSOs had spent in formal education ranged from ten to 17 years $(M=11.15, S D=2.12)$. Fourteen CSOs identified themselves as Māori, 19 as European, and one as "Other".

OCs were prison inmates who had never been convicted of a sexual offence. They were aged from 20 to 50 years $(M=31.27, S D=7.39)$ and had spent between ten and 17 years in formal education $(M=10.93, S D=1.50)$. Thirteen OCs identified themselves as Māori, 19 as European, and one as "Other". Independent sample t-tests revealed the two offender groups did not differ significantly on number of years spent in education, number of violent convictions, or total number of convictions. CSOs and OCs differed significantly on age, $t(65)=6.36, p<.001$, with CSOs having a higher mean age $(M=$ 46.35, $S D=11.52)$ than OCs $(M=31.27, S D=7.39)$. Characteristics of the participant groups are displayed in Table 6.1. 
Table 6.1.

Demographic details for participant groups: Study Three

\begin{tabular}{|c|c|c|}
\hline & $\begin{array}{c}\text { Child Sex Offenders } \\
n=34\end{array}$ & $\begin{array}{c}\text { Offender Controls } \\
n=33\end{array}$ \\
\hline \multicolumn{3}{|l|}{ Age (years) } \\
\hline Mean & $46.35 * * *$ & 31.27 \\
\hline$S D$ & 11.52 & 7.39 \\
\hline \multicolumn{3}{|c|}{ Years in education } \\
\hline Mean & 11.15 & 10.93 \\
\hline$S D$ & 2.12 & 1.50 \\
\hline \multicolumn{3}{|l|}{ Ethnicity (n) } \\
\hline Maori & 14 & 13 \\
\hline European & 19 & 19 \\
\hline Other & 1 & 1 \\
\hline \multicolumn{3}{|c|}{ Total Convictions (n) } \\
\hline Mean & 29.79 & 47.76 \\
\hline$S D$ & 35.10 & 77.57 \\
\hline \multicolumn{3}{|c|}{ Violent Convictions (n) } \\
\hline Mean & 2.76 & 1.70 \\
\hline$S D$ & 6.04 & 1.90 \\
\hline \multicolumn{3}{|l|}{ CSO type (n) } \\
\hline Intra & 9.0 & - \\
\hline Extrafamilial & 25.0 & - \\
\hline \multicolumn{3}{|c|}{ Child vicitms (n) } \\
\hline Mean & 3.88 & - \\
\hline$S D$ & 5.53 & - \\
\hline \multicolumn{3}{|c|}{ Victim age (years) } \\
\hline Mean & 10.06 & - \\
\hline$S D$ & 3.29 & - \\
\hline \multicolumn{3}{|c|}{ STATIC-99 Score } \\
\hline Mean & 3.53 & - \\
\hline$S D$ & 2.60 & - \\
\hline
\end{tabular}




\section{Ethical Considerations}

The procedures and materials used in this study were approved by New Zealand Department of Corrections and Victoria University of Wellington. Before testing began all participants read (or were read) an information sheet outlining the procedure and their rights as participants (which followed NZPS Code of Ethics criteria). Participants were told they would need to provide details of their offending, which would be checked against their prison records on completion of testing. CSO participants were also informed that their interviews would be audiotaped and transcribed. Participants signed a consent form before taking part in the experiment and were sent a combined debriefing and results summary sheet once results were collated. Participants were also provided with a feedback form regarding the study, on which they could indicate their thoughts about the research and mention any issues that may have arisen through their participation.

\section{Overall Design}

The research design utilised three methods, as depicted in Figure 6.1. CSOs were interviewed and their transcripts used for a within-subjects analysis. CSOs and OCs took part in the RSVP-M and then completed a cognitive distortion questionnaire. 


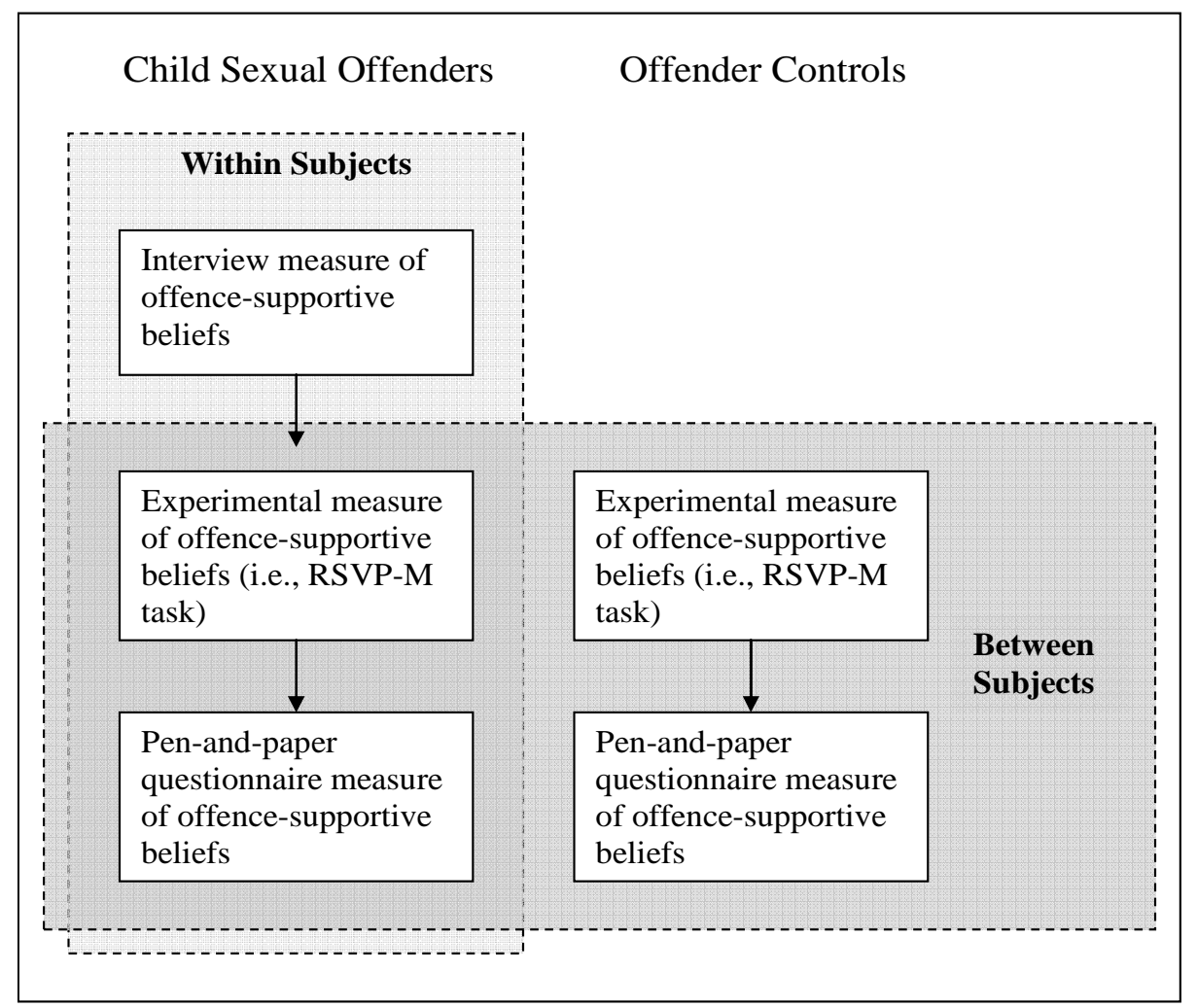

Figure 6.1. Study Three Research Design Overview

\section{Measures}

\section{Measure One-Interview}

A semi-structured interview was conducted to measure CSOs' offence-supportive beliefs (see Appendix Seven) and to activate those beliefs prior to CSOs commencing the RSVP-M. Open-ended interview questions were drawn from various sources, including interview schedules used to identify ITs in other CSO studies (e.g., Marziano, Ward, Beech, \& Pattison, 2006) and consultation with Tony Ward and with the directors of New Zealand's two CSO treatment programmes (Te Piriti and Kia Marama). Questions were designed to elicit beliefs that could be classified according to Ward and Keenan's (1999) IT categories. Broadly, CSOs were asked to explain events leading up to their offences, 
their thoughts and feelings at the time of the offending, and their and their victims' reactions to the offending. Four CSOs who denied their crimes were asked an unstructured series of questions regarding their perception of the offence (e.g., "why do you think the child accused you?" and "how was your relationship with the child different from other relationships?").

\section{Measure Two-RSVP-M}

This design was based upon the successful RSVP-M design utilised by Wingrove and Bond (2005) wherein participants read short passages, sentence by sentence, at a selfpaced rate, while time taken to respond to each sentence was recorded. In the current study participants read 28 passages containing six to nine sentences (see Appendix Ten). Eight passages were "fillers" added to obscure task aims and provide a measure of overall reading speed. Twenty passages were constructed to tap into the five IT types. Embedded within these 20 passages were 40 target sentences (i.e. 20 sentence pairs) half of which were IT-consistent, and half of which were IT-inconsistent. To ensure a balanced design two versions of RSVP-M task material were created such that for every consistent target sentence pair that appeared in a passage in version one, an inconsistent target sentence pair appeared in that passage in version two, and vice versa. (see Figure 2). Participants were randomly assigned to each version of the RSVP-M. Target sentences were between six and nine words long. Target sentence pairs were carefully balanced for length and FleschKincaid Reading Ease levels across versions and IT type. To disguise task aims, each passage was followed by a related question which participants were instructed to answer yes or no to. For example, a passage about a lonely widowed man who molested his daughter was followed by the question, "Should David be punished for his actions?" Participants were told the study aim was to see whether different offender groups answered these questions differently. 
IT

Example Passage

Children Derek was at his girlfriend's house.

as Sexual He was babysitting his girlfriend's daughter, Jane.

Beings Jane was sitting beside him on the couch, watching TV. She snuggled up to him and put her hand on his leg.

Dangerous

World

Entitlement

Nature of

John was being taken to court

Harm

He knew he would probably go to jail

He had gotten in trouble for groping a kid's bottom

He hadri't done anything else to the child.

Uncontrollable

lan woke up with a terrible hangover

He had fallen asleep in his friend's spare bed

His friend's daughter was asleep in the other bed.

$\mathrm{He}$ groaned as he remembered the night before.

He had tried to kiss and grope his friend's daughter as she lay in bed
Target Sentences

(Varied across two versions)

\section{IT-consistent target sentences}

Derek knew she was aroused.

She was clearlv flirting with him.

or

IT-inconsistent target sentences

Jane was a girl who loved cuddles She was such a friendly child.

\section{IT-consistent target sentences}

It was much safer being friends with children. You could rely on children more than adults.

or

$\underline{\text { IT-inconsistent target sentences }}$

He knew he needed some adult friends too.

You can't rely on children as your only friends.

\section{IT-consistent target sentences}

Steve was furious that she said no to him.

or She should always do what her father said.

\section{$\underline{\text { IT-inconsistent target sentences }}$}

Steve knew that he couldn't go in there.

She had a right to be alone in there.

\section{IT-consistent target sentences}

He shouldn't be charged for such a small thing.

How could one little touch do any harm?

or IT-inconsistent target sentences

- But he knew one touch could damage a child.

It wasn't right to harm a child like that.

\section{IT-consistent target sentences}

In some ways it wasn't his fault.

The alcohol had made him do it.

or

IT-inconsistent target sentences

There was no excuse for doing that to her. He should have made himself stop.

Figure 6.2. RSVP-M Design 
The RSVP-M task was programmed using a Superlab package and run on a 12 inch colour monitor screen (resolution $=1440 \times 900$ pixels) from a Dell Latitude D620 laptop computer. Written instructions and stimuli were centred on a white screen in black 20 pt. Tahoma font. Participants used a Cedrus Model RB-730 response pad with a clearly labelled, centrally positioned key to view each new sentence. Follow-up question answers were also indicated using the two endmost keys of the response pad, which were labelled Yes and No. These two keys were not alternated across participants because question responses were not a variable of interest in this study. Superlab controlled the random presentation of 28 passages and recorded (in milliseconds) the time that elapsed between each button press participants made to view a new sentence.

\section{Measure Three - Questionnaire}

The 78-item questionnaire was comprised of two measures (see Appendix Eight):

1) MOLEST; Bumby, 1996.

The MOLEST is a 38-item questionnaire designed to measure offence-supportive beliefs. It was selected for use in the current study because of its demonstrated success in distinguishing between CSOs and other offender groups (e.g., Arkowitz \& Vess, 2003; Blumenthal et al., 1999; Bumby, 1996; Feelgood, Cortini \& Thompson, 2005, Pervan \& Hunter, 2007). Bumby (1996) reported that psychometric properties of the MOLEST are excellent (internal consistency; $\alpha=.97$ and test-retest reliability; $r=.84$ ). An example item is, "Some children can act very seductively". Items are scored on a 4-point Likert scale from 1 (Strongly disagree) to 4 (Strongly agree) and the scores are summed. In line with previous research (Gannon, et al., 2007) and to increase measure sensitivity the MOLEST's response format was modified by adding unsure as a response option to create a 5-point scale. Gannon et al. (in press) found that the MOLEST measured each of the five belief clusters identified by Ward and Keenan (1999). The ITs most frequently represented in the MOLEST are Children as Sexual Beings (15 items), Nature of Harm (10 items), and 
Uncontrollable (five items). Unfortunately, Dangerous World and Entitlement are not well represented on the MOLEST: these ITs were tapped by only three and two MOLEST items respectively. The IT categories to which MOLEST items were assigned by Gannon et al. (2007) are listed in Appendix Nine.

2) Balanced Inventory of Desirable Responding (BIDR); Paulhus, 1984, 1988.

To measure whether participants responded to the MOLEST in a socially desirable way items were randomly mixed with the 40-item BIDR. The BIDR is a self-report measure designed to assess the general tendency to answer questionnaire items in a socially desirable manner. It was chosen in the current study because it is a recommended measure of biased responding that is commonly used in conjunction with forensic risk assessment measures (Kroner \& Weekes, 1996; Lanyon, 2001). Items on the original measure were scored on a 7-point Likert scale ranging from 1 (not true) to 7 (very true). However, in line with previous research (Gannon et al., 2007), in this study a 5-point scale was used in place of Paulhus' 7-point one. This modification to the scale was made for two reasons: 1) researchers in the current study have previously observed that the complexity involved in answering a seven-point scale can be confusing for offender participants, and, 2) adopting a five-point scale allowed items from this measure to be randomly mixed with MOLEST items and thereby helped disguise the MOLEST's purpose. Thus, in the current study, scale ratings ranged from 1 (not true) to 5 (very true). In the BIDR half the items are reverse scored. For reverse-scored items a rating of 5, for instance, was assigned a score of 1 , while a rating of 1 was assigned a score of 5 . The scores were then summed and correlated with the MOLEST ${ }^{5}$.

\footnotetext{
5 This use of the BIDR differs from that recommended by Paulhus (1988), who suggests removing participants with extreme responses (i.e., less than one or greater than 12 Likert scale responses of six or seven). In the current study, BIDR items were correlated with MOLEST items because a correlational approach has been adopted by other researchers. That is, other researchers have ensured that measures such as the Marlowe-Crowne Socially Desirability Scale (see Bumby, 1996; Hayashino et al., 1995; Stermac \& Segal, 1989) and Other-Deception and Self-Deception Questionnaires (see Blumenthal et al., 1999) do not correlate (or are added as covariates) with CSO cognition scale responses. Nevertheless, the BIDR was not an ideal scale to use for correlational analysis in this study. Fortunately, RSVP-M results remained unchanged when the statistical analyses described in this study were rerun with extreme BIDR scorers removed from the sample.
} 
The BIDR has two subscales: Impression Management scale (IM) and the Self Deception scale (SD). The IM contains 20 items measuring overt attempts to appear favourable to others (e.g., "I don't gossip about other people's business"). Paulhus (1988) reported good IM internal consistency ( $\alpha$ ranging from .75 to .86 ), and moderate test-retest reliability $(r=.65)$ over five weeks. The SD contains 20 items measuring participants' tendency to self deceive (e.g., "I am a completely rational person"). Paulhus reported good SD internal consistency ( $\alpha$ ranging from .68 to .80 ) and test-retest reliability $(r=.69$ ) over five weeks.

Certain questionnaire items pertained to events some participants had not experienced and so could not be answered by those participants (e.g., "I always declare everything at customs"). These null responses were excluded from the group means reported below.

\section{Procedure}

Participants were tested individually in a single session that took between 40 and 120 minutes. After giving written consent, participants provided demographic information (e.g., age and ethnicity) and outlined their index and historical convictions. CSOs then completed steps one, two and three below, while OCs completed steps two and three only. Step One - Interview

CSOs were interviewed for between 20 and 60 minutes regarding their sexual offending. Each interview was audiotaped and later transcribed.

Step Two - RSVP-M

After receiving standardised verbal instructions participants completed a practice trial involving one passage and follow-up question. Participants then read the experimental passages at a self-paced rate and answered the question that followed each passage.

Step Three - Questionnaire 
Participants were given a paper questionnaire sheet to answer on their own. Responses were made by ticking a box on the Likert scale printed beneath each item.

\section{RESULTS}

\section{Interview Data}

Interview transcripts were analysed and coded for content that endorsed IT themes ${ }^{6}$. Content that seemed IT-consistent was highlighted and labelled. Coders noted instances where they were uncertain whether the content could definitely be categorised as IT-consistent. They then reviewed each transcript and considered whether, on balance, the speaker showed clear signs of holding each IT. To be classed as holding a particular IT the speaker must have uttered one or more statements that the coder was certain endorsed the IT.

The main coder (Rater One) was the principle researcher in this study. Initially, the research supervisor (TW) held a training session with Rater One and reviewed three transcripts Rater One had analysed and coded. There was $100 \%$ agreement between TW and Rater One regarding IT-consistent themes in these transcripts. To allow reliability checks a second coder (Rater Two) who had coding experience and familiarity with ITs was drawn from Victoria University of Wellington's Clinical Diploma programme. Rater One trained Rater Two using five randomly-selected transcripts. Raters One and Two then independently coded $40 \%$ of the total transcripts. Rater One coded the remaining $36 \%$ of transcripts. Interrater reliability was examined using Cohen's Kappa. Because each IT was coded as present or absent there were five possible points of disagreement. For Children as Sexual Beings agreement occurred in $100 \%$ of ratings $(\kappa=1.00, T=3.61, p<.001)$. For Nature of Harm there was $100 \%$ agreement $(\kappa=1.00 ., T=3.60, p<.001)$ and for Uncontrollable $91 \%$ agreement $(\kappa=.52, T=2.13, p=.033)$. For Dangerous World there

\footnotetext{
${ }^{6}$ One participant who strongly denied his offending declined to be interviewed.
} 
was $85 \%$ agreement $(\kappa=.68, T=2.60, p=.009)$ and for Entitlement $100 \%$ agreement $(\kappa$ $=1.00 ., T=3.61, p<.001)$. According to Fliess' (1981) criteria, the reliability rating for Uncontrollable was "fair," for Dangerous World it was "good", and for Children as Sexual Beings, Nature of Harm, and Entitlement ratings were “excellent." Disagreements over ratings were discussed and resolved.

Thirty-two interviews were found to contain one or more IT-consistent themes, while one interview (where the speaker strongly denied offending) held no such themes. Numbers of CSOs endorsing each IT are as follows: Children as Sexual Beings, $n=25$; Nature of Harm, $n=24$; Uncontrollable, $n=25$; Dangerous World, $n=14$; Entitlement, $n$ $=4$.

\section{Questionnaire Analysis}

\section{Cognitive distortions}

Based on prior research, it was hypothesised that CSO participants in the current study would tend to agree more with cognitive distortion questionnaire items than offender controls. Each participant's mean response score across 38 MOLEST items was calculated: these could range from 1 (strongly disagree) to 5 (strongly agree). Independent-samples t-tests comparing CSOs' and OCs' responses showed the two groups significantly differed in their responses, $t(65)=3.92, p<.001$, partial eta squared $=.191$, with CSOs indicating greater endorsement of items $(M=2.30, S D=.58)$ than OCs $(M=$ $1.84, S D=.34)$. Both groups' average scores tended towards the disagree option, but CSOs' scores fell between disagree and unsure, while OCs' scores fell between disagree and strongly disagree. A Pearson correlation was not found between CSOs' Static-99 scores and their MOLEST scores, $r=-.147, n=34, \mathrm{p}=.407$.

Questionnaire items were grouped according to the IT they tapped and independent-samples t-tests were run for three ITs: Children as Sexual Beings (15 items), Nature of Harm (10 items), and Uncontrollable (five items). Dangerous World and 
Entitlement were excluded from the analysis for two reasons: 1) Offenders and delinquents other than CSOs are thought to hold cognitions that have themes of entitlement (Walters, 1995) and of the world being a dangerous, hostile place (Beck, 1999; Palmer, 2000; Walters, 2002), and 2) these ITs were tapped by only three and two items respectively.

Because the analysis involved three t-tests, alpha was set at .01 to compensate for the associated inflation of type 1 error. CSOs' scores were significantly higher for Children as Sexual Beings, $t(65)=3.01, p=.004$, partial eta squared $=.122,(M=2.35$, $S D=0.68$ for CSOs and $M=1.94, S D=0.39$ for OCs), Nature of Harm, $t(65)=2.99, p=$ .004 , partial eta squared $=.121,(M=1.92, S D=0.62$ for CSOs and $M=1.53, S D=0.41$ for OCs $)$, and Uncontrollable, $t(65)=4.50, p<.001$, partial eta squared $=.237, \quad(M=$ 2.85, $S D=0.70$ for CSOs and $M=2.11, S D=0.64$ for OCs). Thus-relative to OCsCSOs made significantly higher endorsements of statements depicting (1) children as sexual, (2) children as being unharmed by sexual abuse, and (3) sexual abuse as uncontrollable.

\section{Self Deception (SD) and Impression Management (IM)}

As with the MOLEST, individual average response scores were calculated for SD and IM scales. Again, mean scores could range from 1 (strongly disagree) to 5 (strongly agree). As expected, no significant difference was found in SD responses, $t(65)=-0.52, p$ $=.604$. Both groups answered unsure to SD items $(M=3.00, S D=0.39$ for CSOs and $M=$ 3.05, $S D=0.32$ for OCs). However, an unexpected significant difference was found between CSOs' and offender controls' IM scores, $t(65)=4.43, p<.001$, with CSOs indicating greater endorsement of items $(M=2.79, S D=0.52)$ than OCs $(M=2.27, S D=$ 0.43). Both groups' scores fell between the disagree and unsure options, but CSOs' scores fell closer to unsure, while offender controls' scores fell closer to disagree. Because groups differed on IM, Pearson correlations were calculated to investigate whether IM scores might account for group differences on the MOLEST. No significant correlation 
existed between CSOs' MOLEST and IM scores, $r=-.076, n=34, \mathrm{p}=.668$, nor between OCs' MOLEST and IM scores, $r=.220, n=33, \mathrm{p}=.219$. Also, when a one-way ANOVA was run with IM as a covariate virtually no shift occurred in the MOLEST responses of CSOs $(M=2.29)$ and OCs $(M=1.85)$, and CSOs still showed significantly higher levels of MOLEST endorsement, $t(64)=2.54, p=.001$. A Pearson correlation was also calculated to investigate the relationship between SD and the MOLEST. Again, no significant correlations were found between CSOs' SD and MOLEST scores, $r=-.300, n$ $=34, \mathrm{p}=.085$, and OCs' SD and MOLEST scores, $r=-.280, n=33, \mathrm{p}=.115$.

In summary, questionnaire responses indicated that CSOs endorsed cognitive distortion items more strongly than offender controls, although they still tended to disagree with items. Although CSOs' response scores on the SD scale were statistically equivalent with offender controls', their response scores on the IM scale indicated higher levels of impression management than offender controls. However, neither CSOs nor OCs showed correlations between their IM and MOLEST scores, suggesting that CSOs' higher MOLEST scores (and OCs' lower MOLEST scores) were not influenced by a general tendency to portray oneself in a more favourable light. Results from the RSVP-M will also shed light on whether CSOs deliberately attempted to hide distorted beliefs when answering the MOLEST since this cognitive-experimental task examines relatively unconscious, automatic responses.

\section{RSVP-M}

Before comparing CSOs' and offender controls' responses to target sentences it was important to ensure general sentence response times did not differ. Individual mean RTs to all filler passage sentences combined were calculated. Independent-samples t-tests indicated that there was no significant difference $t(65)=-0.19, p=.850$, in the mean time CSOs $(M=16611.51 \mathrm{~ms}, S D=7852.41 \mathrm{~ms})$ and $\mathrm{OCs}(M=16946.31 \mathrm{~ms}, S D=6477.31 \mathrm{~ms})$ took to respond to filler passage sentences. 
As discussed in Chapter Four, RT data typically contains extremely long or short responses resulting from factors unrelated to the variable being measured (Ratcliffe, 1993). For example, very short RTs can reflect accidental button-pressing while very long RTs result when participants are distracted from the task. In line with recommendations for dealing with such data (Barnett \& Lewis, 1978; Ratcliffe, 1993), RTs to target sentences were Windsorised such that CSOs' and OCs' extreme RTs were adjusted to sit within two standard deviations of their group's mean.

Next, the important question of whether CSOs and OCs showed different patterns of responding to IT-consistent and IT-inconsistent target sentences was explored. If CSOs hold IT-consistent beliefs, when compared to controls they should show a pattern of smaller RTs to IT-consistent than IT-inconsistent sentences. To investigate, each participant's mean RTs to IT-consistent and IT-inconsistent target sentences within each IT were calculated. Next, these means were used to deduce the pattern of responding each participant showed within the five IT categories. Within each IT category IT-consistent sentences were subtracted from IT-inconsistent sentences to produce a mean difference score. In addition, mean RTs to all IT-consistent sentences were subtracted from all ITinconsistent sentences to produce a mean difference score for all ITs combined. A positive mean difference score would indicate a participant was faster to respond to IT-consistent sentences relative to IT-inconsistent sentences, and thus would indicate a more "distorted" pattern of responding.

A series of alpha adjusted (.01) independent-samples t-tests were run to investigate whether CSOs and OCs differed in their pattern of responding. Surprisingly, no significant differences were found in the mean difference scores for CSOs and OCs for Children as Sexual Beings, $t(65)=-0.79, p=.430$, partial eta squared $=.010\left(\right.$ CSOs' $^{\prime} M=-564.34 \mathrm{~ms}$, $S D=5567.10 \mathrm{~ms} ;$ OCs' $M=581.38 \mathrm{~ms}, S D=6227.47 \mathrm{~ms})$, Dangerous World,$t(65)=-$ $0.02, p=.980$, partial eta squared $<.001$ (CSOs' $M=-203.04 \mathrm{~ms}, S D=10069.91 \mathrm{~ms}$; 
OCs' $M=-156.30 \mathrm{~ms}, S D=4325.66 \mathrm{~ms})$, and Entitlement, $t(65)=-0.02, p=.980$, partial eta squared $<.001$ (CSOs' $M=-2123.92 \mathrm{~ms}, S D=7671.96 \mathrm{~ms}$; OCs' $M=-2069.05 \mathrm{~ms}, S D$ $=10335.04 \mathrm{~ms})$. Similarly, no significant differences were found for Nature of Harm, $t(65)$ $=-0.14, p=.885$, partial eta squared $<.001\left(\right.$ CSOs' $^{\prime} M=-2851.19 \mathrm{~ms}, S D=9304.19 \mathrm{~ms}$; OCs' $M=-2565.22 \mathrm{~ms}, S D=6612.44 \mathrm{~ms})$, Uncontrollable, $t(65)=-0.30, p=.764$, partial eta squared $=.001\left(\right.$ CSOs' $^{\prime} M=321.71 \mathrm{~ms}, S D=10926.20 \mathrm{~ms}$; OCs' $M=972.13 \mathrm{~ms}, S D=$ $5876.43 \mathrm{~ms}$ ), nor for all ITs combined, $t(65)=-0.57, p=.574$, partial eta squared $=.005$ (CSOs' $M=-1084.16 \mathrm{~ms}, S D=3213.97 \mathrm{~ms}$; OCs' $M=-647.41 \mathrm{~ms}, S D=3108.09 \mathrm{~ms}$ ). A Pearson correlation was not found between CSOs' Static-99 scores and their RSVP responses to any IT.

Thus far no significant differences could be found between CSOs and offender controls. However, each CSO would not be expected to hold all five IT-themed belief clusters. Inclusion of CSOs who do not appear to endorse a particular IT when responding to conventional assessments may therefore have obscured group differences in RSVP-M responses to that particular IT. Hence, data gathered from the interviews and questionnaire was now used to partition out CSOs from the RSVP-M analysis who showed no evidence of holding each IT according to these conventional methods.

For this phase of the analysis only three ITs were investigated: Children as Sexual Beings, Nature of Harm, and Uncontrollable. Again, this was done because Dangerous World and Entitlement beliefs are thought to be held by offenders other than CSOs and because these ITs achieved low levels of endorsement in the interviews and were underrepresented by items in the MOLEST.

To prepare RSVP-M data for this phase of the analysis, two datasets were created for each of the three ITs under investigation. Of the two datasets, one had removed from it all the CSOs who did not endorse that particular IT in their interview. The other had removed from it all CSOs who did not endorse that particular IT on the questionnaire (i.e., 
CSOs whose mean Likert scale scores across questionnaire items tapping that IT were equal to or lower than the mean OC score for that IT). Numbers of CSOs classified as endorsing each of the three ITs on the questionnaire were: Children as Sexual Beings, $\mathrm{n}=$ 20; Nature of Harm, $\mathrm{n}=20$; Uncontrollable, $\mathrm{n}=13$. To recap results reported earlier, numbers of CSOs endorsing ITs in the interview were: Children as Sexual Beings, $\mathrm{n}=25$; Nature of Harm, $\mathrm{n}=24 ;$ Uncontrollable, $\mathrm{n}=25$.

Having selected CSOs who, according to traditional measures, appeared to hold distorted beliefs, would RSVP-M data reveal differences between CSO and OC responses? A series of alpha adjusted (.01) independent-samples t-tests were rerun on each of the six datasets to see if a difference existed between the group's mean difference scores for each of the three ITs. Looking at datasets where CSOs had been selected for removal according to interview responses, no significant differences were found in the mean difference scores for CSOs and OCs for Children as Sexual Beings, $t(56)=-0.60, p$ $=.552$, partial eta squared $=.006\left(\right.$ CSOs' $^{\prime} M=-408.24 \mathrm{~ms}, S D=6249.75 \mathrm{~ms} ;$ OCs' $M=$ $581.38 \mathrm{~ms}, S D=6227.47 \mathrm{~ms})$, Nature of Harm, $t(52)=-0.20, p=.843$, partial eta squared $=.001\left(\right.$ CSOs' $^{\prime} M=-3021.44 \mathrm{~ms}, S D=10244.03 \mathrm{~ms}$; OCs' $M=-2565.22 \mathrm{~ms}, S D=$ $6612.44 \mathrm{~ms})$, and Uncontrollable, $t(56)=0.16, p=.875$, partial eta squared $<.001$ (CSOs' $M=1349.70 \mathrm{~ms}, S D=11961.92 \mathrm{~ms} ;$ OCs' $M=972.13 \mathrm{~ms}, S D=5876.43 \mathrm{~ms})$.

Looking at datasets where CSOs had been removed according to their questionnaire responses, again no significant differences were found for mean difference scores for Children as Sexual Beings, $t(53)=-1.23, p=.224$, partial eta squared $=.028$ (CSOs' $M=-1490.80 \mathrm{~ms}, S D=5950.92 \mathrm{~ms}$; OCs' $M=581.38 \mathrm{~ms}, S D=6227.47 \mathrm{~ms}$ ), Nature of Harm, $t(55)=-0.81, p=.421$, partial eta squared $=.012\left(\right.$ CSOs' $^{M}=-$ $4326.59 \mathrm{~ms}, \quad S D=9804.02 \mathrm{~ms} ;$ OCs' $M=-2565.22 \mathrm{~ms}, \quad S D=6612.44 \mathrm{~ms})$, and Uncontrollable, $t(61)=-0.42, p=.674$, partial eta squared $=.003\left(\right.$ CSOs' $^{\prime} M=29.33 \mathrm{~ms}$, $S D=11225.12 \mathrm{~ms} ;$ OCs' $M=972.13 \mathrm{~ms}, S D=5876.43 \mathrm{~ms})$. 
Because CSOs and OCs significantly differed on age all questionnaire and RSVP-M analyses mentioned above were rerun as ANOVAs with age added as a covariate. In each case the pattern of results remained the same.

\section{Agreement Across Measures}

An important final question to address concerns the extent to which interview, questionnaire and RSVP-M responses related to each other. In other words, did the three measures show agreement in terms of whether they classified a CSO as holding a particular IT? To examine this, for each measure CSOs were assigned a ' 1 ' if they had been classed as supporting a particular IT and a ' 0 ' if they had not. CSOs were classed as supporting an IT on the RSVP-M if their mean difference score for that IT was positive (see above for interview and questionnaire classification methods). Classification of IT endorsement according to the three methods is shown in Table 6.2. Cohen's Kappa was then calculated to assess agreement between the three measures as to whether each CSO held each IT. Once again, only responses to Children as Sexual Beings, Nature of Harm, and Uncontrollable were tested. Table 6.3 sets out the percentage of CSOs endorsing each belief cluster, while Table 6.4 provides interrater reliability coefficients between belief clusters.

Kappa ratings below .40 are considered "poor" (Fliess, 1981), while negative Kappa ratings indicate observed agreement is less than chance agreement. As Table 6.3 demonstrates, interrater reliability between the three measures was very low, ranging from poor to below chance levels. The interview and questionnaire measures were similar in terms of the numbers of CSOs that were classed as holding a particular IT. However, the two measures disagreed in terms of which CSOs were classed as holding each IT. In other words, the types of IT a CSO was demonstrated to hold depended on the type of measure used. Compared to the two conventional measures, the RSVP-M classed far fewer CSOs as holding each of the three ITs (RSVP-M classification rates ranged from about one third 
to one half lower than interview and questionnaire rates). Therefore, the lack of agreement between the RSVP-M and other measures could be due to low levels of RSVP-M IT endorsement, although perusal of the data in Table 6.2 suggests that the RSVP-M varied from other measures both in terms of the number and type of ITs being measured. 
Table 6.2

Classification of IT endorsement, per individual, according to three methods

\begin{tabular}{|c|c|c|c|c|c|c|c|c|c|}
\hline \multirow[b]{2}{*}{ CSO } & \multicolumn{3}{|c|}{ Children as Sexual Beings } & \multicolumn{3}{|c|}{ Nature of Harm } & \multicolumn{3}{|c|}{ Uncontrollable } \\
\hline & Interview & Questionnaire & RSVP-M & Interview & Questionnaire & RSVP-M & Interview & Questionnaire & RSVP-M \\
\hline 1 & $\mathrm{x}$ & x & X & & x & & & $\mathrm{x}$ & \\
\hline 2 & $x$ & $x$ & & & $x$ & $\mathrm{x}$ & $\mathrm{x}$ & $x$ & $\mathrm{X}$ \\
\hline 3 & $\mathrm{x}$ & $\mathrm{x}$ & & $\mathrm{X}$ & $\mathrm{x}$ & $\mathrm{x}$ & $\mathrm{x}$ & $x$ & $\mathrm{x}$ \\
\hline 4 & $x$ & $x$ & & & $x$ & & & $x$ & $x$ \\
\hline 5 & $\mathrm{x}$ & $\mathrm{x}$ & $\mathrm{x}$ & $\mathrm{x}$ & $\mathrm{x}$ & & & $\mathrm{x}$ & \\
\hline 6 & $\mathrm{x}$ & $\mathrm{x}$ & $\mathrm{x}$ & & $\mathrm{x}$ & & $\mathrm{x}$ & $x$ & \\
\hline 7 & $\mathrm{x}$ & $\mathrm{x}$ & $\mathrm{X}$ & $\mathrm{x}$ & $\mathrm{x}$ & & $\mathrm{x}$ & $\mathrm{x}$ & \\
\hline 8 & & $\mathrm{x}$ & & & $\mathrm{x}$ & & $\mathrm{x}$ & $\mathrm{x}$ & $\mathrm{x}$ \\
\hline 9 & $\mathrm{x}$ & $\mathrm{x}$ & $\mathrm{x}$ & & $\mathrm{x}$ & & $\mathrm{x}$ & $\mathrm{x}$ & \\
\hline 10 & $x$ & & & $\mathrm{x}$ & & & $x$ & & \\
\hline 11 & $\mathrm{x}$ & & $\mathrm{x}$ & $\mathrm{x}$ & & & $\mathrm{x}$ & $\mathrm{x}$ & \\
\hline 12 & & $\mathrm{x}$ & $\mathrm{x}$ & & $\mathrm{x}$ & & & $\mathrm{x}$ & \\
\hline 13 & $\mathrm{x}$ & & & & & & & $\mathrm{x}$ & \\
\hline 14 & $\mathrm{x}$ & & $\mathrm{X}$ & $\mathrm{x}$ & & & $\mathrm{x}$ & $\mathrm{x}$ & \\
\hline 15 & $\mathrm{x}$ & $\mathrm{x}$ & $\mathrm{x}$ & $\mathrm{x}$ & $\mathrm{x}$ & $\mathrm{x}$ & $\mathrm{x}$ & $\mathrm{x}$ & \\
\hline 16 & $\mathrm{x}$ & & & $\mathrm{x}$ & $x$ & $\mathrm{x}$ & $x$ & $\mathrm{x}$ & $\mathrm{x}$ \\
\hline 17 & & $\mathrm{X}$ & & & $\mathrm{x}$ & $\mathrm{x}$ & $\mathrm{x}$ & $\mathrm{x}$ & $\mathrm{X}$ \\
\hline 18 & & & & $\mathrm{x}$ & & $x$ & & $x$ & $x$ \\
\hline 19 & $\mathrm{x}$ & $\mathrm{x}$ & & $\mathrm{x}$ & $x$ & $x$ & $X$ & $X$ & $X$ \\
\hline 20 & $x$ & $x$ & & $x$ & $x$ & & $x$ & $x$ & $X$ \\
\hline 21 & & $x$ & $x$ & $x$ & & & $x$ & & $x$ \\
\hline 22 & $X$ & $x$ & & $x$ & $x$ & $x$ & $x$ & $x$ & $\mathrm{X}$ \\
\hline 23 & $x$ & $x$ & & $x$ & $x$ & & $x$ & $x$ & $x$ \\
\hline 24 & & & & & $x$ & $x$ & $x$ & & $x$ \\
\hline 25 & & $x$ & & $X$ & $x$ & & $x$ & $x$ & \\
\hline 26 & $x$ & & $x$ & $x$ & $x$ & & $x$ & & \\
\hline 27 & $x$ & & $x$ & $x$ & $x$ & $x$ & $x$ & $x$ & \\
\hline 28 & $x$ & $x$ & $x$ & $x$ & $x$ & & $x$ & $x$ & \\
\hline 29 & $x$ & $x$ & $x$ & & $x$ & $x$ & & $x$ & \\
\hline 30 & $X$ & $X$ & $X$ & $X$ & & & $x$ & $X$ & \\
\hline 31 & $x$ & & & $x$ & & $x$ & $x$ & $x$ & \\
\hline 32 & $x$ & $X$ & & $X$ & $X$ & $X$ & & $x$ & $x$ \\
\hline 33 & & & & & & $x$ & $x$ & $x$ & $x$ \\
\hline Total & 25 & 22 & 15 & 21 & 24 & 14 & 25 & 29 & 15 \\
\hline
\end{tabular}


Table 6.3

Percentages of CSOs classed as holding three belief clusters by measure $(n=33)$

\begin{tabular}{lccc}
\hline & Interview & Questionnaire & RSVP-M \\
\hline IT & Percentage Classified & \\
\hline $\begin{array}{l}\text { Children as Sexual } \\
\text { Beings (CSB) }\end{array}$ & $76 \%$ & $67 \%$ & $45 \%$ \\
$\begin{array}{l}\text { Nature of Harm } \\
\text { (NOH) }\end{array}$ & $64 \%$ & $73 \%$ & $48 \%$ \\
$\begin{array}{l}\text { Uncontrollable } \\
\text { (UNC) }\end{array}$ & $76 \%$ & $91 \%$ & $48 \%$ \\
\hline
\end{tabular}

$\underline{\text { Table } 6.4}$

Interrater reliability between measure classifications of CSOs' belief clusters

\begin{tabular}{llll}
\hline IT & & \multicolumn{2}{c}{ Agreement } \\
\hline \multicolumn{1}{l}{$\begin{array}{l}\text { Measure } \\
\text { Sexualdren as }\end{array}$} & Questionnaire & $\kappa=0.05, T=0.29, p=.774$ & Questionnaire \\
Beings & RSVP-M & $\kappa=0.31, T=2.15, p=.032$ & $\kappa<0.01, T=0.00, p=1.000$ \\
$\begin{array}{l}\text { Nature of } \\
\text { Harm }\end{array}$ & Questionnaire & $\kappa=-0.18, T=-1.03, p=.301$ & \\
& RSVP-M & $\kappa=-0.07, T=-0.40, p=.692$ & $\kappa=.127, T=0.86, p=.392$ \\
$\begin{array}{l}\text { Uncontroll- } \\
\text { able }\end{array}$ & Questionnaire & $\kappa=-0.19, T=-1.21, p=.227$ & \\
& RSVP-M & $\kappa=-0.04, T=-0.30, p=.767$ & $\kappa=-0.02, T=-0.20, p=.846$ \\
\hline
\end{tabular}




\section{DISCUSSION}

The aim of this study was to investigate offence-supportive beliefs in CSOs using interview, questionnaire and experimental measures. It was hypothesised that CSOs would show evidence, according to each of these measures, of beliefs with themes matching Ward and Kennan's (1999) five ITs. In terms of interview data, this hypothesis was supported. Of the 33 CSOs interviewed, only one CSO (who strongly denied his offences) failed to utter statements that could be classed as IT-consistent. This finding aligns with a recent interview study of CSOs in which all 22 participants were found to endorse ITs (Marziano, 2002). It also accords with the many other interview studies that have shown CSOs utter cognitive distortions when being interviewed (see Gannon \& Polaschek, 2006). As with Marziano et al.'s (2006) study it was also found that the most commonly endorsed IT in the current study was Children as Sexual Beings, while the least endorsed was Entitlement.

In keeping with the hypothesis, CSOs also showed evidence of holding offencesupportive beliefs when their MOLEST responses were compared with those of offender controls. Again, this finding aligns with previous research showing that CSOs can be successfully distinguished from comparison groups using the MOLEST and other questionnaire measures of CSOs' beliefs (see Gannon \& Polaschek, 2006). When MOLEST items were grouped according to IT type CSOs provided higher endorsement levels than OCs for Children as Sexual Beings, Nature of Harm, and Uncontrollable. This is the first time that CSOs' and OCs' item responses to an existing CSO questionnaire have been grouped and analysed according to IT-themed belief clusters. This finding will be useful information for researchers wishing to develop questionnaire measures designed to tap ITs or similar schemas.

It was interesting to compare participant's MOLEST responses with their IM and SD scores. Impression management (IM) refers to a general tendency to deliberately alter 
one's responses to questionnaires in order to appear more socially desirable. Although CSOs in the current study showed higher levels of IM endorsement than OCs, no correlation was found between IM and MOLEST scores, and adjusting MOLEST scores for IM responses did not alter the pattern of results. Self-Deception (SD), which is the tendency to unconsciously and positively distort one's self-perception, was not found to differ between CSOs and OCs and, again, adjusting for the presence of SD made no difference to the pattern of MOLEST responding. These findings are similar to other studies in which socially desirable responding has not appeared to account for CSOs' responses to questionnaire measures of offence-supportive beliefs (see Gannon \& Polaschek, 2006). This could suggest that, contrary to some claims, CSOs do answer such questionnaires honestly. On the other hand, the tendency to be honest about one's flaws and foibles does not necessarily predict the honesty with which one answers items relating children and sexual themes. That is to say, CSOs and/or OCs might show a general tendency to answer questionnaires with candour, but still answer sensitive questions regarding child-related sexuality in a false manner designed to meet assumed administrator expectations. Thus, it can be very hard to deduce from CSO questionnaires exactly what phenomena respondents' answers reflect.

Our findings that CSOs showed evidence of offence-supportive beliefs on interview and questionnaire measures merely add weight to a body of research demonstrating similar findings. Of key interest, however, was whether CSOs would show evidence of such beliefs when their responses to the RSVP-M task were compared with those of controls. Against predictions, no differences could be found between the two offender groups. This result held even when three ITs were singled out and analysis of each IT included only those CSOs who had endorsed that IT, first in the interview and then on the questionnaire. Recall also that CSOs in this study had been interviewed about their offending immediately prior to completing the RSVP-M. Presumably the 
interviewing process would have activated any offence-supportive beliefs they held, meaning CSOs were well primed before taking part in the RSVP-M.

On the one hand, the RSVP-M results mirror the experimental results of Gannon et al. (2006) and those obtained in Studies One and Two in this thesis. The current result suggests the null findings in these earlier studies did not arise because CSO participants' beliefs were not primed, or because certain CSOs included in the analyses obscured group differences. On the other hand, the RSVP-M result runs contrary to the questionnaire and interview results found in this and other studies, and also to theoretical and practical work asserting that CSOs generally hold entrenched beliefs that set them apart from others.

There are two very broad interpretations that can be made in respect of this finding. One interpretation is that design flaws in the RSVP-M experiment (and the other cognitive experiments conducted in this thesis) led to a failure to statistically distinguish CSOs from controls. Certainly the current study had limitations, which will be discussed shortly. The other interpretation is that CSOs hold offence-supportive beliefs to a lesser extent than the literature and current treatment practices imply; an interpretation that will also be discussed. Before turning our attention to these interpretations, however, a final and very important result must be addressed; the lack of agreement between the IT ratings that each of the three measures assigned to CSOs. There was nonsignificant agreement between the three measures for Children as Sexual Beings, Nature of Harm, and Uncontrollable, meaning that IT classification agreements were either at or below levels that would be obtained by chance. The lack of correlation between RSVP-M ratings and those on the conventional measures is perhaps not surprising in light of the fact that cognitive experiments have typically not found evidence of CSOs' beliefs, while questionnaire and interview typically have. In other words, research thus far has suggested experimental results might not align with those generated via more traditional means. Much more surprising, however, is the lack of agreement between questionnaire and 
interview ratings of CSOs' IT-themed beliefs. While previous research has found that interview techniques reveal more distortions than questionnaires (Neidigh \& Krop, 1992) one would still expect agreement between the types of endorsements being made by CSOs on both measures. This study is apparently the first to investigate this highly important relationship. It is vital that researchers and clinicians understand differences in the nature of responses generated from questionnaires and interviews as these are the two main methods of CSO beliefs assessment. Therefore, there is a strong need for further research to find out whether the current lack of agreement between the two measures is a general finding or unique to this study.

As mentioned, there were limitations in the RSVP-M experiment that may have affected study outcomes. First, sample sizes were fairly small, which may have made it difficult to detect differences. However, the overall pattern of responding did not generally run in the expected direction-offender controls' RSVP-M scores actually tended to run in a slightly more "distorted" pattern of responding than CSOs' scoresindicating that the null RSVP-M results were unlikely to be attributable to sample size. Nevertheless, replication of this study using larger sample sizes would be useful.

The study was also limited by the lack of a non-offending control group. Offenders in general are thought to have beliefs and attitudes that set them apart from nonoffenders (Walters, 1995), so perhaps more distinct differences might emerge between CSOs' and nonoffenders' RSVP responses if non-offending samples are included in future RSVP-M research. Nonoffenders could also provide norms that would allow CSOs who show no Dangerous World or Entitlement beliefs (according to conventional measures) to be removed from analysis when looking at RSVP responses to these ITs.

The RSVP-M experiment might also have been improved through inclusion of more, or different, passages. Only four passages for each IT were presented to participants in this study and prior testing was not conducted to ensure that passages most effectively 
represented IT themes and could be related to by CSOs. The experiment could be replicated with more passages that are demonstrably likely to trigger CSOs’ ITs.

Despite potential limitations of the RSVP-M experiment, the current results are not isolated. They add weight to the two previous pieces of experimental research in which evidence for CSO-specific cognition has not yet been detected, despite the fact that in each case the experimental design was previously used in other studies to detect information processing differences between groups. If CSOs generally have abnormal beliefs why don't they appear to process information differently to comparison groups? Researchers must continue to probe this question, seeking new and improved methods for experimentally testing the way CSOs attend to and interpret their worlds. It is possible that CSOs do not typically hold entrenched beliefs that significantly differ from the beliefs of other men and that can be isolated and probed at will.

The lack of correlation between the three methodologies also indicates that different measures can introduce different response artefacts. Questionnaire and interview measures have suggested that such beliefs are common among CSOs, but without knowing exactly what phenomena it is that CSO questionnaires and interviews measure scientists must remain open-minded about this issue.

To summarise, this concluding study has used a unique, multimethod approach to investigate the offence-supportive beliefs of CSOs. All but one CSO participant showed signs of holding abnormal beliefs with IT-consistent themes when being interviewed. Similarly, CSOs appeared to show higher levels of belief in the IT-themed statements listed in a questionnaire measure. However, when an implicit experimental measure of ITconsistent beliefs was used CSOs could not be distinguished from OCs. Furthermore, there was no agreement between the three measures in terms of the types of beliefs they classified each CSO as holding. These results indicate that researchers must continue to seek new ways of conceptualising and investigating the nature of CSO cognition. 


\section{Chapter Seven}

\section{What Were They Thinking? General Conclusions}

Personally, I did not meet any [child sexual] offenders who did not hold distorted beliefs when explaining what actually happened. We are talking about sexual offences against children.

- Anonymous Reviewer ${ }^{7}$

\section{Introduction}

This thesis began by outlining the popular theory that CSOs hold offencesupportive beliefs. As the above quote illustrates, the intuitive appeal of this theory rests on the fact that it posits a common locus of causation among CSOs, and seems to explain how CSOs can commit crimes that most people find abhorrent. Unfortunately, however, the overall approach by which this theory has so far been tested has somewhat lacked in scientific rigour. Methods examining the theory have typically measured the extent to which CSOs utter or endorse "cognitive distortions", on the rationale that those distortions represent the contents of stable cognitive structures that occupy a fundamental causal role in the generation of sexual offending behaviour. In the preceding pages it has been argued that cognitive distortions (i.e., CSOs' seemingly distorted-sounding statements) can in fact represent other phenomena, such as socially desirable responding and post-hoc rationalising. The primary aim of this thesis has been to investigate CSOs' beliefs using methods less susceptible to socially desirable responding and less dependent on introspective and retrospective reporting. None of the experimental designs employed in this thesis have previously been used within the CSO population. Furthermore, Studies two and three represent the first occasions in which CSOs' beliefs have been primed prior to experimental investigation.

\footnotetext{
${ }^{7}$ This comment was written by an anonymous peer reviewer of a well-known psychology journal.
} 


\section{Lexical Decision Task}

In Chapter Four CSOs' beliefs were investigated using a robust experimental technique called the lexical decision task. Lexical decision tasks have long been used to study the contents and nature of cognitive structures. Although there are different types of lexical decision task, all draw upon the fact that people are faster to process words that follow a relevant cue (e.g., a related context sentence) than words that do not. The type of lexical decision task employed in this thesis was previously used in other populations to probe expected differences between readers' beliefs (e.g., Baldwin et al., 1993; Kay \& Jost, 2003; Mikulincer, 1998; Mikulincer et al., 2000).

CSOs, OCs, and nonoffender controls were presented with incomplete sentences that could be interpreted in an offence-supportive or non offence-supportive manner, depending on the reader's predominant beliefs. Each sentence stem was followed by a word that was either consistent or inconsistent with an offence-supportive interpretation of the preceding sentence. It was hypothesised that, compared to controls, CSOs would respond faster to words that completed sentences in a manner consistent with offencesupportive beliefs than sentences inconsistent with offence-supportive beliefs. Contrary to predictions, CSOs did not show a different overall pattern of responding to consistent and inconsistent words than either control group. When the data was grouped according to the belief themes that characterise individual ITs, CSOs remained statistically indistinguishable from controls for four out of the five ITs (Children as Sexual Beings, Dangerous World, Entitlement, and Nature of Harm). Compared to OCs, CSOs did show accelerated recognition for words supporting the Uncontrollable IT. While that result could suggest that CSOs hold Uncontrollable beliefs, this interpretation is questionable for two reasons: 1) CSOs' responses to this IT did not differ from community controls', and 2) OCs' responses to Uncontrollable were unusually elevated. 
Overall, results from the lexical decision task indicated that CSO participants did not interpret sentences in line with offence-supportive beliefs. This might indicate that CSOs do not hold offence-supportive beliefs to the extent currently posited in the literature. Or, as will be discussed, it could suggest that current conceptions of the structure of such beliefs are inadequate. What is certain-as discussed in Chapters Four, Five and Six - is that the results highlight the need to continue to use cognitive experimental methods to study CSOs' beliefs.

\section{Primed Memory Study}

Chapter Five presented a study in which a different experimental method was used to investigate beliefs relating to the Children as Sexual Beings IT. In order to maximise the chance that these types of beliefs were activated, and to study the effects of activation, visual primes (i.e., pictures of scantily-clad children) were presented to half the CSO and OC participants. Under the guise of an age-judging task, CSOs and OCs were exposed to pictures of either semi-clothed children (priming condition) or clothed, mature adults (control condition). Participants then read ambiguous sentences describing children's actions that could be interpreted in a sexualised manner. To disguise task aims and ensure that more general interpretation or response biases did not exist between groups, neutral sentences and ambiguous sentences that could be interpreted in a threat-consistent manner were also presented. Next, participants completed a surprise recognition test in which half the potentially sexual sentences were re-presented in an unambiguously sexual form, and half in an unambiguously nonsexual form. Reworked neutral sentences and unambiguous threat-related sentences were also presented.

As expected, CSOs in the priming condition looked at pictures of children longer than OCs. Because viewing times are an indicator of sexual interest (e.g., Abel et al., 1998; Gress, 2005), this suggested that beliefs which might lead CSOs to make sexualised interpretations of children's behaviour should be primed. Also as expected, none of the 
four groups (i.e., primed and unprimed CSOs and OCs) differed in their responses to neutral and threat-related recognition sentences, indicating that results were not influenced by more general memory biases. Contrary to hypotheses, primed and/or control CSOs did not show a recognition bias for sexualised sentences, nor faster response times to these sentences. This finding suggests that CSOs did not interpret the original sentences in line with offence-supportive beliefs, which is surprising given that the Children as Sexual Beings IT is meant to be widely held by CSOs (Marziano et al., 2006) and is thought to be CSO-specific (Ward et al., 2007). As with the first study in this thesis, the results raise questions about whether CSO participants actually held offence-supportive beliefs, or whether current conceptualisations of such beliefs are accurate. The findings also imply that the null findings of the lexical decision task did not occur because CSOs' cognitive structures weren't appropriately activated. Once again, this study highlights the need for further experimental research within the sexual offending field.

\section{The Multimethod Study}

In the concluding study to this thesis, data regarding the content of CSOs' beliefs was gathered using three methods: interview, questionnaire, and cognitive experimental. Results generated by these methods were then triangulated to see whether the methods showed agreement regarding the extent and nature of offence-supportive beliefs. CSOs were interviewed and the content of each CSO's statement was categorised according to the five IT types. Next, with CSOs' beliefs presumably primed by their interview discussions, CSOs and OCs participated in an experimental task (Rapid Serial Visual Presentation-Modified), which uses sentence reading times to explore the content held in cognitive structures. RSVP-M tasks are based on the finding that readers process sentences faster if the sentences continue preceding text in line with the reader's mental model (Albrecht \& O'Brien, 1993). This finding has successfully been used to investigate differences in the content of cognitive structures that particular groups of readers have 
used to comprehend texts (Macleod \& Cohen, 1993; Wingrove \& Bond, 2005). Finally, CSOs and OCs completed a questionnaire measure (MOLEST; Bumby, 1984), which is often used to assess CSOs' beliefs for treatment and research purposes.

As hypothesised, CSOs showed evidence of holding distorted beliefs according to interview data: 32 out of 33 CSOs made statements that appeared to endorse beliefs pertaining to one or more of the five ITs. CSOs also appeared distorted according to the questionnaire measure, although they disagreed on average with MOLEST items, their ratings were significantly higher than OCs' average item ratings. Against predictions, however, CSOs did not show evidence of holding distorted beliefs on the RSVP-M task; their pattern of responding to IT-consistent and IT-inconsistent sentences could not be distinguished from that of OCs. This finding held true for each of the five ITs. Even when RSVP-M responses to three ITs (Children as Sexual Beings, Nature of Harm, and Uncontrollable) were reanalysed with inclusion of only those CSOs who demonstrated IT endorsement on the conventional measures, CSOs could still not be statistically discriminated from controls. Thus, once again, the experimental result obtained in this study seemed to suggest that CSO participants did not hold distorted beliefs that produce offence-supportive interpretations of social stimuli.

An important question in this study was whether the three methods would concur in terms of the types of IT that each CSO was deemed to hold. To investigate, CSOs were categorised as either endorsing or not endorsing Children as Sexual Beings, Nature of Harm, and Uncontrollable according to each of the measures. Categorisations made via the RSVP-M did not show agreement with either the interview or questionnaire categorisations, and considerably fewer CSOs were categorised as endorsing each IT according to the RSVP-M method. This result did not come as a great surprise, given the fact that experimental techniques so far have not detected evidence of widespread offencesupportive beliefs among CSOs. More surprising, however, was the finding that although 
the interview and questionnaire techniques rated similar numbers of CSOs as endorsing each IT, the two methods showed considerable variation regarding the types of belief each CSO was deemed to hold. Overall, the findings from this third study raise important questions about the phenomena and potential artefacts measured by interview, questionnaire, and experimental methods.

\section{Limitations}

Across the three studies, findings seemed to suggest that the CSOs tested did not hold beliefs whose contents map onto the ITs identified by Ward and Keenan (1999). Of course, an alternative explanation is that CSO participants did hold distorted beliefs, but methodological flaws in all three studies prevented the detection of those beliefs. Although all methods in this thesis were based on methods that have been successfully deployed to find expected group differences within other fields, like any experimental research the present studies had limitations. For instance, this thesis investigated a sample of child sexual offenders that by its very nature was biased. All CSOs who took part in the three studies volunteered to do so. Perhaps CSOs were more prepared to take part because they had adjusted their thinking in relation to sexual offending, and thus were not as defensive as CSOs who shunned the research. Of course, forcing unwilling individuals to participate in psychological research is hardly a viable option, so this idea must remain a speculative one. Another potential sampling bias is the fact that only CSOs who had actually been detected and incarcerated for their crimes were studied. There may well be key differences between these men and those who abuse children but avoid incarceration. Qualities characteristic of incarcerated men might have existed prior to imprisonment (e.g., less expertise grooming and silencing victims, greater desire for detection by others), or might be a consequence of imprisonment (e.g., greater reflection on offending, willingness to change). These factors should be considered when using the current findings to draw conclusions about CSOs as a whole. Also worth considering is the fact 
that out of the 67 men who took part in one or more studies in this thesis, seven had previously received cognitive-behavioural treatment for their offending. Although all seven had since re-offended, the previous attempts at belief modification may have altered their responses somewhat.

The requirement that participants be reasonably good at reading also created a sampling bias. Well-read offenders are not necessarily representative of the offender population at large. Perhaps CSOs who are poor readers (and therefore excluded from this type of research) are for some reason more likely to hold offence-supportive beliefs than good readers. For instance, poor readers may have cognitive limitations that make them less likely to analyse and modify their belief systems while in prison. While the idea that excluding poor readers may have skewed results is worth bearing in mind when considering current findings, in the absence of neuropsychological testing this idea is mere conjecture.

The requirement for good readers also resulted in fairly small sample sizes being used in each study. This could have made it difficult for group differences to reach statistical significance. Having said that, overall patterns of responding in each of the studies did not move in the expected direction. That is, CSOs' responses did not tend to look more distorted than controls. Therefore, even if group sizes were increased, it seems highly unlikely that significant differences in the expected direction would have been found between CSOs and controls.

Finally, it should be noted that the settings in which this research took place were often less than ideal. The makeshift experimental facilities that were set up in different units across different prisons sometimes left participants exposed to noise interference and interruptions from others while completing reaction time tasks. Compounding this was the fact that not all participants seemed to maintain concentration well and some even talked during tasks. In reaction time studies cognitive interference and lapses in attention can 
skew overall results. Although attempts were made to statistically compensate for this (i.e., all reaction time data was transformed through Windsorising), it would have been preferable to work in experimental settings that minimised distractions and encouraged participants to remain focused on the task.

Regardless of potential limitations, the overall findings in this thesis raise some substantial questions regarding current conceptions of CSOs' beliefs. In the following section some of these questions will be addressed. First it will be asked whether offencesupportive beliefs are as widespread as commonly thought and, if not, what implications this has for the underlying nature of cognitive distortions. Second, traditional experimental approaches towards CSOs' beliefs will be examined in the light of recent conceptions about the dynamic and embodied nature of cognitive processes. The discussion will then turn to research and treatment implications that arise from the findings generated within this thesis.

\section{Current Conceptions of CSOs' Beliefs}

In the introduction to this thesis it was argued that researchers and clinicians tend to accept the idea that most or all CSOs hold distorted beliefs. Consequently, a key aim of current treatment programmes is to change or restructure these beliefs. However, the findings that emerged from the three studies in this thesis suggest that there may be something wrong with current conceptions of CSOs' beliefs. This raises two key possibilities: either offence-supportive beliefs do not play as predominant a role in CSOs' crimes as is currently thought, or offence-supportive beliefs are major aetiological factors but revisions are needed in the way that they are conceived of and studied. Each of these possibilities will now be considered in turn.

\section{Prevalence of CSOs' Offence-Supportive Beliefs}

Offence-supportive beliefs or schemas are generally thought to be widespread among CSOs. These cognitive structures are also thought to differentiate CSOs from other 
individuals. Yet evidence of IT-themed beliefs was not uncovered in this thesis. This is not the first time evidence of CSOs' offence-supportive beliefs has failed to materialise under experimental investigation; a null result also emerged when Gannon et al. (2006) used an implicit memory study to investigate beliefs clustered within four IT categories. Recall that Gannon et al. used a similar paradigm to that used by Stermac and Segal (1989), inasmuch as CSOs and controls were compared on how they interpreted vignettes describing adult-child sexual contact. However, rather than asking participants to selfreport on their interpretations, Gannon et al. adopted an implicit, recall-based approach designed to minimise experimenter effects and socially desirable responding. The null result that emerged prompted Gannon et al. (2006) to suggest that there is some doubt surrounding “...existing cognitive distortion literature predicting that child molesters are generally guided by a number of implicit theories" (p. 14). Results from the current three studies could be interpreted in a similar way. Of course, the findings of this thesis are not being used to deny the importance of beliefs as aetiological factors; there are undoubtedly CSOs whose beliefs are abnormal and supportive of child sexual abuse. However, it is possible that these beliefs are less widespread among CSOs than is currently thought, or that they are not especially specific to this population of men. In short, their role in the commissioning of child sexual offending may have been overstated.

The strong sense in the literature that CSOs typically hold abnormal beliefs is predominantly based on the fact that CSOs so often seem to utter or endorse seemingly distorted-sounding statements (i.e., cognitive distortions). However, it is far from obvious that such utterances or endorsements are reliable indicators of distorted beliefs. They might in fact reflect a host of other phenomena, ranging from attempts to appear more agreeable (to oneself or others) or to meet presumed experimenter expectations, through to attempts to create rational accounts of seemingly irrational behaviour or to simply provide fairly accurate event descriptions that are mistakenly classed as distorted. The fact that in 
the third study of this thesis CSOs endorsed cognitive distortions according to interview and questionnaire measures but did not show evidence of abnormal beliefs on the RSVPM may indicate that conventional, self-report methods measure phenomena other than beliefs. Like other experimental techniques used in this thesis, the RSVP-M task was designed to minimise the opportunity to provide false responses and the need to introspect on implicit cognitions. It is intriguing that under such conditions CSOs who had seemed distorted using conventional measures appeared no different to offender controls. In addition, the types of beliefs that CSOs were deemed to hold differed according to which cognitive distortion measure was used (i.e., interview or questionnaire). If cognitive distortions tend to represent beliefs, why didn't CSOs' endorse the same sorts of themes across the two methods?

Given the somewhat questionable evidence supporting the theory that CSOs hold distorted beliefs, it is surprising that the theory is so often propounded, implicitly or explicitly, in the literature. It seems there is something about the abhorrent and deviant nature of CSOs' crimes that invites examination of the perpetrators' beliefs. This idea is illustrated by the words quoted at the start of this discussion, which were made in defence of the idea that CSOs as a group hold distorted beliefs: "We are talking about sexual offences against children." While psychologists typically approach human behaviour with the underlying question, "Why did they?", when the topic of child sexual abuse is raised the question seems more to become, "How could they?" So why do current attempts to understand CSOs' actions seem to focus rather strongly on their cognitive structures?

Maruna and Mann (2006) have pointed out that researchers might be inclined to target stable cognitive drivers when explaining CSOs' actions because it is a relatively comfortable avenue to pursue. It is reassuring to think that beliefs endorsing the undesirability of child sexual abuse have a strong preventative effect. The alternative view - that in the presence of strong environmental, emotional, and biological cues such 
beliefs may be overridden—is threatening, as it implies that even "good" people can commit sexual crimes. Maruna and Mann suggest that those involved in the criminal justice system might feel particularly unsettled by the idea that bad circumstances, rather than bad people, can drive criminal actions. As the authors point out,

"After all, excuses can undermine the very foundations of criminal justice. If we are to punish (or arrest, convict, study, classify, etc.) a person as an offender, the individual needs at some level to be responsible for the crime." (p. 12).

In the upcoming 'Treatment Implications' section of this discussion the issue of offender responsibility and blame will be elaborated upon.

Perhaps another reason for isolating CSOs' beliefs as a general cause of child sexual offending is to simplify treatment. For one thing, in forensic settings CSOs are typically not engaged in treatment till they near the end of their sentence, meaning that years have usually passed since they last offended. Therefore, when looking to modify current cognitions that contributed to a CSO's offending years ago, the therapist targets cognitions that have remained relatively stable over time. The fact that CSOs are treated in restrictive forensic settings also renders the targeting of stable beliefs easier than targeting temporary appraisals. Unlike non-offending clients, CSOs cannot go out into their everyday worlds between therapy sessions and record, as they arise, the transitory thinking errors that occur in response to environmental input. Also, within the confines of the forensic setting therapists cannot readily help CSOs deal with the environmental stressors that contributed to earlier offending. These factors all serve to make a systems-based model of therapeutic change difficult and thus increase the attractiveness of a model in which stable beliefs or schemas form the therapeutic focus. As for the popular idea that CSOs' distorted beliefs are widespread; because treatment of CSOs usually takes place in group formats it is probably easier to assume that all group members need belief 
modification than to try to single out those who do and those who do not. Nevertheless, the current thesis is not the first work to argue that treatment programmes should tailor therapy to better suit the psychological needs of individual clients (e.g., Hanson et al., 2002; Kirsch \& Becker, 2006; Marques, Wiederanders, Day, Nelson, \& van Ommeren, 2005 ).

Regardless of any intuitive or practical leanings they may have towards beliefbased explanations of child sexual offending, it is important for social scientists to bear in mind that highly undesirable behaviours can be understood without reference to perpetrators' beliefs or schemas. For instance, one of the founding, and perhaps most wellknown, social psychology experiments was that conducted by Stanley Milgram (1963). As the reader is no doubt aware, Milgram found that in certain social circumstances seemingly ordinary individuals can be induced to torture experimental confederates with dangerous (and even lethal) doses of electricity as punishment for failing to remember word pairs. In their attempts to explain why Milgram's participants so behaved, psychologists have not declared that those participants probably held beliefs supporting the electrocution of people with less-than-perfect memories. Rather, explanations have typically focused on the interaction between general human psychological characteristics and the unusual demands of the experimental setting.

This sort of approach recognises that it is possible to elicit abhorrent behaviour in the absence of distorted or abnormal beliefs. In fact, the concept of cognitive deconstruction introduced at the start of this thesis suggests that sexual offending can occur when inhibitory, non-distorted beliefs are actually overridden. According to the cognitive deconstruction literature, humans can perform undesirable acts when factors such as strong sexual arousal and intense emotion leave them highly motivated to pursue a particular goal. In this situation, beliefs are swept aside, leaving the individual unhampered by the usual concerns that inhibit terrible misdeeds. Thus, CSOs may differ 
from others more in terms of the nature and strength of their emotional, biological, and environmental inputs than in terms of their beliefs.

To summarise, the results of this thesis may indicate that CSOs do not hold abnormal, offence-supportive beliefs to the extent portrayed within the current literature and implied by current treatment practices. While interview and questionnaire techniques have often revealed evidence of CSOs' cognitive distortions, this should not be equated with evidence of abnormal beliefs (at least not until the mechanisms driving cognitive distortions are better understood). Although it might be challenging to rethink the idea that CSOs typically hold offence-supportive beliefs, it is also vitally important that social scientists remain open-minded to alternative hypotheses and rigorously test the models under which they work.

\section{The Nature of Offence-Supportive Beliefs}

It has been discussed in this thesis that certain assumptions underlie the use of interviews and questionnaires in the study of CSOs' beliefs. In preceding pages particular attention was paid to the assumption that beliefs can be quantified by measuring CSO's seemingly distorted-sounding statements. There are other assumptions about the nature of offence-supportive beliefs implied by interview and questionnaire methods. First, the way that such methods are applied indicates an assumption that beliefs are propositional in nature. Also, they assume that beliefs are static entities that can be picked out and studied in isolation from other psychological, biological and environmental inputs.

To illustrate, the questionnaires used to assess CSOs are essentially lists of propositional statements (e.g., "Sometimes, victims initiate sexual activity", "I believe that sex with children can make the child feel closer to adults"). Within environments that are far removed from their everyday lives, CSOs rate the extent to which they believe these propositions, or "cognitive distortions", are true. In other words, it is assumed that beliefs are propositional and static "things" that are readily available to be measured. Similarly, 
interview assessments often aim to uncover beliefs by having CSOs speak to relative strangers-usually in correctional settings-about offending that happened some time ago. Propositions that seem to minimise, justify, excuse, or rationalise that offending are taken as evidence that the speaker has a distorted way of interpreting his social world which has long-term behavioural consequences. CSO treatment programmes typically place strong emphasis on changing these so-called cognitive distortions. For instance, Marshall and Barbaree (1999) describe how CSOs in treatment are asked to describe their offences to a therapy group so that members can challenge statements that seem to minimise or excuse the speaker's role in his offences. In addition, the success of treatment programmes is in part monitored by the shift that CSOs show on questionnaire lists of distorted propositions. "Change the propositions," these treatment approaches seem to say, "and you will produce stable changes in the way the offender interacts with his world". This view flies in the face of advances in cognitive science wherein cognitions such as attitudes and beliefs have come to be seen as non-propositional in nature (e.g., Churchland, 1992; Rumelhart \& McClelland, 1986; Wright \& Schneider, 1999).

The first study in this thesis worked under similar assumptions to those currently operating within the CSO literature. That is to say, CSOs and controls were asked to respond to offence-related propositions in a setting that contained few, if any, relevant primes. This might explain why no differences emerged between groups. In the second two studies attempts were made to overcome these conceptual issues. The second and third studies investigated the way CSOs process non-propositional information following exposure to offence-relevant primes. However, these two approaches may also have failed to find evidence of offence-supportive beliefs because CSO participants were still too removed from real-life offending situations.

Increasingly, cognitive science is moving away from a view of mind as an abstract information-processor whose links to the world have little theoretical or causal importance 
(see Clark, 1997; Damasio, 1994; Pecher \& Zwaan, 2005; Wilson, 2002). Beliefs are not seen as static representations of a world that sits outside the brain, but instead as fluid patterns of neuronal activity that unfold in conjunction with emotional, biological, and environmental inputs (Clancey, 1993). Thus cognition is seen as situated (i.e., enmeshed, on a moment-by-moment basis, with the environment) and embodied (i.e., fused with bodily perceptions, sensations, and movements).

Such an approach emphasises the synchronous nature of the interaction between beliefs and other inputs. Beliefs produce adjustments in behaviour that bring about changes in the individual's environment (and his placement in it), which in turn bring about changes in the stimuli being received, which in turn impact upon the individual biologically and alter his patterns of neuronal activity. In this way, a constant feedback loop exists between the individual's cognitions, his behaviour, his biology, and his environment.

Although the experiments in this thesis tended to look more at the way that CSOs' process information than the propositions they endorse, and although attempts were made to prime CSOs' beliefs, all three studies nevertheless treated beliefs as stable representations of the world "out there", rather than patterns of processing that unfold in conjunction with other dynamic factors. Perhaps this fact could account for the null results across the three studies.

The concepts of embodied and distributed (or situated) cognition have recently been introduced to the sexual offending field via a ground-breaking article by Ward and Nee (in press). In their article Ward and Nee use recent philosophical and cognitive science concepts to critique a view of CSOs as rational, disembodied decision-makers. The model upon which Ward and Nee focus their argument is called the Reasoning and Rehabilitation programme (Ross \& Fabiano; cited in Ward \& Nee, in press), a programme that essentially aims to reduce reoffending by teaching offenders how to reason about, and 
plan, their actions. Ward and Nee argue that such programmes fail to take into account the role that emotional, environmental, and experiential inputs play in decision-making processes. In discussing emotional influences, Ward and Nee reiterate the point which was covered in the introduction to this thesis that humans typically make decisions based on intuitive, emotionally-driven processes; it is only in the aftermath of these decisions that rational judgement is invoked as a guiding principle. Ward and Nee also highlight the fact that humans are cognitively and behaviourally malleable creatures who both shape, and are shaped by, their environments. Thus, to quote Ward and Nee, “...it is a mistake to view human nature as biologically fixed and contained inside people's heads" (p. x). It is hoped that researchers will follow Ward and Nee's lead and strive to stay abreast with current conceptions of the nature of cognition when conducting studies of it.

Of course, it is not being argued that researchers cannot understand CSOs' beliefs without conducting exhaustive (and impossibly exhausting) analyses of CSOs' environments. As Wilson (2002), points out, the fact that environment plays a causal role in cognition does not mean that researchers and theoreticians have to look at the entire system surrounding cognition. As an analogy, although the human heart is part of a complex biological system, scientists have learnt much about it by studying it in isolation from living bodies. What is being argued here is that researchers should always be very mindful of the types of inputs and interactions that might influence the way in which beliefs operate.

Taking a less symbolic, more distributed view of cognition might help to explain the implicit association test (IAT) results discussed in the introduction. Recall that a number of researchers have used IAT experiments to investigate links between particular concepts among CSOs versus controls. It has been found that CSOs have stronger semantic links between the concepts of children and sex (Gray et al., 2005; Mihailides et al., 2004; Nunes, 2007), and the concepts of sex and uncontrollability and sex and 
entitlement (Mihailides et al., 2004). As argued, these findings do not necessarily mean that CSOs hold mental representations that, for instance, portray children as consenting sexual agents. However, the findings do seem to show that patterns of neural activity that occur when CSOs receive inputs related to sexual experiences tend to be associated with input experientially related to children. A question that needs to be resolved is whether or not these associated patterns of activity exist prior to (and play a causal role in) sexual offending, or whether they occur in response to sexual offending and its consequences.

\section{Research Implications}

Perhaps the most important research implication to arise from this thesis is the clear need to continue to apply cognitive-experimental techniques in the study of CSO cognition. Questions raised in this thesis about the way that offence-supportive cognitions are arranged, and operate, in CSOs simply cannot be resolved by asked CSOs to report on their own thoughts. While there is undoubtedly a place for interview- and questionnairebased research in this field of enquiry, there must be recognition that that use of self-report techniques can only take social scientists so far. As long as the focus falls solely on cognitive distortions as indicators of the complex cognitive systems utilised by CSOs, social scientists will struggle to understand how these systems actually function when in action. Thus, there is a strong need for continued use of techniques that are designed to implicitly capture offence-relevant cognition as it unfolds. Currently the implementation of these implicit techniques within the field of sexual offending is in its infancy, leaving the way open for researchers to refine techniques and develop more sophisticated ways of applying them.

For instance, there is a need for studies to explore in greater depth the effects of alternative primes on information processing in CSOs. Although attempts were made to prime CSOs' beliefs in the final two studies of this thesis, these studies represent metaphorical "first steps" for research into the effects of priming on CSOs' information 
processing. There are many primes that could be used to try to activate CSOs' beliefs (e.g., emotional states, sexual arousal, moods, alcohol consumption) and many ways in which these primes could be manipulated and applied. Sexual offenders appear to be highly impulsive (Kirsch \& Becker, 2006), implying that situational primes may have particular importance in the study of CSOs. It is hoped that through the creative application of priming variables to the measurement of beliefs, researchers will gain a better understanding of the nature and extent of CSOs' beliefs.

This thesis also indicates that there is a strong need to develop experimental stimuli that more accurately imitate situations likely to be experienced by CSOs in their day-to-day lives. For instance, rather than having offenders respond to written stimuli describing offence-relevant situations they could watch realistic videos while being prompted to make automatic, keypad-based responses to, or recall particular aspects of, the scenarios being presented. Computers could also be used to allow feedback between the offender participant and his environment. For instance, if participants are required to make a series of decisions or responses in reaction to events unfolding on a computer screen, and each decision has implications for the way that events unfold, a deeper understanding may be gained about the role of cognition in the offending process. Virtual technology could also be used such that participants interact with virtual children while measures are taken of the way in which CSOs attend to stimuli, the interpretations they make, and the factors that influence their responses. Using virtual technology, participants could even be embedded in simulated environments and scenarios that are offence-related and their interactions with these environments could be assessed. The themes represented by Ward and Keenan's ITs may be used to inform the type of experimental stimuli presented within realistic experimental designs. For although evidence for ITs as cognitive structures housing belief-based knowledge has not been found in this thesis, the themes captured by these ITs are still likely to have value (as evidenced by results from IAT 
studies discussed earlier). If cognition is best conceived of as being distributed across environments, when designing realistic experimental settings those settings could be designed to encourage perceptions of children as sexual beings, for example, or of the world being a dangerous place.

Research into alternative drivers of cognitive distortions (i.e., CSO's distortedsounding statements) would also be a valuable pursuit. Measuring cognitive distortions under different personal and interpersonal situations may reveal processes-both internal and external to the offender - that influence the generation of distorted-sounding propositions among CSOs. Research of this nature may enable psychologists to create situations in which the belief components of cognitive distortions are more readily isolated.

A final research implication that must be mentioned is the need for further study into the degree of agreement between information about CSOs' beliefs that is gleaned from interview, questionnaire, and experimental methods. In the final study of this thesis these three methods generated quite different results concerning which CSOs held which types of beliefs. Even interviews and questionnaires, which are two very widely used methods of CSO belief assessment, showed no alignment in the type of information generated about CSOs. It seems rather startling that in the two decades of interview and questionnaire use within this field these methods have not been compared in terms of the information they reveal about offence-supportive beliefs. If use of these methods is to continue there is clearly a need to investigate this relationship further. By replicating (and adjusting) the multimethod approach employed in study three researchers will be able to discover whether its findings were idiosyncratic or commonplace.

\section{Treatment Implications}

The findings of this thesis have implications for the way in which CSOs are rehabilitated; if faulty beliefs are not universally held by CSOs it follows that 
rehabilitation programs may need to tailor treatment approaches that are better suited to CSOs for whom faulty beliefs play little or no role in their offending. As Kirsch and Becker (2006) have pointed out, sexual offender rehabilitation is typically group-based, with all participants receiving the same cognitive interventions. In a recent review of studies specifically assessing the effectiveness of cognitive-behavioural sexual offender treatment, Kirsch and Becker (2006) concluded that between $10 \%$ and $30 \%$ of treated sexual offenders recidivate within five years of their release. These figures certainly leave room for improvement. The authors suggested that one way of improving cognitivebehavioural treatment could be to target only those factors relevant to intervention. In light of the current findings, this may mean reducing the time devoted to restructuring some CSOs' distorted cognitions, and increasing the time spent identifying other causative factors. Many of these potential alternative drivers (e.g., emotional dysregulation, alcohol abuse, antisocial peers, environmental stressors, limited coping skills, and social skills deficits) were located within the multifactor theories of sexual offending outlined in the introduction to this thesis. Note that it is not being claimed here that these factors are currently ignored within current treatment programmes; the point is simply that, for some CSOs, the time and resources allocated to modifying beliefs could be reduced, while time spent addressing other factors could be increased.

Another implication of this thesis is that therapists need to take great care when deciding that a CSO's distorted-sounding utterance in therapy is evidence of a distorted belief. At present there is a view that cognitive distortions spoken by CSOs in therapy need to be challenged; that way a shift in thinking will be produced and the likelihood of reoffending reduced. However, if cognitive distortions are sometimes excuses being offered by the speaker to salvage his self- or public image, it is not necessarily helpful to challenge it. 
Some authors have emphasised the importance of confronting CSOs' excuses and requiring them to take responsibility for their actions (for a review see Maruna \& Mann, 2006). However, this approach goes against best practice guidelines and may be disruptive to the therapeutic relationship (Beech \& Mann, 2002; Maruna \& Mann, 2006). It also assumes that changing people's beliefs about, or attitudes towards, laws and norms will change their behaviour. Yet as Palmer (2003) has pointed out, lawful or moral behaviour is not necessarily determined by lawful or moral attitudes, for situational factors such as emotional drives and social variables can limit correspondence between attitudes and behaviour.

In addition, excuses may be associated with positive mental health because they serve to protect self esteem (Bowens, 2004; Gudjonsson, 1984; Lord \& Willmot, 2007; Maruna \& Mann, 2006). Given that low self-esteem is thought to contribute to child sexual offending (Marshall et al., 1999a) and that attacks to self esteem probably increase desire to self deceive (Thagard, 2006), challenging CSOs' excuses might actually undermine treatment aims. In support of this, some evidence has suggested that recidivism is actually negatively associated with the act of excusing or minimising one's misdeeds (see Maruna \& Mann, 2006).

Despite this, the claim that CSOs' excuses could be best left unchallenged may seem alarming to some. This concern might stem from the idea that often repeated excuses may become entrenched beliefs that facilitate future offending. In response to this concern the point must be raised that if CSOs can be helped to alter the underlying mechanisms causing them to want to believe excuses, repetition of those excuses may automatically cease. To illustrate, rather than challenging CSOs who utter excuses, it may be more helpful to teach them alternative ways to cope with feelings of distress or self-dislike. Research has shown that CSOs are more prone than other types of offenders and nonoffenders to utilise emotionally focused strategies when dealing with unwanted situations 
(Feelgood et al., 2005; Marshall et al., 1999a). In other words, CSOs focus their attention on strategies that try to deal with the emotional consequences of experiencing stressors, rather than using problem-solving strategies aimed at the stressors themselves. Thus, if CSOs are distressed by thinking about the implications of their offending, they may be more likely to generate soothing excuses rather than tackling the issue of the offending itself. By helping CSOs learn a more problem-solving approach the therapist may arm CSOs with skills that automatically reduce excuse generation, and thereby bypass the need to argue against those excuses.

One example of a treatment that obviates the need to directly challenge offencesupportive beliefs has been extended by Ward and Marshall (2007). Ward and Marshall have suggested using narrative therapy to address underlying drivers of sexual offending behaviour. The authors encourage therapists to help sexual offenders construct more adaptive self-identities that take into account offenders' needs and values and help them move towards a future in which these needs can be met in socially acceptable ways. The emphasis is on providing offenders with positive means for meeting personal goals and shaping new lives, rather than working on strategies for avoiding unwanted outcomes. Because this future-focussed therapeutic approach is not aimed at attacking self-protective belief systems or assigning blame, it seems likely that it will release CSOs from historical cycles of recrimination and consequent defensiveness. In this way CSOs who receive narrative therapy have little need to "repackage" their past crimes as acceptable acts, which should in turn prevent their excuses, justifications, and minimisations from feeding long-term offence-supportive beliefs.

Another possible explanation for cognitive distortions is that they are merely descriptions of events that actually happened. That is, sometimes CSOs might report on accurately remembered events, but their reports are interpreted as attempts to distort the facts (Maruna \& Mann, 2006). For instance, a CSO who says their victim did something 
provocative is not necessarily distorting facts, as it is not unheard of for children to explore sexual matters. Another example is that a CSO who states that he only offended while drunk is not necessarily trying to excuse his actions.

The idea that therapists need to take care not to read too much into CSOs' explanations is somewhat supported by a study conducted by Drapeau, Korner, Granger, and Brunet (2005). Drapeau et al. asked CSOs to talk about the strengths and weaknesses of an inmate treatment program. The authors found a common complaint to be that therapists sometimes disbelieved truthful descriptions of offence-related events. As one participant stated, it was "hard to talk 'cause when you do, they say you're distorting, and when you don't, you're avoiding" (p.108). If therapists are sometimes labelling accurate recollections as distorted beliefs there is a strong risk of damage to client-therapist rapport. Furthermore, by downplaying the contributory effects of alternative mechanisms that are mentioned by CSOs, therapists may be discouraged from addressing key situational factors that play a unique role in their clients' offence chains.

\section{Final Summary}

The primary aim of this thesis was to investigate a hypothesis popular in the sexual offending literature that CSOs hold offence-facilitating beliefs. In a series of novel experiments CSOs' beliefs were studied using techniques designed to side-step issues associated with self-report methods. This controlled, empirical approach yielded no evidence that CSOs hold offence-supportive beliefs, even when attempts were made to prime those beliefs. Furthermore, a multimethod analysis failed to find agreement between conventional, self-report methods regarding the belief types CSOs were found to endorse. The key conclusion to be drawn from this thesis is that research has thus far failed to produce convincing evidence that offence-supportive beliefs are widely prevalent among CSOs. This conclusion might indicate that CSOs do not generally hold such beliefs. Alternatively, it may indicate that the ways in which those beliefs are currently 
conceptualised and researched are not fully adequate. In the face of these uncertainties one point is clear: researchers must strive to rigorously and innovatively test competing theories about CSO's beliefs if they are to develop more effective treatments in this area. 


\section{References}

Abel, G. G., Becker, J. V., \& Cunningham-Rathner, J. (1984). Complications, consent and cognitions in sex between children and adults. International Journal of Law and Psychiatry, 7,189-103.

Abel, G. G., Becker, J. V., Cunningham-Rathner, J., Rouleau, J., Kaplan, M., \& Reich, J. (1984). Treatment manual: The treatment of child molesters. Tuscaloosa, AL: Emory University Clinic.

Abel, G. G., Gore, D. K., Holland, C. L., Camp, N., Becker, J. V., \& Rathner, J. (1989). The measurement of the cognitive distortions of child molesters. Annals of Sex Research, 2, 135-153.

Abel, G. G., Huffman, J., Warberg, B. \& Holland, C. L. (1998). Visual reaction time and plethysmography as measures of sexual interest in child molesters. Sexual Abuse: A Journal of Research and Treatment, 10, 81-95.

Abel, G. G., Jordan, A., Rouleau, J. L., Emerick, R., Barboza-Whitehead, S., \& Osborn, C. (2004). Use of visual reaction time to assess male adolescents who molest children. Sexual Abuse: A Journal of Research and Treatment, 16, 255-265.

Albrecht, J. E., \& O’Brien, E. J. (1993). Updating a mental model: Maintaining both local and global coherence. Journal of Experimental Psychology: Learning, Memory, and Cognition, 19, 1061-1070.

Arkowitz, S., \& Vess, J. (2003). An evaluation of the Bumby RAPE and MOLEST scales as measures of cognitive distortions with civilly committed sexual offenders. Sexual Abuse: A Journal of Research and Treatment, 15, 237-249.

Asendorpf, J. B., Banse, R., \& Mücke, D. (2002). Double dissociation between implicit and explicit personality self-concept: The case of shy behavior. Journal of Personality and Social Psychology, 83, 380-393. 
Augoustinos, M., \& Walker, I. (1995). Social cognition: An integrated introduction. Thousand Oaks, US: Sage.

Baldwin, M. W., Carrell, S. E., \& Lopez, D. F. (1990). Priming relationship schemas: My advisor and the Pope are watching me from the back of my mind. Journal of Experimental Social Psychology, 26, 435-454.

Baldwin, M. W., Fehr, B., Keedian, E., Seidel, M., \& Thomson, D. W. (1993). An exploration of the relational schemas underlying attachment styles: Self-report and lexical decision approaches. Personality and Social Psychology Bulletin, 19, 746753

Bargh, J. A., \& Chartrand, T. L. (1999). The unbearable automaticity of being. American Psychologist, 54, 462-479.

Bargh, J. A., Lombardi, W. J., \& Higgins, E. T. (1988). Automaticity of chronically accessible constructs in person * situation effects on person perception: It's just a matter of time. Journal of Personality and Social Psychology, 55, 599-605.

Barnett, V. \& Lewis, T. (1978). Outliers in statistical data. New York: Wiley.

Bartlett, F. C. (1932). Remembering: A study in experimental and social psychology. London: Cambridge University Press.

Baumeister, R. F. (1989). Masochism and the self. Hillsdale, NJ: Lawrence Erlbaum.

Baumeister, R. F. (1990). Suicide as escape from self. Psychological Review, 97, 90-113.

Baumeister, R. F., \& Wotman, S. R. (1992). Breaking hearts: The two sides of unrequited love. New York, US: Guilford Press.

Beck, A. T. (1963). Thinking and depression: Idiosyncratic content and cognitive distortions. Archives of General Psychiatry, 9, 324-333.

Beck, A. T. (1967). Depression: Clinical, experimental and theoretical aspects. NY: Hoeber. 
Beck, A. T. (1996). Beyond belief: A theory of modes, personality, and psychopathology. In P. M. Salkovskis (Ed.), Frontiers of cognitive therapy (pp. 1-25). New York: Guilford Press.

Beck, A. T., \& Clark, D. A. (1988). Anxiety and depression: An information processing perspective. Anxiety Research, 1, 23-36.

Beck, A. (1999). Prisoners of hate. The cognitive basis of anger, hostility, and violence. New York: HarperCollins.

Becker, J. V., \& Murphy, W. D. (1998). What we know and do not know about assessing and treating sex offenders. Psychology, Public Policy, and Law, 4, 116-137.

Beech, A. R., \& Fisher, D. (2002). The rehabilitation of child sex offenders. Australian Psychologist, 37, 206-214.

Beech, A. R., \& Mann, R. (2002). Recent developments in the assessment and treatment of sexual offenders. In J. McGuire (Ed.), Offender rehabilitation and treatment: Effective programmes and policies to reduce re-offending (pp. 259-288). Chichester, UK: Wiley.

Blumenthal, S., Gudjonsson, G., \& Burns, J. (1999). Cognitive distortions and blame attribution in sex offenders against adults and children. Child Abuse \& Neglect, 23, $129-143$.

Briere, J. N. (1992). Child abuse trauma: theory and treatment of the lasting effects. Newbury Park, CA: Sage.

Browne, A. \& Finkelhor, D. (1986). Impact of child sexual abuse: A review of the research. Psychological Bulletin, 99, 66-77.

Bowens, B. (2004). Psychological defense mechanisms: A new perspective. The American Journal of Psychoanalysis, 64, 1-26. 
Bumby, K. M. (1996). Assessing the cognitive distortions of child molesters and rapists: Developments and validation of the MOLEST and RAPE scales. Sexual Abuse: A Journal of Research and Treatment, 8, 37-54.

Carpendale, J. I. \& Chandler, M. J. (1996). On the distinction between false belief understanding and subscribing to an interpretative theory of mind. Child Development, 67, 1686-1706.

Center for the Study of Emotion and Attention [CSEA-NIMH] (1999). The international affective picture system: Digitized photographs. Gainesville, FL: The Center for Research in Psychophysiology, University of Florida.

Chaffin, M. (1994). Research in action: Assessment and treatment of child sexual abusers. Journal of Interpersonal Violence, 9, 224-237.

Churchland, P. M. (1992). Eliminative materialism and the propositional attitudes. In P. M. Churchland (Ed.), A neurocomputational perspective: The Nature of Mind and the structure of science. (pp. 1-22). Cambridge, MA: MIT Press.

Clancey, W. J. (1993). Situated Action: A Neuropsychological Interpretation. Response to Vera and Simon. Cognitive Science, 17, 87 -116.

Clark, A. (1997). Being there: Putting brain, body and world together again. Cambridge, MA: MIT Press.

Collins, A. M., \& Loftus, E. F. (1975). A Spreading-Activation Theory of Semantic Processing. Psychological Review, 82, 407-428.

Copello, A. G., \& Tata, P. R. (1990). Violent behaviour and interpretive bias: An experimental study of the resolution of ambiguity in violent offenders. British Journal of Clinical Psychology, 2, 417-428.

Crowne, D. P., \& Marlowe, D. (1960). A new scale of social desirability independent of psychopathology. Journal of Consulting Psychology, 24, 349-354. 
Cumming, T. B., Graham, K. S., \& Patterson, K. (2006). Repetition priming and hyperpriming in semantic dementia. Brain and Language, 98, 221-234.

Curran, H. V., Rees, H., Hoare, T., Hoshi, R. \& Bond, A. (2004). Empathy and aggression: two faces of ecstasy? A study of interpretative cognitive bias and mood change in ecstasy users. Psychopharmacology, 173, 425-433.

Damasio, A. (1994). Descarte's error. London, UK: Penguin.

Denov, M. (2004). The long-term effects of child sexual abuse by female perpetrators: A qualitative study of male and female victims. Journal of Interpersonal Violence, $19,1137-1156$.

Dodge, K. A., \& Frame, C. L. (1982). Social cognitive biases and deficits in aggressive boys. Child Development, 33, 620-635.

Drake, C., Ward, T., Nathan, P., \& Lee, J. (2001). Challenging the cognitive distortions of child molesters: An implicit theory approach. Journal of Sexual Aggression, 7, $25-40$.

Drapeau, M., Korner, A. C., Granger, L., \& Brunet, L. (2005). What sex abusers say about their treatment: Results from a qualitative study on pedophiles in treatment at a Canadian penitentiary clinic. Journal of Child Sexual Abuse, 14, 91-115.

Duffy, S. A. (1986). Role of expectations in sentence integration. Journal of Experimental Psychology: Learning, Memory and Cognition, 12, 208-219.

Dweck, C. S., Chiu, C. \& Hong, Y. (1995). Implicit theories and their role in judgements and reactions: A world from two perspectives. Psychological Inquiry, 6, 267-285.

Fazio, R. H., \& Olson, M. A. (2003). Implicit measures in social cognition research: Their meaning and use. Annual Review of Psychology, 54, 297-328.

Feelgood, S., Cortini, F., \& Thompson, A. (2005). Sexual coping, general coping and cognitive distortions in incarcerated rapists and child molesters. Journal of Sexual Aggression, 11, 157-170. 
Fergusson, D.M., Horwood, L.J., \& Woodward, L.J. (2000). The stability of child abuse reports: a longitudinal study of the reporting behaviour of young adults. Psychological Medicine, 30, 529-544.

Fergusson, D. M., \& Mullen, P.E. (1999). Childhood sexual abuse: An evidence based perspective. Thousand Oaks, California: Sage Publications.

Fincher-Kiefer, R. (1992). The role of prior knowledge in inferential processing. Journal of Reading Research, 15, 12-27.

Fischler, I., \& Bloom, P. A. (1979). Automatic and attentional processes in the effects of sentence contexts on word recognition. Journal of Verbal Learning and Verbal Behaviour, 18, 1-20.

Fisher, D., Beech, A., \& Browne, K. (1999). Comparison of sex offenders to nonoffenders on selected psychological measures. International Journal of Offender Therapy and Comparative Criminology, 43, 473-491.

Fiske, S. T. \& Linville, P. W. (1980). What does a schema concept buy us? Personality and Social Psychology Bulletin, 6, 543-557.

Fiske, S. T. and Taylor, S. E. (1991). Social cognition (2nd ed.). New York: McGraw Hill.

Finkelhor (1984). Child sexual abuse: New theory and research. New York: The Free Press.

Fitzgerald, J. (1995). English-as-a-second-language learners' cognitive reading processes: A review of research in the United States. Review of Educational Research, 65, $145-190$.

Fliess, J. L. (1981). Statistical methods for rates and proportions. New York: John Wiley.

Forster, K. I. (1981). Priming and the effects of sentence and lexical contexts on naming time: Evidence for autonomous lexical processing. Quarterly Journal of Experimental Psychology, 33, 465-495. 
Freeman, A., Pretzer, J., Fleming, B., \& Simon, K. M. (2004). Clinical applications of cognitive therapy (2nd ed.). New York: Plenum Press.

Fromm, S. Total estimated cost of child abuse and neglect in the United States-statistical evidence. Chicago (IL): Prevent Child Abuse America (PCAA); 2001. [cited 2005 Jan 1]. Available from: URL:

www.preventchildabuse.org/learn_more/research_docs/cost_analysis.pdf.

Galambos, J. A., Abelson, R. P., \& Black, J. B. (1986). Knowledge structures. NJ, USA: Lawrence Erlbaum.

Gannon, T. A. (2006). Increasing Honest Responding on Cognitive Distortions in Child Molesters: The Bogus Pipeline Procedure. Journal of Interpersonal Violence, 21, $358-375$.

Gannon, T. A., Keown, K., \& Rose, M. R. (in press). An Examination of Current Psychometric Assessments of Child Molesters' Offense-Supportive Beliefs Using Ward's Implicit Theories. International Journal of Offender Therapy and Comparative Criminology.

Gannon, T. A., Keown, K., \& Polaschek, D. L. L. (2007). Increasing honest responding on cognitive distortions in child molesters: The bogus pipeline revisited. Sexual Abuse: A Journal of Research and Treatment, 19, 5-22.

Gannon, T. A., \& Polaschek, D. L. L. (2005). Do child molesters deliberately fake good on cognitive distortion questionnaires? An information processing-based investigation. Sexual Abuse: A Journal of Research and Treatment, 17, 183-200.

Gannon, T. A., \& Polaschek, D. L. L. (2006). Cognitive distortions in child molesters: A re-examination of key theories and research. Clinical Psychology Review, 26, 1000-1019. 
Gannon, T. A., Wright, D. B., Beech, A. R., \& Williams, S. (2006). Do child molesters hold distorted beliefs? What does their memory recall tell us? Journal of Sexual Aggression, 12, 5-18.

Geer, J. H., Estupinan, L. A., and Manguno-Mire, G. M. (1999). Empathy, social skills, and other relevant cognitive processes in rapists and child molesters. Aggression and Violent Behavior, 5, 99-126.

Gray, N. S., Brown, A. S., MacCulloch, M. J., Smith, J., \& Snowden, R. J. (2005). An implicit test of the association between children and sex in pedophiles. Journal of Abnormal Psychology, 114, 304-308.

Greenwald, A. G., \& Banaji, M. R. (1995). Implicit social cognition: Attitudes, selfesteem, and stereotypes. Psychological Review, 102, 4-27.

Greenwald, A. G., Banaji, M. R., Rudman, L. A., Farnham, S. D., Nosek, B. A., \& Mellott, D. S. (2002). A unified theory of implicit attitudes, stereotypes, selfesteem, and self-concept. Psychological Review, 109, 3-25.

Greenwald, A. G., \& Farnham, S. D. (2000). Using the Implicit Association Test to measure self-esteem and self-concept. Journal of Personality and Social Psychology, 79, 1022-1038.

Greenwald, A. G., McGhee, J. L., \& Schwartz, J. L. (1998). Measuring individual difference in implicit cognition: The Implicit Association Test. Journal of Personality and Social Psychology, 74, 1464-1480.

Gress, C. L. Z. (2005). Viewing time measures and sexual interest: Another piece of the puzzle. Journal of Sexual Aggression, 11, 117-125.

Groth, A. N., Hobson, W. F., \& Gary, T. S. (1982). The child molester: Clinical observations. In J. Conte \& D. A. Shore (Eds.), Social work and child sexual abuse (pp.129-144). New York: Haworth. 
Gudjonsson, G. H. (1984). Attribution of blame for criminality acts and its relationship with personality. Personality and Individual Differences, 5, 53-58.

Haidt, J. (2001). The emotional dog and its rational tail: A social intuitionist approach to moral judgment. Psychological Review, 108, 814-834.

Hall, G. C. N., \& Hirschman, R. (1991). Toward a theory of sexual aggression: A quadripartite model. Journal of Consulting and Clinical Psychology, 59, 662-669.

Hall, G. C. N., \& Hirschman, R. (1992). Sexual aggression against children. Criminal Justice and Behavior, 19, 8-23.

Hanson, R. K., Gizzarelli, R., \& Scott, H. (1994). The attitudes of incest offenders: Sexual entitlement and acceptance of sex with children. Criminal Justice and Behaviour, 21, 187-202.

Hanson, R. K., Gordon, A., Harris, A. J. R., Marques, J. K., Murphy, W., Quinsey, V. L. (2002). First report of the Collaborative Outcome Project on the effectiveness of psychological treatment for sex offenders. Sexual Abuse: A Journal of Research and Treatment, 14, 169- 194.

Hanson, R. K., \& Morton-Bourgon, K. (2004). Predictors of sexual recidivism: An updated meta-analysis. User report 2004-02 Ottawa: Public Safety and Emergency Preparedness Canada.

Harris, G. T., Rice, M. E., Quinsey, V. L., \& Chaplin, T. C. (1996). Viewing time as a measure of sexual interest among child molesters and normal heterosexual men. Behavioural Research and Therapy, 34, 389-394.

Hartley, C. C. (1998). How incest offenders overcome internal inhibitions through the use of cognitions and cognitive distortions. Journal of Interpersonal Violence, 13, 2539.

Hayashino, D. S., Wurtele, S. K., \& Klebe, K. J. (1995). Child molesters: An examination of cognitive factors. Journal of Interpersonal Violence, 10, 106-116. 
Hermann, J. L. (1990). Sex offenders: A feminist perspective. In W. L. Marshall, D. R. Laws, \& H. E. Barbaree (Eds.), Handbook of sexual assault: Issues, theories, and treatment of the offender (pp. 177-190). New York: Plenum Press.

Higgins, E. T. (1989). Knowledge accessibility and activation: Subjectivity and suffering from unconscious sources. In J. S. Uleman \& J. A. Bargh (Eds)., Unintended thought (pp. 75-123). New York: Guilford.

Holden, R.R., Kroner, D.G., Fekken, G.C., \& Popham, S.M. (1992). A model of personality test item response dissimulation, Journal of Personality and Social Psychology, 63, 272-279.

Hollon, S. D., \& Kriss, M. R. (1984). Cognitive factors in clinical research and practice. Clinical Psychology Review, 4, 35-76.

Howells, K., Day, A., \& Wright, S. (2004). Affect, emotions and sex offending. Psychology, Crime \& Law, 10, 179 - 195.

Howitt, D. (1995). Paedophiles and sexual offences against children. Chichester: Wiley.

Kamphuis, J H., De Ruiter, C., Janssen, B., \& Spiering, M. (2005). Preliminary Evidence for an Automatic Link Between Sex and Power Among Men Who Molest Children. Journal of Interpersonal Violence, 20, 1351-65

Kay, A. C., \& Jost, J. T. (2003). Complementary justice: Effects of "poor but happy" and "poor but honest" stereotype exemplars on system justification and implicit activation of the justice motive. Journal of Personality and Social Psychology, 85, 823-837.

Kihlstrom, J.F., \& Klein, S.B. (1994). The self as a knowledge structure. In R.S. Wyer \& T.K. Srull (Eds.), Handbook of social cognition, 2nd Ed. (Vol. 1, pp. 153-208). Hillsdale, N.J.: Erlbaum. 
Kirsch, L. G., \& Becker, J. V. (2006). Sexual offending: Theory of problem, theory of change, and implications for treatment effectiveness. Aggression and Violent Behavior, 11, 208-224.

Kolton, D. J. C., Boer, A., \& Boer, D. P. (2001). A revision of the Abel and Becker Cognition Scale for intellectually disabled offenders. Sexual Abuse: A Journal of Research and Treatment, 13, 217-219.

Kroner, D. G., \& Weekes, J. R. (1996). Balanced Inventory of Desirable Responding: Factor structure, reliability, and validity with an offender sample. Personality and Individual Differences, 21, 323-333.

Kunda, Z. (1999). Social cognition: Making sense of people (2nd ed.). Cambridge, UK: MIT Press.

Langevin, R. (1991). A note on the problem of response set in measuring cognitive distortions. Annals of Sex Research, 4, 287-292.

Lanyon, R. I. (1991). Theories of sexual offending. In C. R. Hollin, \& K. Howells (Eds.), Clinical approaches to sex offenders and their victims (pp. 35-54). New York: John Wiley.

Lanyon, R. I. (2001). Psychological assessment procedures in sex offending. Professional Psychology: Research and Practice, 32, 253-260.

Laws, D. R. \& Marshall, W. L. (1990). A conditioning theory of the etiology and maintenance of deviant sexual preference and behavior. In W. L. Marshall, D. R. Laws, \& H. E. Barbaree (Eds.), Handbook of sexual assault: Issues, theories, and treatment of the offender (pp. 209-229). New York: Plenum Press.

Lord, A., \& Willmot, P. (2007) The process of overcoming denial in sexual offenders. Journal of Sexual Aggression, 10, 51 - 61.

McFarlane-Nathan, G. (1999). FReMO - Framework for Reducing Māori Offending. Psychological Service, Department of Corrections, NZ. Available at: 
http://www.corrections.govt.nz/public/pdf/publications/fremo.pdf.

McGinn, L., \& Young, J. E. (1996). Schema-focused therapy. In P. M. Salkovskis (Ed.), Frontiers of cognitive therapy. (pp. 182-207). New York: Guilford Press.

McGrath, M., Cann, S., \& Konopasky, R. (1998). New measures of defensiveness, empathy, and cognitive distortions for sexual offenders against children. Sexual Abuse: A Journal of Research and Treatment, 10, 25-36.

Macleod, C. \& Cohen, I. L. (1993). Anxiety and the interpretation of ambiguity: A text comprehension study. (1993). Journal of Abnormal Psychology, 102, 238-247.

Malamuth, N. M., \& Brown, L. M. (1994). Sexually aggressive men's perceptions of women's communications: Testing three explanations. Journal of Personality and Social Psychology, 67, 699-712.

Malamuth, N. M., Heavey, C.L. \& Linz, D. (1993). Predicting men's antisocial behavior against women: The interaction model of sexual aggression. In G. C. Hall, R. Hirschman, J. R. Graham, \& M. S. Zaragoza (Eds.), Sexual Aggression (Issues in Etiology, Assessment, and Treatment) (pp. 63 -99). Philadelphia, US: Taylor \& Francis.

Malle, B. F. (2006). How the mind explains behavior: Folk explanations, meaning, and social interaction. Cambridge, UK: MIT Press.

Mann, R. E., \& Beech, A. R. (2003). Cognitive distortions, schemas, and implicit theories. In T. Ward, D. R. Laws, \& S. M. Hudson (Eds.)., Sexual deviance: Issues and controversies (pp. 135-153), Thousand Oaks, CA: Sage.

Mann, R. E., \& Hollin, C. R. (2007). Sexual offenders' explanations for their offending. Journal of Sexual Aggression, 13, 3-9.

Mann, R. E., Webster, S., Wakeling, H., \& Marshall, W. (2007). The measurement and influence of child sexual abuse supportive beliefs. Psychology, Crime \& Law, 13, $443-458$. 
Marques, J. K., Wiederanders, M., Day, D. M., Nelson, C., \& van Ommeren, A. (2005). Effects of a relapse prevention program on sexual recidivism: Final results from California's Sex Offender Treatment and Evaluation Project (SOTEP). Sexual Abuse: A Journal of Research and Treatment, 17, 79-107.

Marshall, W. L. (1999). Current status of North American assessment and treatment programs for sexual offenders. Journal of Interpersonal Violence, 14, 221-239.

Marshall, W. L., Anderson, D., \& Fernandez, Y. (1999b). Cognitive behavioural treatment of sexual offenders, Toronto: Wiley.

Marshall, W. L., \& Barbaree, H. E. (1990). Outcome of comprehensive cognitivebehavioural treatment programmes. In W. L. Marshall, D. R. Laws, \& H. E. Barbaree (Eds.), Handbook of sexual assault: Issues, theories, and treatment of the offender (pp. 363-385). New York: Plenum Press.

Marshall, W. L. \& Barbaree, H. E. (1999). Cognitive Behavioural Treatment of Sexual Offenders. Chichester, UK: John Wiley.

Marshall, W. L., Cripps, E., Anderson, D. \& Cortoni, F. A. (1999a). Self-esteem and coping strategies in child molesters. Journal of Interpersonal Violence, 14, 955962.

Marshall, W. L., Marshall, L. E., Sachdav, S., \& Kruger, R. (2003). Distorted attitudes and perceptions, and their relationship with self esteem and coping in child molesters. Sexual Abuse: A Journal of Research and Treatment, 15, 171-181.

Maruna, S., \& Mann, R. E. (2006). A fundamental attribution error?: Rethinking cognitive distortions. Legal and Criminological Psychology, 11, 155-177.

Marziano, V. (2002). Male child molesters' implicit theories, Unpublished DPsych thesis, University of Melbourne, Melbourne, Victoria. 
Marziano, V., Ward, T., Beech, A. R., Pattison, P. (2006). Identification of five fundamental implicit theories underlying cognitive distortions in child abusers: A preliminary study. Psychology, Crime \& Law, 12, 97-105.

Meyer, D.E., \& Schvaneveldt, R.W. (1971). Facilitation in recognizing pairs of words: Evidence of a dependence between retrieval operations. Journal of Experimental Psychology, 90, 227-234.

Mihailides, S., Devilly, G. J., \& Ward, T. (2004). Implicit cognitive distortions and sexual offending. Sexual Abuse: A Journal of Research and Treatment, 16, 333-350.

Mikulincer, M. (1998). Adult attachment style and individual differences in functional versus dysfunctional experiences of anger. Journal of Personality and Social Psychology, 74, 513-524.

Mikulincer, M., Birnbaum, G., Woddis, D., \& Nachmias, O. (2000). Stress and proximity of proximity-related thoughts: Exploring the normative and intraindividual components of attachment theory. Journal of Personality and Social Psychology, 78, 509-523.

Milgram, S. (1963). Behavioural study of obedience. Journal of Abnormal and Social Psychology, 67, 371-378.

Milner, R. J., \& Webster, S. D. (2005). Identifying schemas in child molesters, rapists, and violent offenders. Sexual Abuse: A Journal of Research and Treatment, 17, 425439.

Mullen, P. E., Martin, J. L., Anderson, J. C., Romans, S. E. \& Herbison, G. P. (1996). The long-term impact of the physical, emotional, and sexual abuse of children: A community study. Child Abuse and Neglect, 20, 7-21.

Murphy, W. D. (1990). Assessment and modification of cognitive distortions in sex offenders. In W. L. Marshall, D. R. Laws, \& H. E. Barbaree (Eds.)., Handbook of 
sexual assault: Issues, theories, and treatment of the offender (pp. 331-342), New York: Plenum.

Murray, J. D., Spadafore, J. A., \& McIntosh, W. D. (2005). Belief in a just world and social perception: Evidence for automatic activation. Journal of Social Psychology, $145,35-47$.

Neely, J. H. (1991). Semantic priming effects in visual word recognition: A selective review of current findings and theories. In Besner, D., \& Humphreys, G. W. (Eds.)., Basic Processes in Reading: Visual Word Recognition (pp 264-336), Hillsdale, NJ: Lawrence Erlbaum.

Neidigh, L., \& Krop, H. (1992). Cognitive distortions among child sexual offenders. Journal of Sex Education and Therapy, 18, 208-215.

Nisbett, R. E., \& Ross, L. (1980). Human inference: Strategies and shortcomings of social judgment. Englewood Cliffs, NJ: Prentice-Hall.

Nisbett, R. E., \& Wilson, T. D. (1977). Telling more than we can know: Verbal reports on mental processes. Psychological Review, 84, 231-259.

Nunes, K. L., Firestone, P., \& Baldwin, M. A. (2007). Indirect assessment of cognitions of child sexual abusers with the Implicit Association Test. Criminal Justice and Behavior, 34, 454-475.

Pacific Psychological Assessment Corporation (PPAC). (2004). The NRP (Not Real People) stimulus set for assessment of sexual interest. Victoria, BC, Canada: Author.

Palmer, E. (2003). An overview of the relationship between moral reasoning and offending. Australian Psychologist, 38, 165-174.

Palmer, E.J. (2000). Perceptions of parenting, social cognition and delinquency. Clinical Psychology and Psychotherapy, 7, 303-309. 
Paulhus, D. L. (1984). Two-component models of socially desirable responding. Journal of Personality and Social Psychology, 46, 598-609.

Paulhus, D. L. (1988). Assessing self-deception and impression management in selfreports: The Balanced Inventory of Desirable Responding. Unpublished Manual, University of British Columbia, Vancouver, Canada.

Pecher, D. \& Zwaan, R. (Eds.). (2005). Grounding cognition: The role of perception and action in memory, language and thinking. Cambridge, UK: Cambridge University Press.

Pervan, S., \& Hunter, M. (2007). Cognitive distortions and social self-esteem in sexual offenders. Applied Psychology in Criminal Justice, 3, 75-91.

Phenix, A., Hanson, R. K., \& Thornton, D. (2000). Coding rules for the Static-99. Corrections Research: Manuals and Forms. Ottawa: Department of the Solicitor General of Canada.

Pollock, N. L., \& Hashmall, J. M. (1991). The excuses of child molesters. Behavioral Sciences \& the Law, 9, $53-59$.

Pryor, J. B., \& Stoller, L. M. (1994). Sexual cognition processes in men high in the likelihood to sexually harass. Personality and Social Psychology Bulletin, 2, 163169.

Purvis, M., \& Joyce, A. (2005). Child sexual abuse is a global public health problem: Where is Australia? Psychiatry, Psychology, and Law, 12, 334-344.

Quinsey, V. L. (1998). Comment on Marshall's "Monster, Victim, or Everyman". Sexual Abuse: A Journal of Research and Treatment, 10, 65-69.

Ratcliff R. (1993). Methods for dealing with reaction time outliers. Psychological Bulletin, 114, 510-532.

Rogers, R. \& Dickey, R. (1991). Denial and minimization among sex offenders: A review of competing models of deception. Annals of Sex Research, 4, 49-63. 
Rudman, Greenwald, A. G., Mellot, D. S., \& Schwartz, J L. K. (1999). Measuring the automatic components of prejudice: Flexibility and generality of the implicit association test. Social Cognition, 17, 437-466.

Rumelhart, D. E., McClelland, J. L., and the PDP Research Group (1986). Parallel distributed processing: Explorations in the microstructure of cognition, vol. 1: Foundations. Cambridge, MA: MIT Press.

Saradjian, A., \& Nobus, D. (2003). Cognitive distortions of religious professionals who abuse children. Journal of Interpersonal Violence, 18, 905-923.

Schank, R.C. \& Abelson, R. (1977). Scripts, plans, goals, and understanding. Hillsdale, NJ: Earlbaum Associates.

Schroder, H. M., Driver, M. J., \& Streufert, S. (1967). Human information processing. New York: Holt, Rinehart and Winston.

Segal, Z. V., \& Stermac, L. E. (1990). The role of cognition in sexual assault. In W. L. Marshall, D. R. Laws, \& H. E. Barbaree (Eds.), Handbook of sexual assault: Issues, theories, and treatment of the offender (pp. 161-174). New York: Plenum Press.

Sharkey, N., \& Sharkey, A. (1987). What is the point of facilitation? The loci of knowledge-based facilitation in sentence processing. Journal of Memory and Language, 26, 255-276.

Sherman, S. J., Judd, C. M., \& Park, B. (1989). Social cognition. Annual Review of Psychology, 40, 281-326.

Sinclair, G. P., Healy, A. F., Bourne, L. E. (1989). Facilitating text memory with additional processing opportunities in rapid sequential reading. Journal of Experimental Psychology: Learning, Memory, and Cognition, 15, 418-431.

Soederberg-Miller, L. M. (2001). The effects of real-world knowledge on text processing among older adults. Aging, Neuropsychology, \& Cognition, 8, 137-148. 
Stermac, L., \& Segal, Z. (1989). Adult sexual contact with children: An examination of cognitive factors. Behavior Therapy, 20, 573-584.

Stermac, L. E., Segal, Z. V., \& Gillis, R. (1990). Social and cultural factors in sexual assault. In W. L. Marshall, D. R. Laws, \& H. E. Barbaree (Eds.), Handbook of sexual assault: Issues, theories, and treatment of the offender (pp. 143-159). New York: Plenum Press.

Swanson, J. E., Rudman, L. A., \& Greenwald, A. G. (2001). Using the Implicit Association Test to investigate attitude-behaviour consistency for stigmatised behaviour. Cognition and Emotion, 15, 207-230.

Szentagotai, A., Schnur, J., DiGiuseppe, R. Macavei, B., Kallay, E., \& David, D. (2005). The organization and the nature of irrational beliefs: Schemas or appraisal? Journal of Cognitive and Behavioral Psychotherapies: Special Issue, 5, 139-158.

Thagard, P. (2006). Hot thought: Mechanisms and applications of emotional cognition. Cambridge, MA: MIT Press.

Tierney, D. W., \& McCabe, M. P. (2001). An evaluation of self-report measures of cognitive distortions and empathy among Australian sex offenders. Archives of Sexual Behaviour, 30, 495-519.

Vanhouche, W., \& Vertommen, H. (1999). Assessing cognitive distortions in sex offenders: A review of commonly used versus recently developed instruments. Psychologica Belgica, 39, 163-187.

Varela, F. J., Thompson, E. \& Rosch, E. (1991). The embodied mind: Cognitive science and human experience. Cambridge, US: MIT Press.

Vivian-Byrne, S. (2004). Changing people's minds. Journal of Sexual Aggression, 10, $181-192$.

Walters, G. D. (1995). The Psychological Inventory of Criminal Thinking Styles: I. Reliability and preliminary validity. Criminal Justice and Behavior, 22, 307-325. 
Walters, G. D. (2002). Criminal Belief Systems: An Integrated-Interactive Theory of Lifestyles. Westport, CT: Praeger Publishers.

Ward, T. (2000). Sexual offenders' cognitive distortions as implicit theories. Aggression and Violent Behavior: A Review Journal, 5, 491-507.

Ward, T., \& Beech, A. R., (2006). An integrated theory of sexual offending. Aggression and Violent Behavior, 11, 44-63.

Ward, T., Fon, C., Hudson, S. M., \& McCormack, J. (1998). A descriptive model of dysfunctional cognitions in child molesters. Journal of Interpersonal Violence, 13, 129-155.

Ward, T., \& Hudson, S. M. (1998). The construction and development of theory in the sexual offending area: A metatheoretical framework. Sexual Abuse: A Journal of Research and Treatment, 10, 47-63.

Ward, T., Hudson, S. M., Johnston, L., \& Marshall, W. L. (1997). Cognitive distortions in sex offenders: An integrative review. Clinical Psychology Review, 17, 1-29.

Ward, T., Hudson, S. M., \& Marshall, W. L. (1995). Cognitive distortions and affective deficits in sex offenders: A cognitive deconstructionist interpretation. Sexual Abuse: A Journal of Research and Treatment, 7, 67-83.

Ward, T., Hudson, S. M., Marshall, W. L., \& Siegert, R. J. (1995). Attachment style and interpersonal deficits in sexual offenders. Sexual Abuse: A Journal of Research and Treatment, 33, 17-26.

Ward, T. \& Keenan, T. (1999). Child molesters' implicit theories. Journal of Interpersonal Violence, 14, 821-838.

Ward, T., Keown, K., \& Gannon, T. A. (2007). Child sexual abuse-related cognition: Current research. In Gannon, T. A., Ward, T., \& Beech, A. R., \& Fisher, D. (Eds). Aggressive offenders' cognition: Theory, research and practice. Chichester, UK: John Wiley. 
Ward, T., Louden, K., Hudson, S. M., \& Marshall, W. L. (1995). A descriptive model of the offense chain for child molesters. Journal of Interpersonal Violence, 10, 452472.

Ward, T., \& Marshall, W. L. (2007). Narrative identity and offender rehabilitation. International Journal of Offender Therapy and Comparative Criminology, 51, $279-297$.

Ward, T. \& Nee, C. (in press). Surfaces and Depths: Evaluating the Theoretical Assumptions of Cognitive Skills Programmes. Psychology, Crime \& Law.

Ward, T., \& Siegert, R. J. (2002). Toward a comprehensive theory of child sexual abuse: A theory knitting perspective. Psychology, Crime and Law, 8, 319-351.

Welford, A. T. 1980. Choice reaction time: Basic concepts. In A. T. Welford (Ed.), Reaction times (pp. 73-128), NY: Academic Press.

Wilson, M. (2002). Six views of embodied cognition. Psychonomic Bulletin \& Review, 9, $625-636$.

Wingrove, J. \& Bond, A. J. (2005). Correlation between trait hostility and faster reading times for sentences describing angry reactions to ambiguous situations. Cognition \& Emotion, 19, $463-472$.

Wright, R. C., Schneider, S. L. (1999). Motivated self-deception in child molesters. Journal of Child Sexual Abuse, 8, 89-111. 


\section{Appendix One}

Study One: Simple and Complex Syntactic Sentences Used for Reading Speed Assessment (taken from Fischler \& Bloom, 1979)

\begin{tabular}{lcc}
\hline Simple Sentences & $\begin{array}{c}\text { Flesch } \\
\text { Score }\end{array}$ & $\begin{array}{c}\text { Number of } \\
\text { Words }\end{array}$ \\
\hline The hungry bear found some stale bread. & 94 & 8 \\
The death of his dog was a great shock. & 100 & 9 \\
Billy hit his sister on the head. & 90.9 & 7 \\
Jim had learned the special passage by heart. & 92.9 & 8 \\
They rested under a tree in the shade. & 92.9 & 8 \\
He mailed the letter without a stamp. & 90.9 & 7 \\
He bought them in a candy store. & 100 & 7 \\
He drove the nail into the wood. & 100 & 7 \\
Plants will not grow in dry soil. & 100 & 7 \\
She called her husband at his office. & 90.9 & 7 \\
Complex sentences & & \\
Three people were killed in a major highway accident. & 66.1 & 9 \\
The judge warned about the dangers of drinking. & 82.3 & 8 \\
The person who caught the thief deserves our thanks. & 94.3 & 9 \\
The surgeon tried vainly to save his patient. & 71.8 & 8 \\
The car stalled because the engine failed to start. & 94.3 & 9 \\
Rushing out he forgot to take his coat. & 92.9 & 8 \\
The police had never seen a man so drunk. & 94.3 & 9 \\
The surface of the water was nice and smooth. & 94.3 & 9 \\
Few had the nerve to take the needed shot. & 100 & 9 \\
George could not believe his son stole the car. & 94.3 & 9 \\
\hline & & \\
\hline
\end{tabular}




\section{Appendix Two}

Study One: LDT Context Sentences and Target Words by Implicit Theory

\begin{tabular}{|c|c|c|c|c|}
\hline \multirow[t]{2}{*}{ Implicit Theory } & \multirow[t]{2}{*}{ Word Stem } & \multicolumn{3}{|c|}{ Target Word Type } \\
\hline & & $\begin{array}{l}\text { Offence } \\
\text { Supportive }\end{array}$ & $\begin{array}{c}\text { Not } \\
\text { Offence } \\
\text { Supportive }\end{array}$ & Nonword \\
\hline \multirow[t]{8}{*}{$\begin{array}{l}\text { Dangerous } \\
\text { World }\end{array}$} & $\begin{array}{l}\text { On the whole, people } \\
\text { are }\end{array}$ & mean & nice & vull \\
\hline & $\begin{array}{l}\text { Most of us are really } \\
\text { seeking }\end{array}$ & power & love & umile \\
\hline & The world is full of & danger & people & tipsoe \\
\hline & $\begin{array}{l}\text { Most people want to do } \\
\text { People who trust others }\end{array}$ & $\begin{array}{l}\text { damage } \\
\text { strange }\end{array}$ & $\begin{array}{c}\text { good } \\
\text { normal }\end{array}$ & $\begin{array}{l}\text { hinger } \\
\text { snither }\end{array}$ \\
\hline & $\begin{array}{l}\text { are } \\
\text { People look out for }\end{array}$ & themselves & others & ungerstand \\
\hline & $\begin{array}{l}\text { If you don't strike first } \\
\text { you are }\end{array}$ & weak & slow & brab \\
\hline & $\begin{array}{l}\text { Compared to adults, } \\
\text { children are }\end{array}$ & safe & small & salk \\
\hline & $\begin{array}{l}\text { The best people to trust } \\
\text { are }\end{array}$ & kids & adults & Devy \\
\hline \multirow[t]{9}{*}{ Entitlement } & $\begin{array}{l}\text { When you're lonely, } \\
\text { turning to a child for } \\
\text { sex is }\end{array}$ & fine & bad & lish \\
\hline & $\begin{array}{l}\text { When it comes to sex, } \\
\text { sometimes a man must } \\
\text { exercise his }\end{array}$ & rights & will & meadle \\
\hline & $\begin{array}{l}\text { A man who puts his } \\
\text { needs ahead of his } \\
\text { child's is }\end{array}$ & strong & mean & sheeze \\
\hline & $\begin{array}{l}\text { The idea that men are } \\
\text { better than women or } \\
\text { children is }\end{array}$ & right & false & lauth \\
\hline & $\begin{array}{l}\text { If a man is deprived of } \\
\text { sex, sleeping with a } \\
\text { child is }\end{array}$ & okay & wrong & zine \\
\hline & $\begin{array}{l}\text { Doing what I want, } \\
\text { when I want is }\end{array}$ & good & wrong & zine \\
\hline & $\begin{array}{l}\text { Always putting yourself } \\
\text { first is }\end{array}$ & healthy & unfair & ottager \\
\hline & $\begin{array}{l}\text { If I hurt others to get } \\
\text { what I need, I feel }\end{array}$ & fine & guilty & kilp \\
\hline & $\begin{array}{l}\text { If a woman doesn't give } \\
\text { a man what he needs, } \\
\text { he must exercise }\end{array}$ & force & control & droam \\
\hline
\end{tabular}




\begin{tabular}{|c|c|c|c|c|}
\hline \multirow[t]{2}{*}{ Implicit Theory } & \multirow[t]{2}{*}{ Word Stem } & \multicolumn{3}{|c|}{ Target Word Type } \\
\hline & & $\begin{array}{l}\text { Offence } \\
\text { Supportive }\end{array}$ & $\begin{array}{c}\text { Not } \\
\text { Offence } \\
\text { Supportive }\end{array}$ & Nonword \\
\hline \multirow[t]{9}{*}{ Uncontrollability } & $\begin{array}{l}\text { Sexual relations with } \\
\text { children cannot be }\end{array}$ & helped & allowed & mollow \\
\hline & $\begin{array}{l}\text { Sometimes, giving in to } \\
\text { a sexual urge is a }\end{array}$ & must & crime & reak \\
\hline & $\begin{array}{l}\text { When a man says he } \\
\text { can't resist touching } \\
\text { children he is }\end{array}$ & right & wrong & caich \\
\hline & $\begin{array}{l}\text { Sex with some children } \\
\text { is simply }\end{array}$ & inevitable & ignored & steaighten \\
\hline & People can't control & anything & everything & jolidify \\
\hline & $\begin{array}{l}\text { People who try to } \\
\text { change things are }\end{array}$ & odd & great & nop \\
\hline & $\begin{array}{l}\text { Being unable to resist } \\
\text { temptation is }\end{array}$ & normal & bad & scleam \\
\hline & $\begin{array}{l}\text { People who try to make } \\
\text { changes in their lives } \\
\text { are }\end{array}$ & blind & healthy & skretch \\
\hline & $\begin{array}{l}\text { Giving in to all your } \\
\text { sexual urges is just }\end{array}$ & human & weak & drike \\
\hline \multirow[t]{9}{*}{ Nature of Harm } & $\begin{array}{l}\text { Just touching a child's } \\
\text { private parts is }\end{array}$ & okay & wrong & gwin \\
\hline & $\begin{array}{l}\text { The belief that sex } \\
\text { harms all children is }\end{array}$ & false & true & falm \\
\hline & $\begin{array}{l}\text { Having sex with } \\
\text { children won't do them } \\
\text { any }\end{array}$ & harm & good & knid \\
\hline & $\begin{array}{l}\text { If a child is quiet during } \\
\text { sexual activity, they are } \\
\text { probably feeling }\end{array}$ & glad & shocked & eddress \\
\hline & $\begin{array}{l}\text { Sexual activity can } \\
\text { contribute to a child's }\end{array}$ & learning & problems & struddle \\
\hline & $\begin{array}{l}\text { A child who is fondled } \\
\text { by an adult will } \\
\text { probably be }\end{array}$ & okay & hurt & jick \\
\hline & $\begin{array}{l}\text { If a child is molested } \\
\text { while they sleep they } \\
\text { will probably be }\end{array}$ & fine & hurt & jerf \\
\hline & $\begin{array}{l}\text { Some children don't tell } \\
\text { if they have been } \\
\text { fondled because they } \\
\text { are secretly }\end{array}$ & pleased & frightened & kribble \\
\hline & $\begin{array}{l}\text { Sexual activity with a } \\
\text { child can teach that } \\
\text { child to }\end{array}$ & love & fear & prink \\
\hline
\end{tabular}




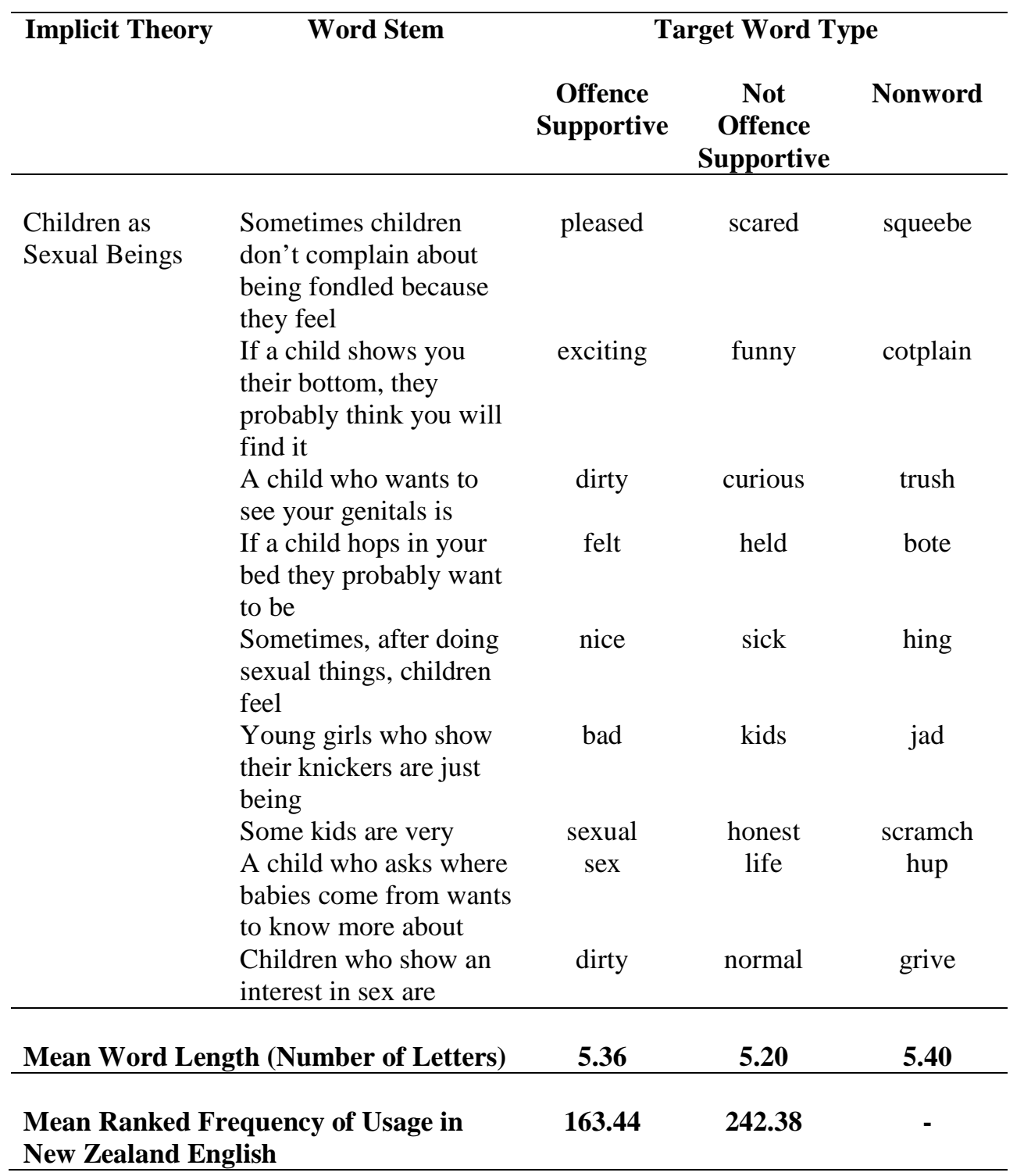




\section{Appendix Three}

Study Two: Examples of Child Priming Pictures
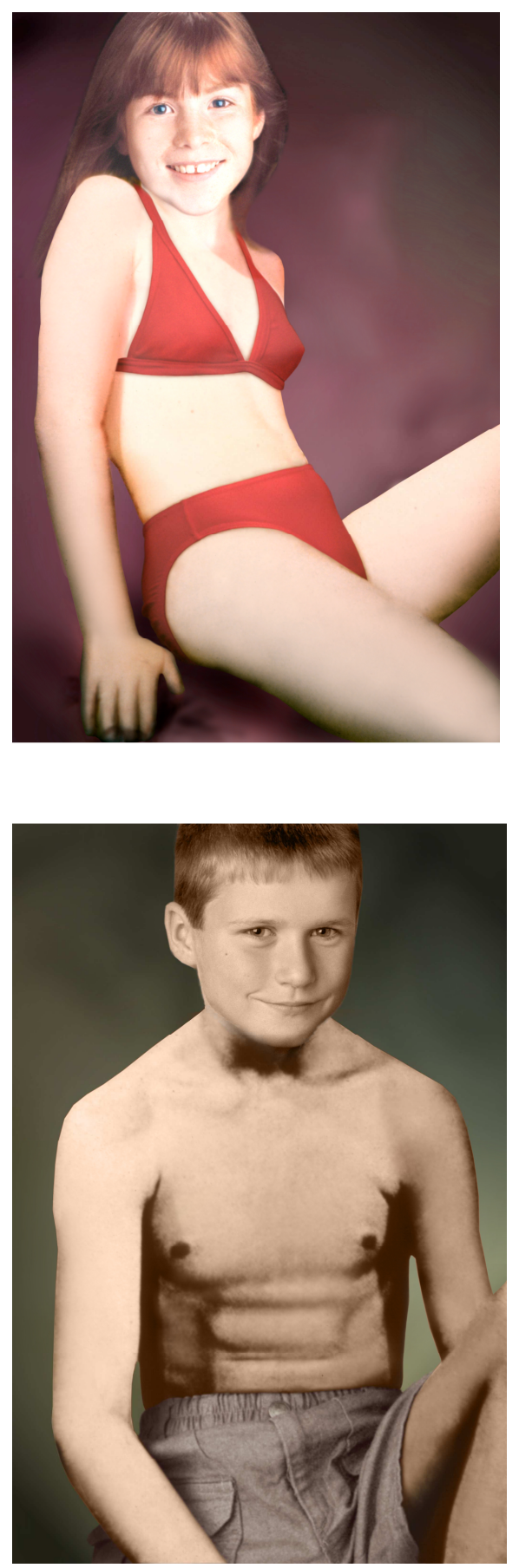


\section{Appendix Four}

Study Two: Examples of Adult Priming Pictures
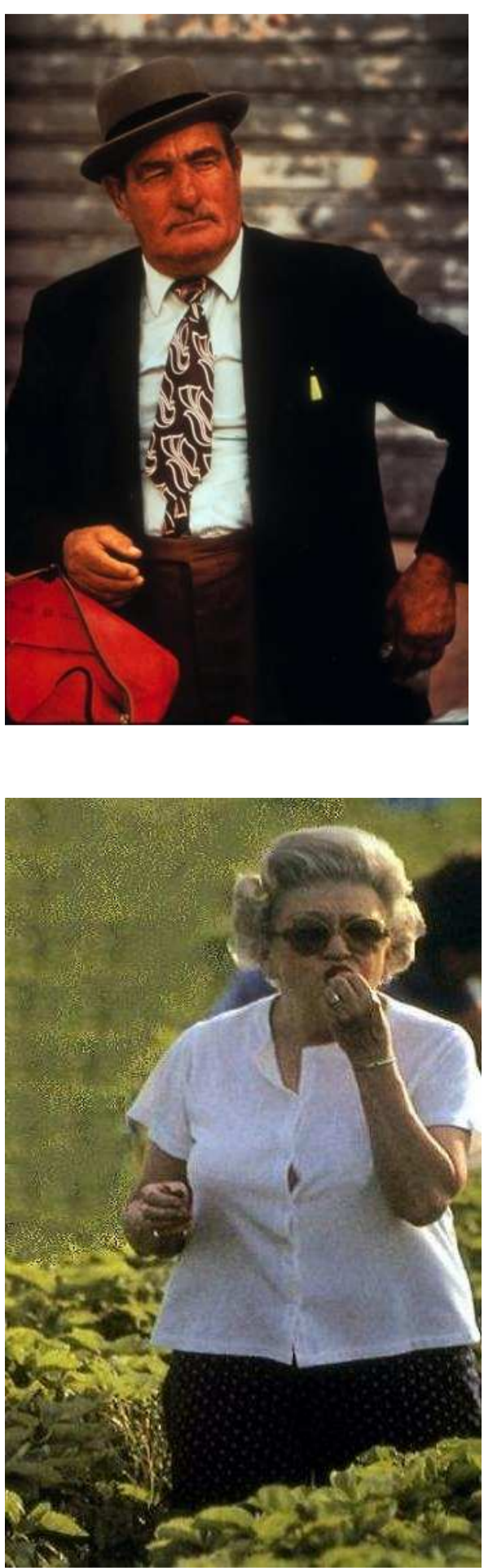


\section{Appendix Five}

\section{Study Two: Encoding Sentences}

Sentence Type and Number

Sexual 1 As 7-year old Jenny played cards, she showed her knickers.

Sexual 2 As Dave fondled the little boy, the boy let out a whimper.

Sexual 3 Jane didn't tell anyone about her baby sitter's "touches".

Sexual $4 \quad$ The coach told 10-year old Jo to stop teasing him.

Sexual 5 During the film, Ken felt his young niece looking at him.

Sexual $6 \quad$ At the school gates, Kim kissed her teacher goodbye.

Sexual $7 \quad$ Kate told her Uncle that she needed help to read the sexual education book.

Sexual $8 \quad$ Scott didn't say anything as the priest undressed him.

Sexual $9 \quad$ Young Beth kept wriggling about on the man's knee.

Sexual $10 \quad$ The 13-year old climbed into bed with the farm-worker

Threatening 1 The doctor frowned as he measured little Sarah's growth.

Threatening 2 Rob's behaviour made it hard for Amy to hide her feelings towards him.

Threatening 3 Joseph's exhibition attracted a lot of attention.

Threatening 4 After several hours, they decided that the leg was beyond repair.

Threatening 5 All the guests at the wedding giggled at Mark's speech.

Threatening 6 The farmer turned to Dave and gave him the sack.

Threatening $7 \quad$ Lisa was surprised when she saw her exam results.

Threatening 8 The party Steve threw turned out just as he had expected it to.

Threatening 9 When the mountaineers returned from the top, Mike was let down by Alan.

Threatening 10 Before leaving, the couple wanted to speak to the chef about their meal.

Neutral Filler 1 The pharmacist told the old lady to take her medicine every morning.

Neutral Filler 2 The two old friends played pool until closing time.

Neutral Filler 3 The teacher started the lesson at the school.

Neutral Filler 4 She was very surprised to receive the birthday card. 


\section{Study Two: Encoding Sentences (Continued)}

Sentence Type and Number

\section{Sentence Text}

Neutral Filler 5 The man started his morning exercises.

Neutral Filler 6 The secretary was given a pay rise at the end of her first year in the company.

Neutral Filler 7 It took the man all day to paint the kitchen.

Neutral Filler 8 After having waited for the bus for half an hour the man decided to walk home.

Neutral Filler 9 The landlord arrived at the door where the dog was barking.

Neutral Filler 10 The man drove the old car to the garage to get the oil filter changed. 


\section{Appendix Six}

Study Two: Recognition Sentences

Sentence Type and Number

\section{Sentence Text}

Sexual $1 \quad$ While Jenny played cards, she deliberately showed her knickers.

Nonsexual 1 While Jenny played cards, she accidentally showed her knickers.

Sexual 2 As Dave fondled the little boy, the boy let out a happy whimper.

Nonsexual 2 As Dave fondled the little boy, the boy let out a shocked whimper.

Sexual $3 \quad$ Jane didn't mind her babysitter's secret touches.

Nonsexual 3 Jane worried about her babysitter's secret touches.

Sexual $4 \quad$ The coach told 10-year old Jo to stop flirting with him.

Nonsexual 4 The coach told 10-year old Jo to stop being cheeky with him.

Sexual $5 \quad$ During the film, Ken thought his niece was being seductive.

Nonsexual $5 \quad$ During the film, Ken thought his niece was looking bored.

Sexual 6 At the school gates, Kim showed she was attracted to her teacher.

Nonsexual 6 At the school gates, Kim showed she was going to miss her teacher.

Sexual $7 \quad$ Kate told her Uncle that she wanted a sexual education lesson.

Nonsexual $7 \quad$ Kate told her Uncle that she wanted a reading lesson.

Sexual $8 \quad$ Scott didn't want to stop the priest undressing him.

Nonsexual $8 \quad$ Scott didn't know how to stop the priest undressing him.

Sexual $9 \quad$ Young Beth moved suggestively about on the man's knee.

Nonsexual 9 Young Beth moved uncomfortably about on the man's knee.

Sexual $10 \quad$ The naughty 13-year-old climbed into the farm-worker's bed.

Nonsexual 10 The obedient 13-year-old climbed into the farm-worker's bed.

Threatening 1 The doctor frowned as he measured little Sarah's cancer.

Nonthreatening 1 The doctor frowned as he measured little Sarah's height.

Threatening 2 Rob's behaviour made it hard for Amy to hide her dislike for him.

Nonthreatening 2 Rob's behaviour made it hard for Amy to hide her affection for him.

Threatening 3 The havoc Joseph created grabbed people's attention.

Nonthreatening 3 The art Joseph created grabbed people's attention.

Threatening $4 \quad$ After several hours the doctor decided that the leg was beyond repair.

Nonthreatening 4 After several hours the carpenter decided that the leg was beyond repair.

Threatening 5 All the guests at the wedding ridiculed Mark's speech.

Nonthreatening 5 All the guests at the wedding enjoyed Mark's speech. 
Study Two: Recognition Sentences (Continued)

Sentence Type

and Number

Sentence Text

Threatening 6 The farmer turned to Dave and gave him his notice.

Nonthreatening 6 The farmer turned to Dave and gave him the bag.

Threatening $7 \quad$ Lisa was surprised that her results were so bad.

Nonthreatening 7 Lisa was surprised that her results were so good.

Threatening 8 The party Steve threw turned out to be a complete disaster.

Nonthreatening 8 The party Steve threw turned out to be a complete success.

Threatening 9 When the mountaineers returned from the top, Mike was disappointed by Alan.

Nonthreatening 9 When the mountaineers returned from the top, Mike was lowered down by Alan.

Threatening 10 Before leaving, the couple wanted to complain to the chef about their meal.

Nonthreatening 10 Before leaving, the couple wanted to complement the chef about their meal.

Neutral Filler 1 The pharmacist prescribed the medicine to the old lady.

Neutral Filler 2 The two friends played pool until the pub closed.

Neutral Filler 3 The lesson was started by the teacher at the school.

Neutral Filler 4 She was amazed when she received the birthday card.

Neutral Filler 5 In the morning, as usual the man started exercising.

Neutral Filler 6 The secretary had a salary increase after working in the firm for one year.

Neutral Filler 7 The man spent all day painting the kitchen.

Neutral Filler 8 He decided to walk home after waiting for the bus for too long.

Neutral Filler 9 The dog was barking when the landlord arrived at the door.

Neutral Filler 10 The man went to the garage so that the oil filter could be changed. 


\section{Appendix Seven}

\section{Study Three: CSO Interview Schedule}

1. Can you please begin to walk me through the first time that you had sexual contact with a child? What happened that lead up to that first sexual contact?

2. When did you first start thinking about the child in a sexual way?

3. And what actually happened during the first sexual contact with that child?

4. What were you experiencing at the time? How were you feeling?

5. What kind of thoughts were going through your mind about yourself, your life, your future?

6. During the time that you were offending, what kinds of things did you say to yourself that made it easier to go ahead and do the offending?

7. What was it about this particular child that made you want to have sexual contact with him/her?

8. How did the victim react to what happened?

9. Looking back at it, how do you make sense of your offending?

10. How did you feel about what had happened following the offence?

11. What did you say to yourself afterwards?

Repeat questions for offenders' other victims.

Finish by summarising interview and reiterating key points raised, then thank for sharing the information. 


\section{Appendix Eight}

Study Three: Participant Questionnaire 
ERROR: undefined

OFFENDING COMMAND: deN

STACK:

758

/pp_by2 